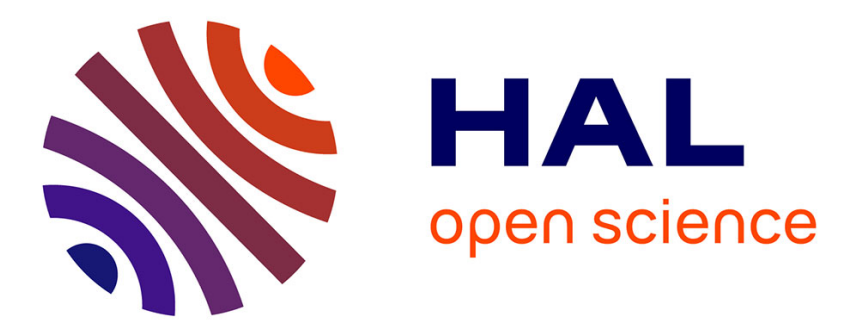

\title{
Liganded silver and gold quantum clusters. Towards a new class of nonlinear optical nanomaterials
}

\author{
Rodolphe Antoine, Vlasta Bonačić- Koutecký
}

\section{To cite this version:}

Rodolphe Antoine, Vlasta Bonačić- Koutecký. Liganded silver and gold quantum clusters. Towards a new class of nonlinear optical nanomaterials. Springer, Cham, 2018, 978-3-319-64742-5. 10.1007/9783-319-64743-2 . hal-02980213

\section{HAL Id: hal-02980213 \\ https://hal.science/hal-02980213}

Submitted on 1 Nov 2020

HAL is a multi-disciplinary open access archive for the deposit and dissemination of scientific research documents, whether they are published or not. The documents may come from teaching and research institutions in France or abroad, or from public or private research centers.
L'archive ouverte pluridisciplinaire HAL, est destinée au dépôt et à la diffusion de documents scientifiques de niveau recherche, publiés ou non, émanant des établissements d'enseignement et de recherche français ou étrangers, des laboratoires publics ou privés. 


\section{Springer Series : SpringerBriefs in Materials}

\section{Liganded silver and gold quantum clusters.}

Towards a new class of nonlinear optical nanomaterials

\section{Rodolphe Antoine}

Institut Lumière matière, UMR5306 - UCBL - CNRS, 10 rue Ada Byron, 69622 Villeurbanne cedex, France, rodolphe.antoine@univ-lyon1.fr

and

\section{Vlasta Bonačić-Koutecký}

Department of Chemistry, Humboldt-Universität zu Berlin, Brook-Taylor-Strasse 2, 12489 Berlin, Germany, vbk@chemie.hu-berlin.de

and Center of excellence for Science and Technology-Integration of Mediterranean region (STIM) at Interdisciplinary Center for Advanced Sciences and Technology (ICAST), University of Split, Meštrovićevo šetalište 45, HR-21000 Split, Republic of Croatia

100 p. 30 illus. in color.

\section{Marketing text:}

Metallic quantum clusters belonging to intermediate size regime between two and few hundred of atoms, represent unique building blocks of new materials. Nonlinear optical (NLO) characteristics of liganded silver and gold quantum clusters reveal remarkable features which can be tuned by size, structure and composition. The two-photon absorption cross sections of liganded noble metal quantum clusters are several orders of magnitude larger than that of commercially-available dyes. Therefore, the fundamental photophysical understanding of those two-photon processes in liganded clusters with few metal atoms deserve special attention, in particularly in context of finding the mechanisms responsible for these properties. A broad range of state-of-the-art experimental methods to determine nonlinear optical properties (i.e. two-photon absorption, two-photon excited fluorescence and second harmonic generation) of quantum clusters are presented. The experimental setup and underlying physical concepts are described.

Furthermore, the theoretical models and corresponding approaches are used allowing to explain the experimental observations and simultaneously offering the possibility to deduce the key factors necessary to design new classes of nanoclusters with large NLO properties. Additionally, selected studied cases of liganded silver and gold quantum clusters with focus on their NLO properties will be presented as promising candidates for applications in imaging techniques such as fluorescence microscopy or Second-Harmonic Generation microscopy. 


\section{About the Authors:}

Dr. Rodolphe Antoine is a Research director at the National Center for Scientific Research (CNRS). His research field is the study of molecular systems of growing complexity in the gas phase. In 1997, he was recruited at CNRS in Lyon for building a molecular beam deflection experiment for electric polarizability measurements on clusters and biomolecules. Most important results on biomolecules are: the first -and unique- measurements of electric dipole of isolated peptides and the first observation of gas phase beta sheets. In 2004, in order to go through larger and more complex systems, his research moved towards laser spectroscopy on trapped biomolecular ions and nanoparticles. He recently focused on the functional role of small silver and gold quantum clusters in model hybrid systems involving peptides in context of new generation of hybrid nanostructured materials for biosensing.

Prof. Dr. Dr. h.c. Vlasta Bonačić-Koutecký is professor of physical and theoretical chemistry in Department of Chemistry, Humboldt-Universität zu Berlin. Since 2010, she is the Director of the Interdisciplinary Center for Advanced Science and Technology (ICAST) and since 2014, she is a head of Center of excellence for science and technology - integration of Mediterranean region (STIM) at the University of Split in Croatia. Her major research areas are:

- Theoretical photochemistry and photophysics

- Chemistry and physics of metal clusters and cluster-biomolecule hybrids

- Development of cluster-based catalytic, optical and biosensing materials

- Quantum chemical adiabatic and nonadiabatic molecular dynamics "on the fly" in the frame of TDDFT

- Semiclassical dynamics

- Theory and methods for simulation of time-resolved spectroscopies

- Optimal control and laser selective photochemistry 


\section{Contents.}

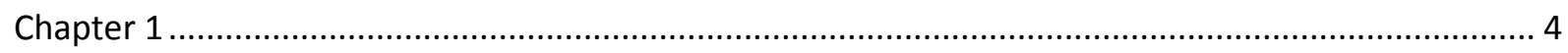

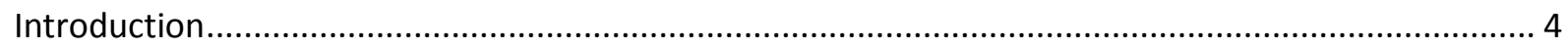

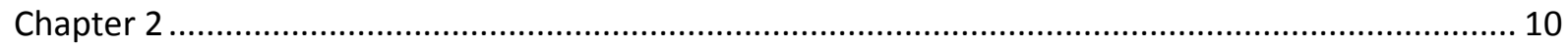

Liganded silver and gold quantum clusters: Background of their structural, electronic and optical

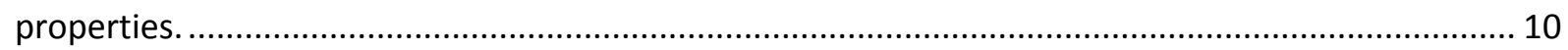

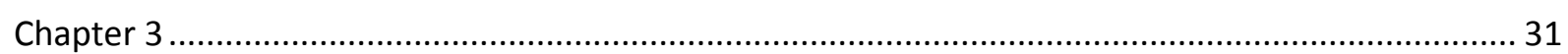

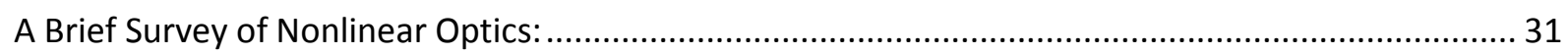

Second-Harmonic Generation and Two-Photon Absorption .......................................................... 31

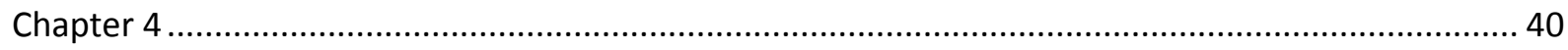

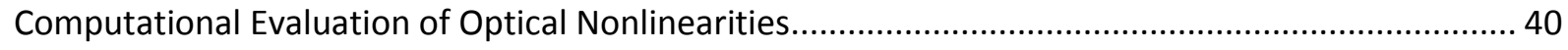

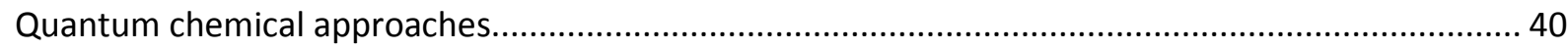

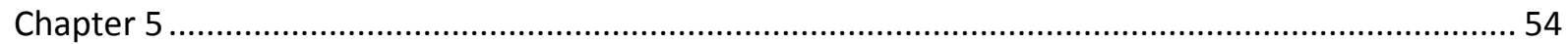

Design Strategy and Structure-Property Relation for Enhanced Two-Photon Absorption in Ligated

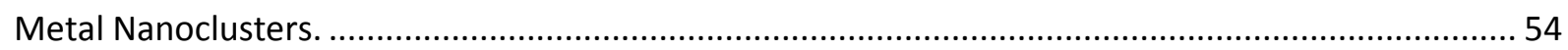

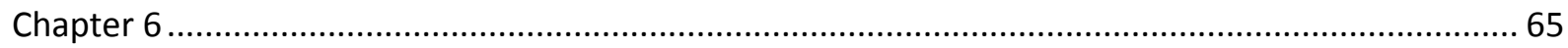

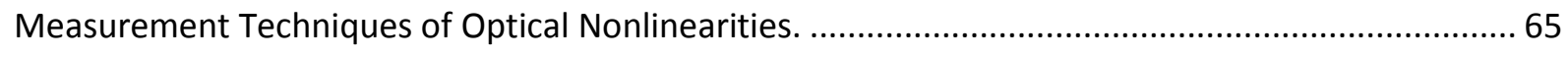

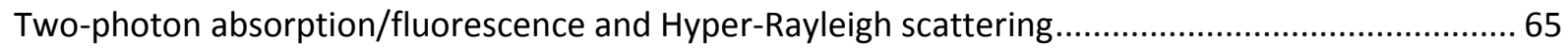

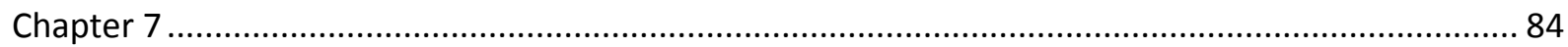

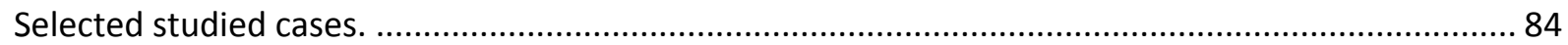

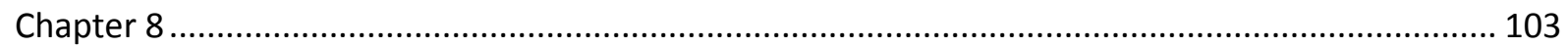

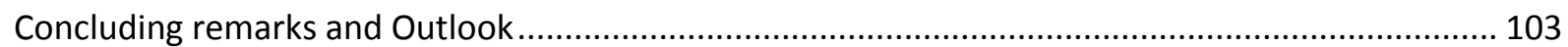




\section{Chapter 1}

\section{Introduction}

Metal clusters consist from two to several hundreds of metal atoms. Their properties differ substantially from both the constituting atoms and the bulk. Sub-nanometer gold and silver clusters exhibit size- and composition-dependent properties that are unique and differ from those of larger nanoparticles. The highly tunable properties of clusters by size and structure as well as and their high surface-to-volume ratio make them promising candidates for numerous technological applications.

Noble metal clusters, in particular silver and gold, display remarkable optical properties ${ }^{[1]}$ and have been widely applied in biolabelling, nanophotonics, light energy harvesting, etc. ${ }^{[2]}$ Furthermore, their properties exhibit non-scalable size dependence, which means that they vary with addition or removal of a single atom in a specific way that cannot be predicted. 
However, because "each-atom-counts" plays important role for determination of cluster properties, it is of crucial importance to develop robust synthesis methods to efficiently prepare metal clusters with a high "purity". ${ }^{[3]}$ By high purity, we mean that the metal clusters should be free of impurities and also with a unique chemical formula. For liganded metal quantum clusters presented in this book, it means that synthesis should lead ultimately to a cluster compound of a unique chemical formula $\mathrm{M}_{\mathrm{n}} \mathrm{L}_{\mathrm{m}}$ (where $\mathrm{M}$ is the metal and $\mathrm{L}$ is the ligand). Well-established characterization tools can use mass spectrometry to unambiguously determine their molecular weight, single-crystal X-ray crystallography to resolve their total structure with atomic precision, and NMR spectroscopy to probe both ligand environment and metal core chirality,....Complementary to experiment, theoretical approach is mandatory in order to provide additional information, in particular to the understanding of structureproperty relationships, which cannot be achieved experimentally and also to provide concepts that serve as basis for designing systems with desirable optical properties. And it is of paramount importance for an efficient joint experimental and theoretical investigation, to produce and characterize liganded metal quantum clusters with a high purity.

One of the key challenges in modern optics is a play with photons and colors, to achieve the highest specificity at the molecular level. Nonlinear optics due to drastic symmetry rules is able to provide high specificity in the light/matter interaction. ${ }^{[4]}$ The field of nonlinear optics has grown enormously in recent years due to the rapid technological advances that have occurred with smart ultrafast lasers. At low light intensities, typical of non-laser sources, the properties of materials remain independent of the intensity of illumination. Nonlinear optical (NLO) materials have a nonlinear response to the electric field associated with the light of a laser beam, leading to a variety of optical phenomena such as the generation of new light frequencies or the alteration of the material's optical properties. Light waves can then interact with each other, exchanging momentum and energy. This interaction of light waves can result 
in the generation of optical fields at new frequencies, including optical harmonics of incident radiation or sum- or difference-frequency signals. The simultaneous absorption of two photons by the same molecule was first analyzed theoretically in the 1930s by Maria Goeppert-Mayer $^{[5]}$ and was first demonstrated experimentally in $1961,{ }^{[6]}$ soon after the invention of the laser. Two-photon absorption (2PA) involves the simultaneous interaction of two photons, and so it increases with the square of the light intensity, whereas one-photon absorption depends linearly on the intensity. Using assumptions equivalent to those of the Beer-Lambert law, the attenuation of a beam of light resulting solely from 2PA is related to the molecular 2PA cross-section, usually reported in Goeppert-Mayer units $\left(1 \mathrm{GM}=10^{-50}\right.$ $\mathrm{cm}^{4}$.s.photons ${ }^{-1} \cdot$ molecule $\left.^{-1}\right)$.

Near infrared multiphoton microscopy is becoming a novel optical tool of choice for fluorescence imaging with high spatial and temporal resolution, diagnostics, photochemistry and nanoprocessing within living cells and tissues. ${ }^{[7,8]}$ As a result, there is a push for new kinds of contrast agents able to present high cross section for multiphoton absorption and high nonlinear emissive response... Considerable efforts have been made to engineer molecules with large nonlinear absorption cross sections. A class of molecules known as push-pull chromophores : these molecules consist of donor and acceptor end groups connected by a charge conjugation path, providing the possibility of a large charge transfer along the molecular axis upon excitation. ${ }^{[9]}$ However, such organic dyes may present poor water solubility and high cytotoxicity, Thus alternatives to organic fluorophores are highly desired.

Liganded metal quantum clusters present an original route for providing efficient chromophores for linear and nonlinear optics. Preparation of ultra-bright luminescent gold and silver clusters since 20 years provided a palette with rich diversity. ${ }^{[10,11]}$ The concept of a shell that protects the bare metal cluster and preserves its optical properties was first realized using gas matrices. ${ }^{[12,13]}$ Thanks to the diversity of (bio)organic chemistry, different templates 
have been proposed ranging from small organic molecules, polymers to large proteins, DNAs and even dendrimers. ${ }^{[14,15]}$ Thiolate molecules and proteins benefit from the specific binding between sulfur and gold or silver to induce formation of liganded metal quantum clusters (see Figure 1.1). Nonlinear optical (NLO) characteristics of such gold and silver clusters revealed remarkable features. ${ }^{[16]}$ The two-photon absorption cross section of protected noble metal nanoclusters is several orders of magnitude larger than that of commercially-available dyes (see figure 1.1). However, the fundamental photophysical mechanisms underlying those twophoton processes in ligand protected clusters with few metal atoms are not fully understood yet. Therefore, a joint theoretical and experimental effort is required in order to establish concepts for designing systems involving noble mental clusters with high nonlinear properties. The ligand molecules play a major role in enhancing the emissive properties of nanoclusters. ${ }^{[17]}$ Such nanoclusters can be viewed as a multi-shell system composed by a metal core, a metal-ligand interface, in particular with staple motifs leading to metal-sulfur bonds and the surface ligand molecules with their functional groups. These three shells may communicate in particular through charge transfer from ligand to metal core. We named this new class of NLO materials as "Ligand-Core" NLO-phores. ${ }^{[18]}$
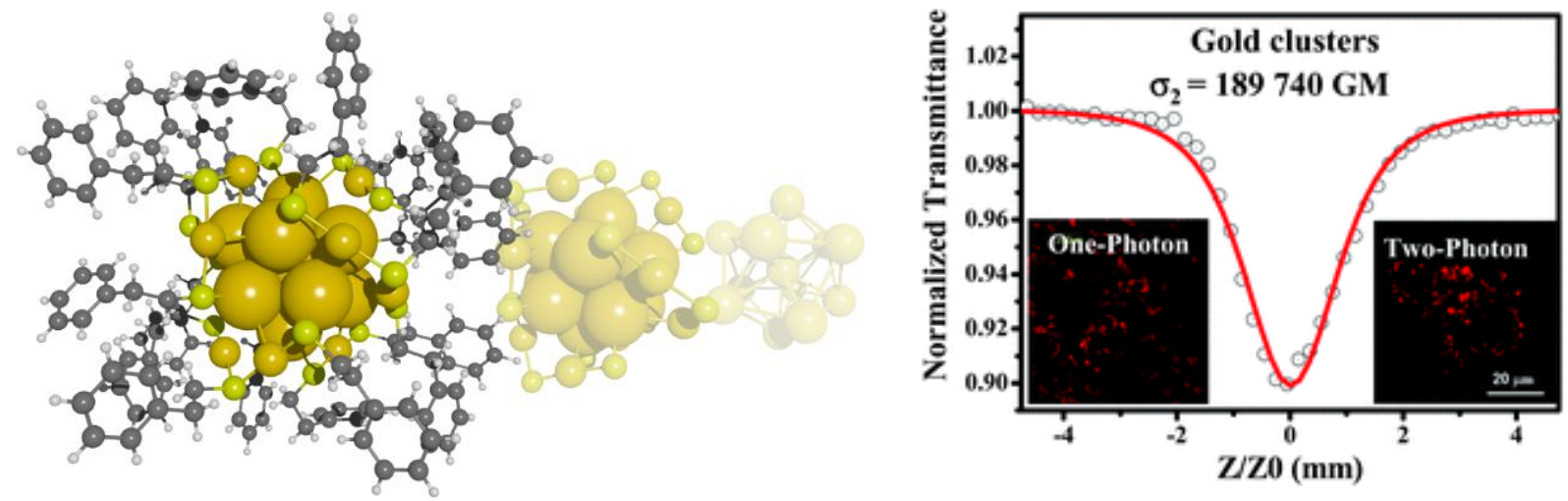

Figure 1.1 : (left) artistic view of ligated metal nanoclusters viewed as a "multi-shell system" with a metal core, a metalligand interface, and the surface ligand molecules. (right) z-Scan measurement results on the gold clusters, one-photon and two-photon excitation fluorescence images of SH-SY5Y neuroblastoma cells that were incubated with the gold clusters (adapted from ref. ${ }^{[19]}$ ). 
This book is organized in such a way that experimental and theoretical aspects are described in detail, so that the case studies discussed at the end of the book can easily be understood. Therefore, a background of liganded metal quantum clusters is first introduced. This is followed by brief survey of nonlinear optics (NLO) and in particular NLO techniques used for (bio)imaging application, e.g. second-harmonic generation and two-photon excited fluorescence. Then, quantum chemical approaches for evaluating hyperpolarizabilities and two-photon absorption cross sections of liganded metal quantum clusters will be described. This is followed by an in-depth exploration of strategy and structure-property relation for enhancing optical nonlinearities in ligated metal clusters. Then, the experimental methods to determine nonlinear coefficients will be described in detail. After discussing the theoretical and experimental methodology of optical nonlinearities in protected noble metal nanoclusters, we will demonstrate through selection of studied cases, how gold and silver nanoclusters can be utilized understand and predict their nonlinear optical properties as a function of their size and composition. Finally, a short summary and some perspectives liganded silver and gold quantum clusters as a new class of NLO materials will be presented.

\section{References.}

[1] U. Kreibig, M. Vollmer, Optical Properties of Metal Clusters, Springer Verlag, Berlin Heidelberg, 1995.

[2] in Frontiers of Nanoscience, Vol. Volume 9 (Eds.: T. Tsukuda, H. Häkkinen), Elsevier, 2015, p. ii.

[3] R. Jin, C. Zeng, M. Zhou, Y. Chen. Atomically Precise Colloidal Metal Nanoclusters and Nanoparticles: Fundamentals and Opportunities. Chemical Reviews 2016, 116, 10346.

[4] R. W. Boyd, Nonlinear Optics, Second Edition, 2003; and Third Edition 2008 ed., Academic Press, Boston, 1992.

[5] M. Göppert-Mayer. Über Elementarakte mit zwei Quantensprüngen. Annalen der Physik 1931, 401, 273.

[6] W. Kaiser, C. G. B. Garrett. Two-Photon Excitation in Ca\$ $\{\backslash$ mathrm $\{F\}\}\{2\} \$$ : $\$\{\backslash \text { mathrm }\{\mathrm{Eu}\}\}_{\wedge}\{2+\} \$$. Physical Review Letters 1961, 7, 229.

[7] K. König. Multiphoton microscopy in life sciences. Journal of Microscopy 2000, 200, 83. 
[8] H. Mojzisova, J. Vermot. When multiphoton microscopy sees near infrared. Current Opinion in Genetics \& Development 2011, 21, 549.

[9] F. Terenziani, C. Katan, E. Badaeva, S. Tretiak, M. Blanchard-Desce. Enhanced Two-Photon Absorption of Organic Chromophores: Theoretical and Experimental Assessments. Advanced Materials 2008, 20, 4641.

[10] M. A. H. Muhammed, T. Pradeep, in Advanced Fluorescence Reporters in Chemistry and Biology II: Molecular Constructions, Polymers and Nanoparticles (Ed.: A. P. Demchenko), Springer Berlin Heidelberg, Berlin, Heidelberg, 2010, pp. 333.

[11] I. Díez, R. H. A. Ras, in Advanced Fluorescence Reporters in Chemistry and Biology II: Molecular Constructions, Polymers and Nanoparticles (Ed.: A. P. Demchenko), Springer Berlin Heidelberg, Berlin, Heidelberg, 2010, pp. 307.

[12] S. Fedrigo, W. Harbich, J. Buttet. Collective dipole oscillations in small silver clusters embedded in rare-gas matrices. Physical Review B 1993, 47, 10706.

[13] G. A. Ozin, H. Huber. Cryophotoclustering techniques for synthesizing very small, naked silver clusters Agn of known size (where $n=2-5$ ). The molecular metal cluster-bulk metal particle interface. Inorganic Chemistry 1978, 17, 155.

[14] I. Diez, R. H. A. Ras. Fluorescent silver nanoclusters. Nanoscale 2011, 3, 1963.

[15] R. Jin. Atomically precise metal nanoclusters: stable sizes and optical properties. Nanoscale 2015, 7, 1549.

[16] S. H. Yau, O. Varnavski, T. Goodson. An Ultrafast Look at Au Nanoclusters. Accounts of Chemical Research 2013, 46, 1506.

[17] Z. Wu, R. Jin. On the Ligand's Role in the Fluorescence of Gold Nanoclusters. Nano Letters 2010, 10, 2568.

[18] I. Russier-Antoine, F. Bertorelle, N. Calin, Z. Sanader, M. Krstic, C. Comby-Zerbino, P. Dugourd, P.-F. Brevet, V. Bonacic-Koutecky, R. Antoine. Ligand-core NLO-phores: a combined experimental and theoretical approach to the two-photon absorption and two-photon excited emission properties of small-ligated silver nanoclusters. Nanoscale 2017, 9, 1221.

[19] L. Polavarapu, M. Manna, Q.-H. Xu. Biocompatible glutathione capped gold clusters as oneand two-photon excitation fluorescence contrast agents for live cells imaging. Nanoscale 2011, 3, 429. 


\section{Chapter 2}

\section{Liganded silver and gold quantum clusters: Background of their structural, electronic and optical properties.}

\section{Introduction.}

The purpose of this chapter is first to introduce physical background allowing one to gain understanding about connection between electronic energy quantization in metal nanoclusters and their optical properties. The optical response, in terms of electronic transitions whose positions and intensities are predicted by sophisticated quantum mechanical calculations, will be described. The concept of ligand protected metal clusters will be introduced and we then will summarize briefly the synthetic work on liganded quantum clusters of gold and silver and their characterization. Finally, we will discuss the link between their structural, electronic and optical properties. 


\section{Basics: from free electron model to electronic energy quantization in metal nanoclusters}

Along the atomic to bulk scale of noble metal systems, different properties and material behavior can be found. According to the number of metal atoms, classification into three size domains, corresponding to three different characteristic length scales, can be made: large nanoparticles, small nanoparticles and clusters (or nanoclusters). The wavelength of light is the first characteristic length scale encountered for these three size domains. For large metal nanoparticles (where their size is in the range of the wavelength of light), the optical properties can be quantitatively described with Mie theory for small metal spheres. ${ }^{[1]}$ Applying Maxwell's equations with appropriate boundary conditions in spherical coordinates, optical absorption, light scattering and extinction of a metal sphere depend on its volume and bulk dielectric functions. When particle size approaches the second characteristic length (where their size is much smaller than the wavelength of light), electron mean free path (the average distance an electron travels between two adjacent collisions, and $~ 50 \mathrm{~nm}$ for gold and silver), the dielectric function and refractive indices become size dependent. As a result, the optical responses such as plasmon absorptions of small metal nanoparticles have different size-dependences compared with large nanoparticles. Mie theory still can be extended into this quasi-static regime. In the third characteristic length, optical, electronic and chemical properties of metal clusters are dramatically different from the other two size regimes. In this smallest size regime, metal clusters become "molecular-like species", and discrete states with strong florescence can be observed. Recent progress in theory, in particular time-dependent density functional theory (TDDFT), allows for investigation of optical properties to be extended to more than 100 atoms for the simplest noble metal 
systems. This is size regime of small metal clusters with molecular-like properties that is the primary topic of this book.

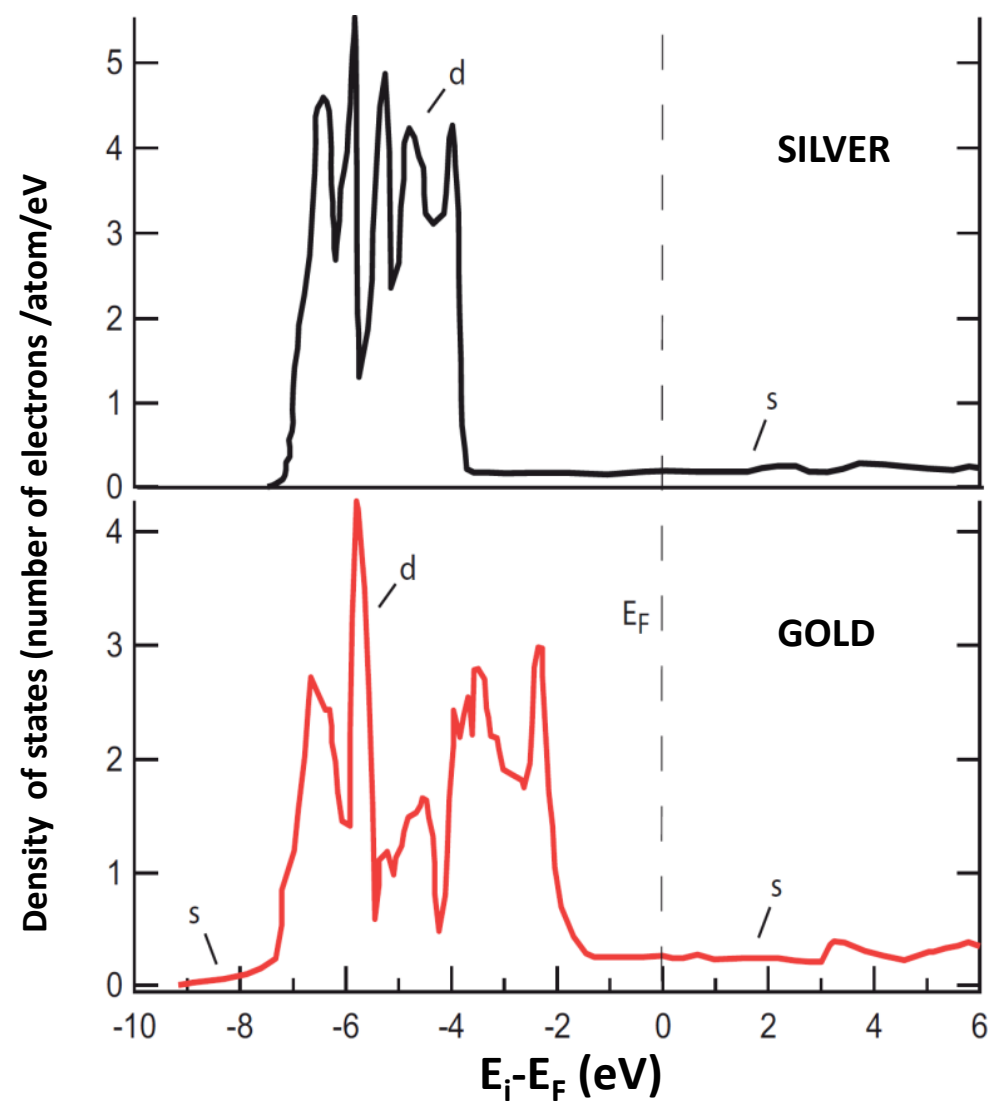

Figure 2.1 : Density of states (DOS) calculated for the noble metals Ag and Au. Adapted from refs. [2, 3].

Although optical responses of metals and metal nanoparticles are strongly size-dependent, metal band structure is only weakly size-dependent. Bulk metals have continuous band structures and the conduction bands are occupied with free electrons.

In Figure 2.1, the densities of states calculated for the silver and gold noble metals are compared. ${ }^{[2,3]}$ The d-type contributions dominate in contrast to the densities of states related to s-type electrons with lower intensities and smooth behavior which are located at left and right side of the d-states. However, in the case of gold, the electronic states of d-character are around $2 \mathrm{eV}$ below the Fermi level $\left(\mathrm{E}_{\mathrm{F}}\right)$, while in the case of silver they are located approximately $4 \mathrm{eV}$ below $\mathrm{E}_{\mathrm{F}}$. In addition, it is noteworthy that the band in gold is substantially broader than for silver and appears divided into two regions. Indeed, the 
relativistic effects such as spin-orbit coupling are particularly important in the case of gold and separation of angular momentum $\mathrm{j}=3 / 2$ and $\mathrm{j}=5 / 2$ is visible in the density of states. ${ }^{[3]}$ Such band structure is also observed from metal nanoparticles larger than $2 \mathrm{~nm}$, in which the density of states is large enough and energy level spacing between adjacent quantum states are much smaller than thermal energy. Thus, these delocalized free electrons coherently oscillate and give the well-known surface plasmon absorption. Differences in size-dependent optical response mainly arise from the change in the number of free electrons.

In order to understand the fundamental properties of metal nanoparticles, the free-electron theory represents a model which provides intuitive insight into the electronic properties of metal nanoparticles, in spite severe approximations such as neglection of electron-electron or electron-ion lattice interactions (both aspects are indeed fundamentally important). Nevertheless, the free-electron model is suitable for the first order approximation, and therefore we use this simple model to acquire some quantitative estimation of electronic energy quantization in noble metal nanoclusters.

The free electron model is neglecting the interactions between electrons. This approach of the electron gas without interaction allows the dispersion relation between energy / wave vector of electrons to be introduced and the concept of Fermi sphere. To determine the spectrum of permitted values of the electronic wave vector, one has to consider a three-dimensional cubical box that has a side length $a$. The surfaces of constant energy are spheres with radius $\mathrm{k}=\sqrt{2 \mathrm{mE}} / \hbar$. System with $\mathrm{N}$ electrons is occupied according to the Pauli principle at $T=0$ all states within the 'Fermi sphere' with radius $k_{F}$ and volume $\frac{4}{3 \pi \mathrm{k}_{\mathrm{F}}^{3}}$ are occupied. This defines the Fermi surface with the Fermi energy $E_{F}=\frac{\hbar^{2} k_{F}^{2}}{2 m}$ and the Fermi wave vector $k_{F}$. The Fermi energy is approximately $5.5 \mathrm{eV}$ for gold and silver.

For small systems, the number of atoms is finite, thus the spacing $\delta$ becomes noticeable and increases with decreasing cluster size. If we use the thermal energy $\left(k_{B} T\right)$ at room temperature 
as a criterion, using $\delta=\frac{4 \pi^{2}}{\mathrm{a}^{3}}\left(\frac{\hbar^{2}}{2 \mathrm{~m}}\right)^{\frac{3}{2}} \mathrm{E}^{-\frac{1}{2}}$ we can estimate at what size electronic energy quantization will be comparable with thermal energy, that is, $\delta=\mathrm{k}_{\mathrm{B}} \mathrm{T}$. By substituting all the constants (SI units) and the highest occupied energy level of gold (i.e. the Fermi level, $\mathrm{E}_{\mathrm{F}} \sim 5.5$ $\mathrm{eV}$ ), the corresponding particle volume $\left(a^{3}\right)$ is $\sim 5 \times 10^{-27} \mathrm{~m}^{3}$ (thus an equivalent particle size $\sim 1.7 \mathrm{~nm}$ ). The number of valence electrons in the system is estimated to be about $280 .{ }^{[4]}$ Thus, one would expect electronic energy quantization to occur when the number of $6 \mathrm{~s}$ valence electrons in a cluster drops to about 200-300.

\section{Metal optical responses at different size scales}

The optical response of free-electron metals in the bulk state is well described by DrudeLorentz-Sommerfeld/free electron model. ${ }^{[5]}$ Based on this model, the macroscopic optical response of metals follows by summing the single electron response over the total number of the free electrons. Thus, according to this model, all the free electrons in a metal react in phase with the electrical field. In reality, due to the large electron density and delocalization, all the free electrons indeed coherently oscillate under a time dependent electrical field, which gives the well-known bulk plasmon absorption.

In general, when the particle size decreases, the dielectric function begins to strongly depend on R. For small clusters of less than $10 \mathrm{~nm}$ size, model Mie-Drude is no longer appropriate to discuss absorption spectra in the details. The geometry of the clusters must be determined by quantum chemical methods that often use group theory and the optical response is described in terms of electronic transitions whose positions and intensities are predicted by sophisticated quantum mechanical calculations. As an illustration, the absorption spectra obtained with the $a b$ initio EOM-CCSD and STEOM-CCSD methods accounting for electron calculation for the most stable structures of $\mathrm{Ag}_{5-8}$ are given in Fig. 2.2. ${ }^{[6]}$ This pioneering work is a nice illustration of the molecular-like behavior of nanoclusters leading to an 
electronic energy quantization and the changes in the leading features of the patterns as a function of the cluster size and corresponding structure.

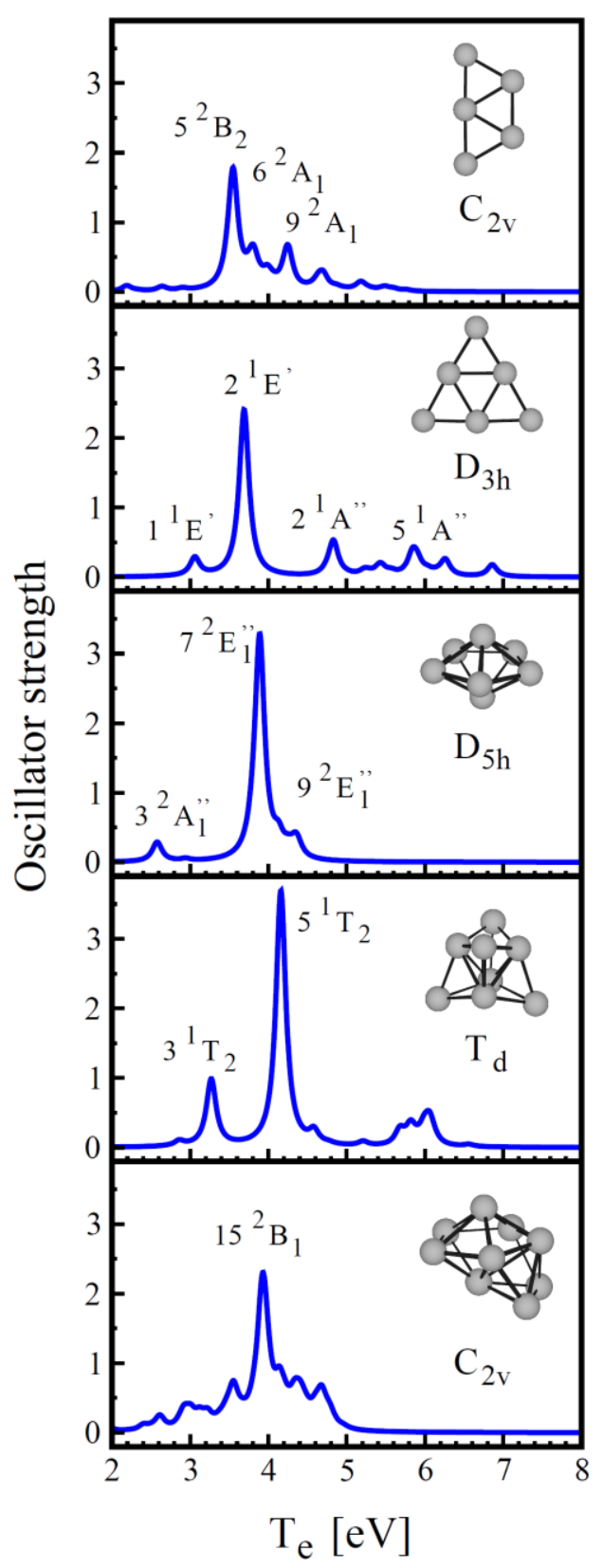

Figure 2.2 : Optically allowed transitions Te in eV and oscillator strengths $f$ e obtained from EOM-CCSD and STEOM-CCSD calculations using 11e-RECP with associated $\mathrm{AO}$ basis for the lowest energy structures of $\mathrm{Ag}_{5-8}$. most stable structure of $\mathrm{Ag}_{9}$ cluster obtained from the RPA method. The lines have been broadened using Lorentzian function with the width of $0.15 \mathrm{eV}$. Adapted from ref. [5]. 


\section{Time dependent density functional theory (TDDFT)}

Recent progress in computation methods, in particular within TDDFT approach, allows the investigation of optical properties of simplest noble metal systems to be extended to more than 100 atoms. TDDFT can be viewed as an exact reformulation of time-dependent quantum mechanics, where the fundamental variable is no longer the many-body wave function but the density. This time-dependent density is determined by solving an auxiliary set of the KohnSham equations. The important part of the many-body interaction is included in the so-called exchange-correlation potential, for which adequate approximations have been developed. If the external time-dependent potential is "small," complete numerical solution of the timedependent Kohn-Sham equations can be replaced by the linear response theory allowing to calculate photoabsorption spectra of relatively large systems.

In spite of the well-known approximations for electronic correlations within TDDFT and inclusion of only single excitations as well as the influence of the choice of the functionals on the accuracy, theoretical results for absorption are in acceptable agreement with the available experimental findings, as illustrated on Fig. 2.3. ${ }^{[7]}$ 

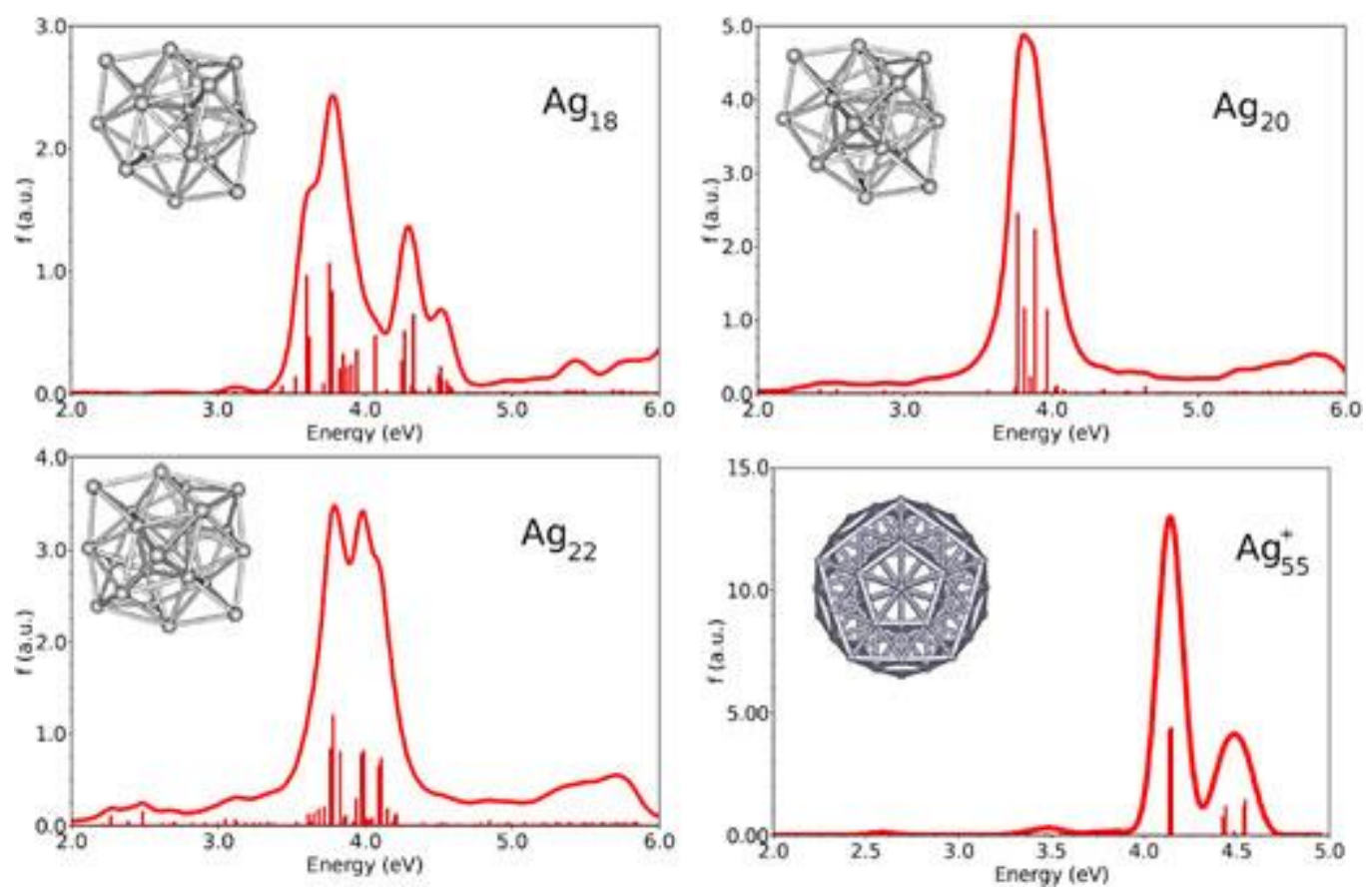

Figure 2.3 : Absorption spectra of pure silver clusters (Agn, $n=18-55$ ) investigated in the framework of the timedependent density functional theory (TDDFT) with the use of the functional CAM-B3LYP. Adapted from ref. [7].

\section{Optical response from noble metal clusters}

\section{a) Absorption and emission of bare gold clusters}

In 1987, Markus and Schwentner first observed that gold dimers in rare gas matrices yield 450, 545 and $580 \mathrm{~nm}$ emission. ${ }^{[8]}$ Harbich et al. also used a similar method to prepare $\mathrm{Au}_{2}$, and $\mathrm{Au}_{3}$ embedded in argon matrices and studied their optical response at low temperature. ${ }^{[9,}$ ${ }^{10]}$. Absorptions centered 235, 263, 285, 310 and $345 \mathrm{~nm}$ are clearly distinguishable and two emission regions centered and $\sim 453 \mathrm{~nm}$ and $510 \mathrm{~nm}$ were observed from $\mathrm{Au}_{3}{ }^{[9]}$ Collings et al. also investigated the gas phase absorption spectra of gold clusters $\mathrm{Au}_{\mathrm{n}}$ ( $\mathrm{n}=7,9,11,13)$ and their cations using photodepletion spectroscopy. ${ }^{[11]}$ Sharp lines between 1.9 and $5.6 \mathrm{eV}$ in absorption bands were observed.

\section{b) Absorption and emission of bare silver clusters}

Kreibig studied the evolution of optical absorption of silver along the size scale from atoms over clusters to bulk. Discrete electronic transitions instead of a collective plasmon were 
observed for very small $\mathrm{Ag}_{\mathrm{n}}$ clusters at low temperature, while the common metal properties were present in the case of silver systems embedded in glass which contain more than about 400 atoms. ${ }^{[12]}$ In 1978, Ozin and Huber used cryo-photo-clustering technique to prepare small silver clusters $\operatorname{Ag}_{\mathrm{n}}$ (where $\mathrm{n}=2-5$ ) within Ar matrices at 10-20 K. Discrete absorption bands between 3.1 and $4.1 \mathrm{eV}$ were observed from few atom silver clusters at low temperature. Later, using "soft landing" mass spectrometry, ${ }^{[13]}$ Harbich and coworkers were able to prepare size selected silver dimers and trimers in solid Ar matrices, and investigated their absorptions and fluorescence. $\mathrm{Ag}_{3}$ clearly shows absorptions at 318, 386 and $495 \mathrm{~nm}$ and had discrete emissions at 374, 616 and $705 \mathrm{~nm}$. Notice that in the case of size-selected clusters such as $\mathrm{Ag}_{8}$ and $\mathrm{Ag}_{9}$ different isomeric structures close in energy are presented and their contribution has to be taken into account for interpretation of measured absorption spectra of these species. ${ }^{[14]}$ Complementary to the studies on isolated silver clusters, Chen and coworkers $^{[15]}$ used zeolites to prepare fluorescent silver clusters at room temperature and Dickson and coworkers ${ }^{[16]}$ produced, by photoreducing silver oxide films, fluorescent silver clusters. Solid $\mathrm{Kr}$ matrices, zeolites, or silver oxides increase cross section of irradiative decay and prevent dissociation of silver clusters, even though the excitation energies are comparable to the photodissociation energies of naked silver clusters. Indeed, $\mathrm{Ag}$ and $\mathrm{Ag}_{2}$ loss are a general phenomenon for photodissociation of $\operatorname{Ag}_{n}$ clusters. Hild and coworkers ${ }^{[17]}$ found that after excitation by photons with energies $1.5 \sim 4 \mathrm{eV}$, silver clusters decay by emission of neutral atoms or dimers with lifetimes in the range $100 \mu$ s to $15 \mathrm{~ms}$.

\section{Role of ligand protection. Atomically precise clusters of gold and silver}

The use of solid gas or inorganic matrices permits to protect gold or silver clusters from photodissociation. Also, the large excited state charge separation is also a possible process in the photophysics of metal clusters composed by few atoms. Organic scaffolds allow the 
formation and stabilization of metal clusters in solution. The use of organic scaffolds for fluorescent metal nanoclusters are relatively new, first reported by the group of Dickson in 2002 for silver nanoclusters. ${ }^{[18]}$ These organic scaffolds have tremendous potentials, as the interaction between the ligands and metal clusters can be adjusted leading to tunability in their spectroscopic properties. For example by using DNA oligomers as organic scaffolds and by playing with the nucleotide sequence of DNA oligomers it is possible to synthesize silver nanoclusters that emit from the blue to near-infrared region. ${ }^{[19]}$

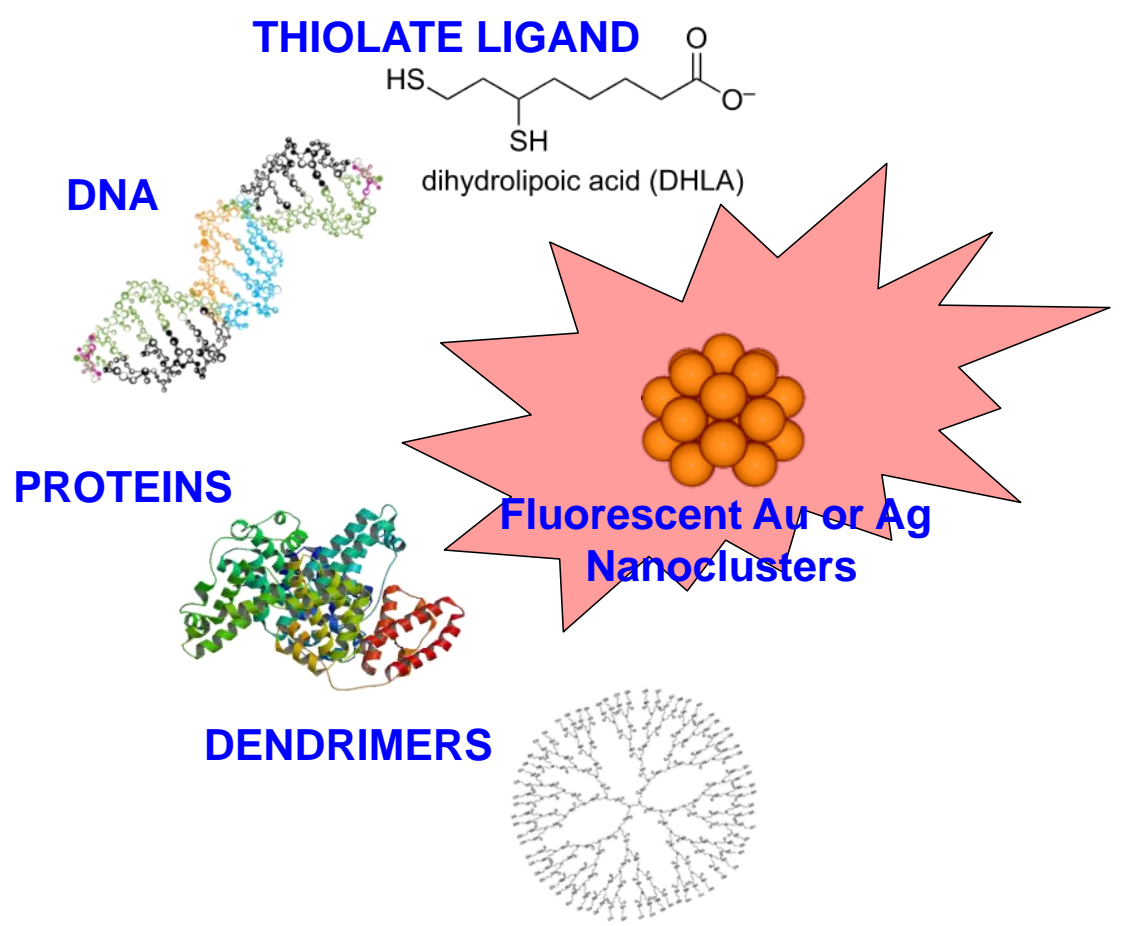

Figure 2.4 : Protected metal nanoclusters with possible scaffolds.

Ligands play very important roles in the formation of NCs as protective agents, which can prevent the metal clusters from aggregation and then keep the size-dependent fluorescence property. The formation and stabilization of gold or silver nanoclusters in solution have been accomplished in various ways (see Fig. 2.4). The proper choice of parameters for the reaction, including the temperature, the reducing method, the stabilizers and the initial ratio of metal salt:stabilizer, plays a crucial role for the successful synthesis of nanoclusters and to 
limit the size to few-atom nanoclusters. In addition to the ultrasmall size, the ligands used for NCs preparation also have impacts on their fluorescence properties. Jin and coworkers ${ }^{[20]}$ demonstrated that for gold NCs, the surface ligands of NCs not only can be used as capping agent but also largely affect the fluorescence of NCs through charge transfer from surface ligand to the gold core. If the surface ligands are strong electron donors, the fluorescence can be enhanced. In fact, the ligands with electron-rich atoms or groups have been found to be an effective choice of surface ligands to enhance the fluorescence of NCs.

\section{a) Synthetic routes}

The production of silver and gold nanoclusters can be performed following several routes. The metal ions from dissolved metal salts can be reduced, either by a chemical reductant (e.g.sodium borohydride, ...), (see fig. 2.5a) by light (photoreduction with near-ultraviolet light) or by $\gamma$-rays (by radiolysis of water). The chemical reduction and the photoreduction are the most commonly used methods. ${ }^{[21]}$ The specific properties of metal nanoclusters, such as the composition, stability and fluorescence quantum yield, depend largely on the scaffold used during reduction.

Thiols are frequently used on noble metal substrates because of the strong affinity of sulfur for these metals. Also, thiolated ligands (-SR) have appeared to be extremely good candidates to produce ultrasmall nanocluster sizes, in particular for gold. ${ }^{[4]}$ Following the pioneering work of Brust et al. ${ }^{[22]}$ based on the reduction of the metal precursors and the formation of metal core, thiol-containing small molecules were extensively used to stabilize gold nanoclusters in the aqueous solution. The use of thiol-containing small molecules as stabilizers permits to better control of production of AuNCs than phosphine-capped ones, contributing to the stronger Au-S covalent bonding. Generally, the method of synthesizing thiolate-capped AuNCs has processes as follows. Gold salts $\left[\mathrm{AuCl}_{4}\right]^{-}$are dissolved in water and then transferred to an organic solvent by phase transfer agent; the thiols are added to the 
mixture inducing reduction of $\mathrm{Au}^{3+}$ ions into $\mathrm{Au}^{+}$ions and form $\mathrm{Au}^{+}-\mathrm{SR}$ complexes or polymers; then the $\mathrm{Au}^{+}$polymers are reduced by adding the reducing agent and lead to thiolate-protective gold nanoclusters.

Glutathione (GSH), a ubiquitous low-molecular weight thiol, played a significant role in producing gold NCs which showed good water solubility, bioactive surface, and high stability. It has been widely investigated for protecting the $\mathrm{Au}^{3+}$ ions when they were being reduced by sodium borohydride $\left(\mathrm{NaBH}_{4}\right) \cdot{ }^{[23-25]}$ Whetten and coworkers have proposed an unprecedented thiol-protective AuNC route by using the GSH (N- $\gamma$-glutamyl-cysteinylglycine) as the stabilizer. ${ }^{[25]}$ The unpurified synthesized (as-prepared) AuNCs were fractionated by using polyacrylamide gel electrophoresis (PAGE) and characterized by mass spectrometry (MS) [102]. Tsukuda and colleagues have also reported the characterization of fractionated AuNCs protected by GSH monolayers. The as-prepared AuNCs were isolated into single-sized $\mathrm{Au}_{\mathrm{n}}(\mathrm{SR})_{\mathrm{m}}$ clusters by the PAGE method and characterized using electrospray mass spectrometry. ${ }^{[23,24]}$

While the routes for producing metal $\mathrm{NCs}$ lead to a mixture of $\mathrm{Au}_{\mathrm{n}}(\mathrm{SR})_{\mathrm{m}}$ cluster size, achieving atomic precision and molecular purity is challenging because the nanocluster growth is extremely complicated and remains poorly understood. Nevertheless, a systematic methodology called "size focusing" (see fig 2.5b), for achieving size specific gold and silver clusters with molecular purity has been proposed. This methodology consists of two primary steps, (i) kinetically controlled synthesis of an $\mathrm{M}_{\mathrm{n}}(\mathrm{SR})_{\mathrm{m}}$ mixture with a properly controlled size range and (ii) thermodynamically dictated size-focusing of the mixture to single-sized nanoclusters. In parallel, a new approach, which is to utilize ligand exchange to induce size and structure transformation and, hence, to attain new $\mathrm{M}_{\mathrm{n}}(\mathrm{SR})_{\mathrm{m}}$ nanoclusters was also proposed (see fig. 2.5c). ${ }^{[26]}$ 


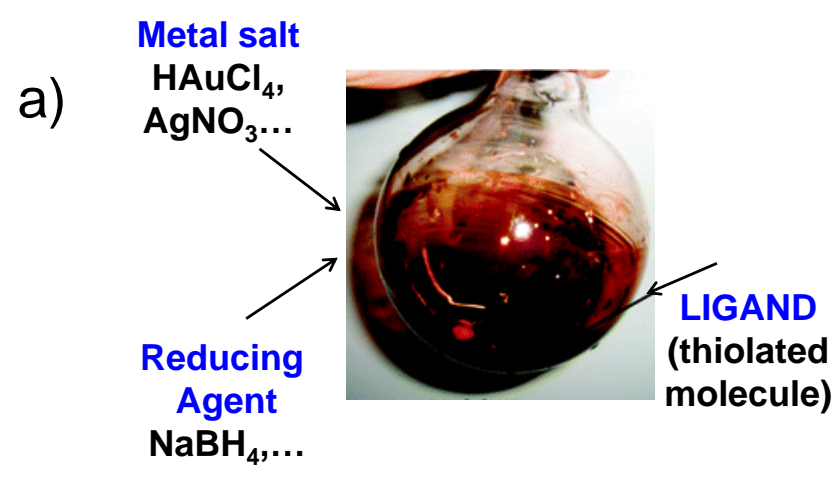

b)
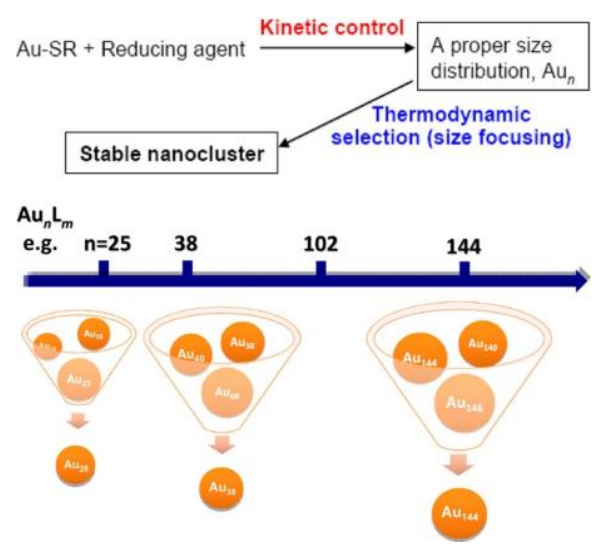

c)

$\mathrm{Au}_{\mathrm{n}}(\mathrm{SR})_{\mathrm{m}}$

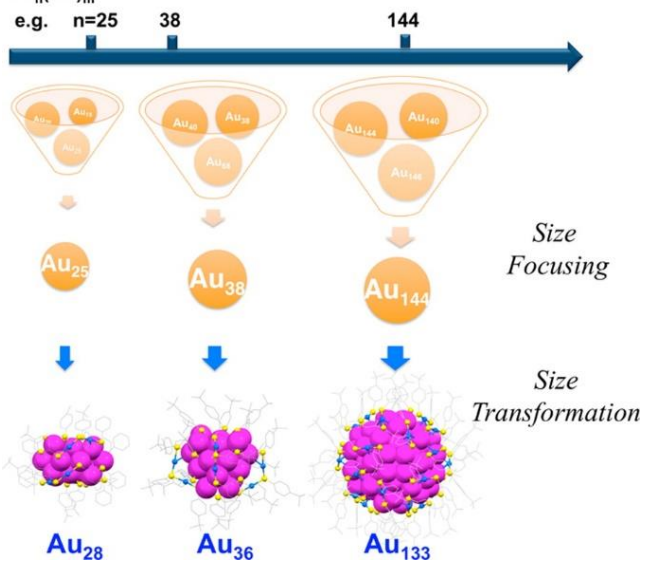

Figure 2.5: a) General route to produce thiolate protected nanoclusters by a chemical reductant. Schematic Diagrams of b) Size-Focusing Methodology and c) ligand exchange to induce size and structure transformation. Adapted from ref.[27]

\section{b) Characterization, structural, electronic and optical properties}

Concerning the characterization of the ultrasmall NCs, UV-Vis absorption spectroscopy, FTIR, CD and XPS are frequently utilized techniques. FTIR can be used to examine the structures of ligands and NCs, through the loss of S-H stretching vibration. These results inferred that the Metal-S bond was formed and that the NCs embedded in the template would 
not affect the surface structure of the template. The oxidation state of the metal atoms of the core can be examined by XPS. For gold, the peaks of $84.0 \mathrm{eV}$ and $84.9-85.3 \mathrm{eV}$ corresponded with the binding energy of $\mathrm{Au}(0)$ and $\mathrm{Au}(\mathrm{I})$, which indicated that $\mathrm{Au}(\mathrm{III})$ was reduced by the capping agents. (This sentence is not understandable) In UV-Vis absorption spectroscopy, the disappearance of the plasmon-absorption band is an indication of the formation of nanoclusters. The absorption bands of NCs are related to ligand and core size. The optical spectra of well-defined $\mathrm{Au}_{\mathrm{n}}(\mathrm{SR})_{\mathrm{m}}$ nanocluster are particularly useful in the routine syntheses, as the spectra provide a quick diagnosis of the crude product prior to mass spectrometric analysis. ${ }^{[28]}$

Mass spectrometry based methods, e.g. MALDI-TOF-MS and ESI-MS were carried out to analyze the molecular weight of the ultra small NCs. ${ }^{[29]}$ The use of traditional weak organic acid MALDI matrices has provided a means to measure the mass of the core of ligandprotected NCs; however, these matrices do not prevent extensive ligand fragmentation. The first MALDI spectra of intact of $\mathrm{Au}_{25}(\mathrm{SCH} 2 \mathrm{CH} 2 \mathrm{Ph})_{18}$ using various matrices, were published by Dass et $a l .{ }^{[30]}$ The use of glutathione as a ligand and its water-solubility provides an opportunity to purify the cluster by running it through polyacrylamide gel electrophoresis (PAGE). The PAGE purification enabled the isolation of intact clusters, as demonstrated by Tsukuda et al. ${ }^{[23]}$ for gold NCs and Bigioni et al. ${ }^{[31]}$ for silver NCs.

However, due to a large amount of chemical noise and/or unresolved isotopic pattern and limited mass accuracy of MS instruments, alternate molecular formula with a very similar mass can lead to misleading formula. This underscores two challenges of peak identification from intact NCs, namely that multiple molecular formulae can be nearly isobaric, and that impurities or ligand fragmentation can generate a large amount of chemical noise which frustrates the assignment of atomic information. Clean samples and instrumentation with very high resolving power decreases the uncertainty of molecular formula assignments, since the 
number of indistinguishable, nearly-isobaric permutations are decreased. Our experimental group has pushed forward the isotope-resolved mass spectrometry to solve the molecular formula of ultrasmall NCs. ${ }^{[32]}$ Examples of experimental and calculated isotopic distributions for gold-glutathione $\mathrm{Au}_{\mathrm{n}} \mathrm{SG}_{\mathrm{m}}$ clusters are displayed in Fig. 2.6. The observed charge $\mathrm{z}$ of a gold-glutathione complex in the mass spectrum is the sum of the net charge $q$ of the complex plus the charge due to deprotonation of glutathione side chains. The observed charge of the complex and the number of deprotonation are both determined from the isotope-resolved mass spectrum resulting in a direct determination of the net charge q. Note that mass spectrometric identification of precise formulas of silver nanoclusters are still nontrivial because of the limited stability of $\operatorname{Ag}_{n}(S R)_{m}$ nanoclusters and the rich isotopic pattern that significantly broaden mass peaks caused by silver isotopes.
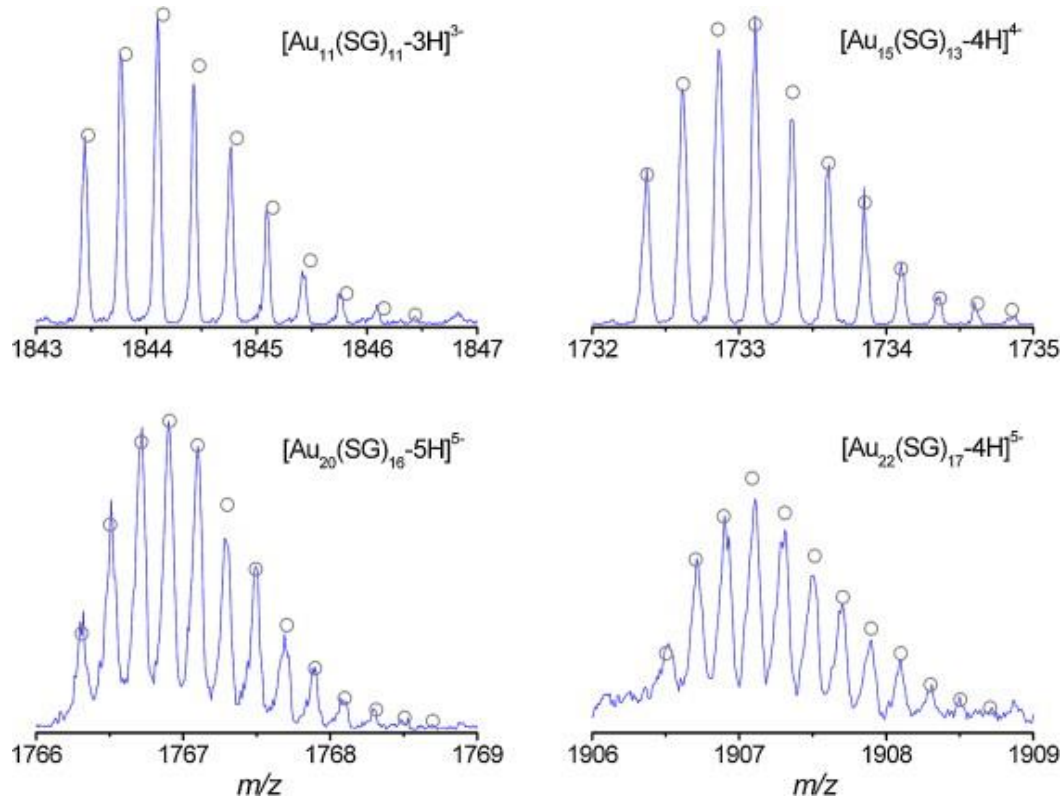

Figure 2.6: Experimental isotopic distribution recorded for $A_{11}(S G)_{11}$ (charge state 3-), $A_{15}(S G)_{13}$ (charge state 4-), $\mathrm{Au}_{20}(\mathrm{SG})_{16}$ (charge state 5-) and $\mathrm{Au}_{22}(\mathrm{SG})_{17}$ (charge state 5-). Circles show the theoretical isotopic distributions. Adapted from ref. ${ }^{[32]}$.

X-ray crystallography is the "holy-grail" technique to solve the crystal structure of nanoclusters and reveals the nature of bonding and the packing of atoms. The first breakthrough was achieved by Kornberg and coworkers when they reported the synthesis and 
total structure determination of $\mathrm{Au} \mathrm{NCs}$ comprising 102 gold atoms and 44 p-mercaptobenzoic acid. ${ }^{[33]}$ Since then, more structures have been achieved. A summary of the reported $\mathrm{Au}_{\mathrm{n}}(\mathrm{SR})_{\mathrm{m}}$ structures is given in the excellent recent review of Rongchao Jin. ${ }^{[27]}$ Here are selected $\mathrm{Au}_{\mathrm{n}}(\mathrm{SR})_{\mathrm{m}}$ nanoclusters that have been synthesized in high purity and adequately characterized : $\mathrm{Au}_{15}(\mathrm{SR})_{13}, \mathrm{Au}_{18}(\mathrm{SR})_{14}, \mathrm{Au}_{20}(\mathrm{SR})_{16}, \mathrm{Au}_{22}(\mathrm{SR})_{16,17,18}, \mathrm{Au}_{23}(\mathrm{SR})_{16}, \mathrm{Au}_{24}(\mathrm{SR})_{20}$, $\mathrm{Au}_{24}(\mathrm{SR})_{16}, \mathrm{Au}_{25}(\mathrm{SR})_{18}{ }^{\mathrm{q}}(\mathrm{q}=-1,0,+1), \mathrm{Au}_{28}(\mathrm{SR})_{20}, \mathrm{Au}_{30} \mathrm{~S}(\mathrm{SR})_{18}, \mathrm{Au}_{36}(\mathrm{SR})_{24}, \mathrm{Au}_{38}(\mathrm{SR})_{24}$, $\mathrm{Au}_{38} \mathrm{~S}_{2}(\mathrm{SR})_{20}, \mathrm{Au}_{40}(\mathrm{SR})_{24}, \quad \mathrm{Au}_{44}(\mathrm{SR})_{28}, \mathrm{Au}_{52}(\mathrm{SR})_{32}, \quad \mathrm{Au}_{55}(\mathrm{SR})_{31}, \mathrm{Au}_{64}(\mathrm{SR})_{32}, \mathrm{Au}_{67}(\mathrm{SR})_{35}$, $\mathrm{Au}_{99}(\mathrm{SR})_{42}, \quad \mathrm{Au}_{102}(\mathrm{SR})_{44}, \quad \mathrm{Au}_{104}(\mathrm{SR})_{41}, \quad \mathrm{Au}_{130}(\mathrm{SR})_{50}, \quad \mathrm{Au}_{133}(\mathrm{SR})_{52}, \quad \mathrm{Au}_{137}(\mathrm{SR})_{56}, \quad$ and $\mathrm{Au}_{144}(\mathrm{SR})_{60 .}{ }^{[27]}$ On the other hand, here are some selected $\mathrm{Ag}$ nanoclusters protected by thiolates: $\operatorname{Ag}_{11}(\mathrm{SR})_{7}, \mathrm{Ag}_{15}(\mathrm{SR})_{11},\left[\mathrm{Ag}_{17}(\mathrm{SR})_{12}\right]^{3-}, \quad\left[\mathrm{Ag}_{25}(\mathrm{SR})_{18}\right]^{-}, \operatorname{Ag}_{29}(\mathrm{SSR})_{12}, \mathrm{Ag}_{30}(\mathrm{SR})_{18}$, $\mathrm{Ag}_{31}(\mathrm{SR})_{19}, \quad\left[\mathrm{Ag}_{32}(\mathrm{SR})_{19}\right]^{0},\left[\mathrm{Ag}_{35}(\mathrm{SR})_{18}\right]$ and $\left[\mathrm{Ag}_{44}(\mathrm{SR})_{30}\right]_{4-\cdot}{ }^{[27]}$ Note that some bimetal nanoclusters (AuAg, $\mathrm{AuCu}, \mathrm{AuPt}, \ldots .$.$) and other gold nanoclusters protected by various$ types of ligands (phosphine; diphosphine; selenolate; alkynyl) withcrystal structures were solved by X-ray crystallography. ${ }^{[27]}$ However, the extensive application of X-ray crystallography as a characterization technique is limited, because it is often challenging to produce sufficiently high-quality crystals for a solvent. Indeed, the stability of water soluble glutathione monolayer protected gold and silver clusters has always been ambiguous at high temperature. The hydrolysis of glutathione (GSH) to pyroglutamic acid has always been observed in the ESI-mass spectrometry of these clusters. The fragile nature of these clusters has always been a concern not limited to mass spectrometric studies. The stability of water soluble glutathione monolayer protected gold and silver clusters, has also prevented it from any type of crystallization studies. Although the metal core system is stable, the nature of glutathione ligand plays an important role in its stability. Glutathione (GSH) is a tripeptide ( $\gamma$-Glu-Cys-Gly). It exists as disulphide GSSG. The bulky size of this ligand, easily hydrolysable amide bond, and stability restricted to lower temperatures makes it vulnerable 
to different degradation process. The difficulty to extract quantitative information from the diffraction patterns of nanosized and poorly crystallized compounds is the reason why X-ray Powder Diffraction (XRPD) techniques were mostly used to support the structural and microstructural features obtained by other techniques such as electron microscopy.

Also, NMR can be an important and indispensable tool for gaining insight into the structure of gold clusters. Jin and coworkers have demonstrated that the complicated 1D 1H NMR spectrum of $\mathrm{Au}_{25}(\mathrm{SG})_{18}$ clusters, with the aid of 2D NMR experiments (COSY and HSQC), can provide a wealth of information and facilitate identification of the structure of $\mathrm{Au}_{25}(\mathrm{SG})_{18}$ clusters when combined with mass spectrometry and optical spectroscopy. ${ }^{[34]}$

The X-ray structures of nanoclusters reveals that the surface thiolate ligands (-SR) do not simply passivate the gold core, instead, they form unique oligomeric units such as $\mathrm{Au}(\mathrm{SR})_{2}(-$ $\mathrm{RS}-\mathrm{Au}-\mathrm{RS}-)$ and $\mathrm{Au}_{2}(\mathrm{SR})_{3}(-\mathrm{RS}-\mathrm{Au}-\mathrm{S}(\mathrm{R})-\mathrm{Au}-\mathrm{SR}-)$ (called staples or semi-rings), which bind to the surface (i.e. core-surface) gold atoms for protection; in other words, the staple units are anchored to the core by sulfur in atop positions. Interestingly, The $\mathrm{Ag}_{44}$ structure was found to be quite different from that of the same size $\mathrm{Au}_{44}$ nanocluster; thus, it was thought that Ag nanoclusters might follow quite different structural rules than gold nanoclusters. Silver nanoclusters exhibit more complex surface structures than gold, in particular, Ag and SR can assemble into three-dimensional structures.

Finally concerning optical properties, strongly emissive nanoclusters are highly desirable. Strategies such as aggregation-induced fluorescence, silver doping and ligand-shell rigidifying for increasing the PL quantum yield and tuning the PL colors of nanoclusters have been proposed. However, the origins of PL in nanoclusters are still not fully understood. The ligand molecules along with the geometric and electronic structures of the metal core play a major role in enhancing emissive properties. Such nanoclusters can be viewed as a "multishell system" (see fig. 2.7) composed by (1) a metal core, (2) a metal-ligand interface, in 
particular with staple motifs leading to metal-sulfur bonds, and (3) the surface ligand molecules. These three shells may communicate in two different ways : charge transfer from ligand to metal core (analogy with ligand-to-metal charge transfer (LMCT) or ligand-to metal- metal charge transfer (LMMCT) observed in metal complexes) and through direct bonding or direct donation of delocalized electrons of electron-rich groups of the ligands. ${ }^{[20]}$ The last ten years, some general trends have been figured out concerning the de-excitation pathways following a visible or near-UV absorption. The following experimental and theoretical findings were assembled from the present work and literature to derive the energy diagram in Fig. 2.7.

Near-ultraviolet and visible absorbance may arise transitions between molecular orbitals with either high ligand contributions or high metal character (LMCT), as well as from metal-metal electronic transitions. A rapid ( $<1$ ps lifetime) decay pathway for clusters which have a core of metal atoms, that may lead to an emission in the visible. A long-lived $(>100$ ns lifetime), charge-transfer component is present for all clusters. NIR emission in the clusters is related to the surface states and originates from the charge transfer excited state. 


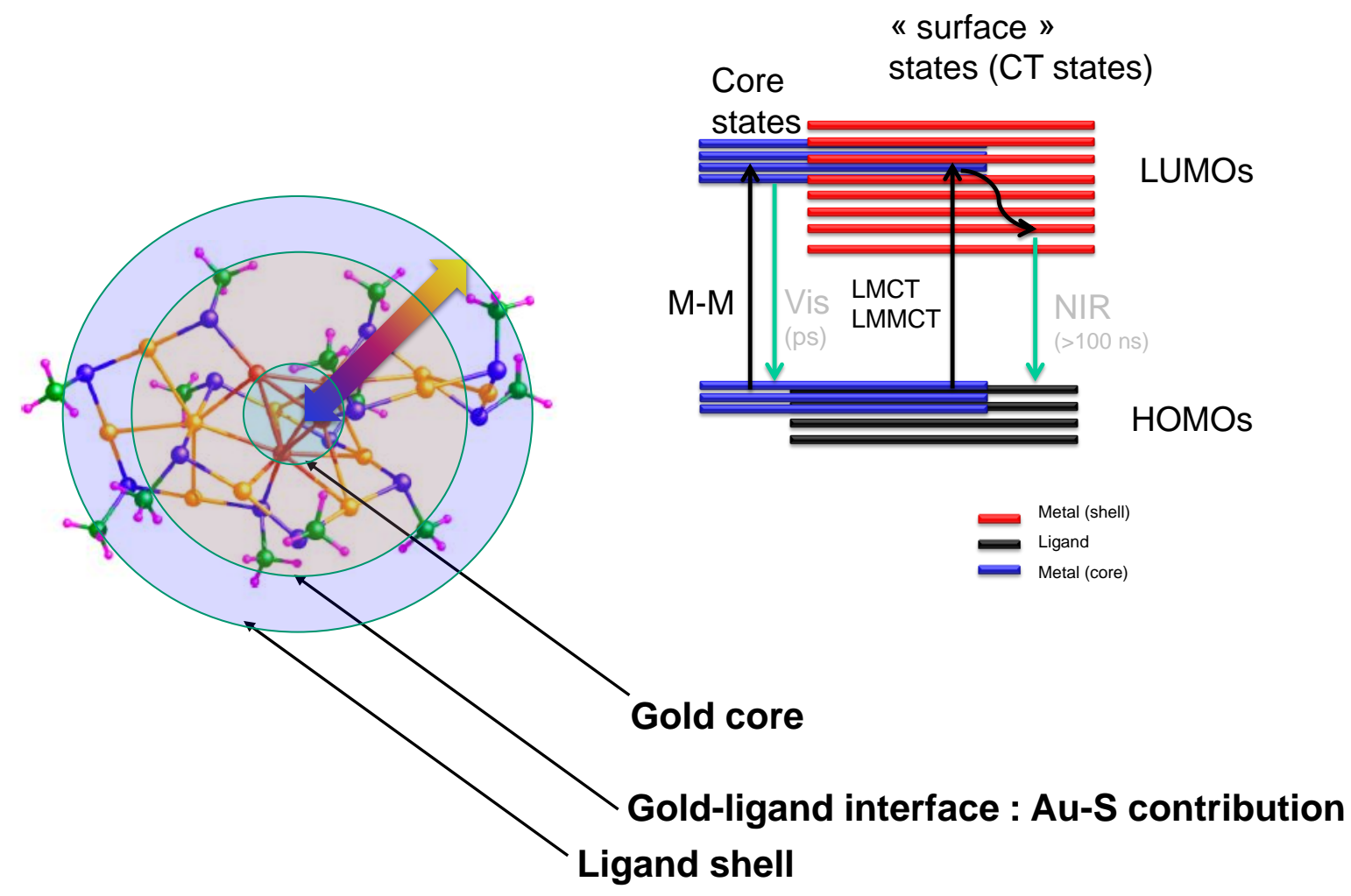

Figure 2.7 : Cartoon Diagram Showing the Relaxation Pathways in gold nanoclusters. Such nanoclusters can be viewed as a "multi-shell system" exemplified with $\mathrm{Au}_{15} \mathrm{SR}_{13}$ clusters (left).

\section{References}

[1] U. Kreibig, M. Vollmer, Optical Properties of Metal Clusters, Springer Verlag, Berlin Heidelberg, 1995.

[2] N. E. Christensen. The Band Structure of Silver and Optical Interband Transitions. physica status solidi (b) 1972, 54, 551.

[3] N. E. Christensen. Spin-orbit projected d densities-of-states of Pd, Ag, Pt, and Au. Journal of Physics F: Metal Physics 1978, 8, L51.

[4] R. Jin. Quantum sized, thiolate-protected gold nanoclusters. Nanoscale 2010, 2, 343.

[5] N. W. Ashcroft, N. Mermin, Solid State Physics Harcourt Inc. Orlando, 1976.

[6] V. Bonačić-Koutecky, V. Veyret, R. Mitrić. Ab initio study of the absorption spectra of Agn ( $n=5-8$ ) clusters. The Journal of Chemical Physics 2001, 115, 10450.

[7] F. Rabilloud. UV-visible absorption spectra of metallic clusters from TDDFT calculations. The European Physical Journal D 2013, 67, 18.

[8] R. Markus, N. Schwentner, Physics and Chemistry of Small Clusters, Plenum, New York, 1987. 
[9] W. Harbich, S. Fedrigo, J. Buttet, D. M. Lindsay. Optical spectroscopy on size selected gold clusters deposited in rare gas matrices. Zeitschrift für Physik D Atoms, Molecules and Clusters 1991, 19, 157.

[10] W. Harbich, S. Fedrigo, J. Buttet, D. M. Lindsay. Deposition of mass selected gold clusters in solid krypton. The Journal of Chemical Physics 1992, 96, 8104.

[11] B. A. Collings, K. Athanassenas, D. Lacombe, D. M. Rayner, P. A. Hackett. Optical absorption spectra of Au7, Au9, Au11, and Au13, and their cations: Gold clusters with 6, 7, 8, 9, 10, 11, 12, and 13 s-electrons. The Journal of Chemical Physics 1994, 101, 3506.

[12] U. Kreibig. Electronic properties of small silver particles: the optical constants and their temperature dependence. Journal of Physics F: Metal Physics 1974, 4, 999.

[13] W. Harbich, S. Fedrigo, F. Meyer, D. M. Lindsay, J. Lignieres, J. C. Rivoal, D. Kreisle. Deposition of mass selected silver clusters in rare gas matrices. The Journal of Chemical Physics 1990, 93, 8535.

[14] C. Sieber, J. Buttet, W. Harbich, C. Félix, R. Mitrić, V. Bonačić-Koutecký. Isomer-specific spectroscopy of metal clusters trapped in a matrix: $\left\{\{\text { mathrm }\{\mathrm{Ag}\}\}_{-}\{9\} \$\right.$. Physical Review $A$ 2004, 70, 041201.

[15] W. Chen, Z. Wang, Z. Lin, L. Lin, K. Fang, Y. Xu, M. Su, J. Lin. Photostimulated luminescence of Agl clusters in zeolite-Y. Journal of Applied Physics 1998, 83, 3811.

[16] L. A. Peyser, A. E. Vinson, A. P. Bartko, R. M. Dickson. Photoactivated Fluorescence from Individual Silver Nanoclusters. Science 2001, 291, 103.

[17] U. Hild, G. Dietrich, S. Krückeberg, M. Lindinger, K. Lützenkirchen, L. Schweikhard, C. Walther, J. Ziegler. Time-resolved photofragmentation of stored silver clusters $\mathrm{Ag}<$ sup $>+</$ sup $><$ sub $><\mathrm{i}>\mathrm{n}</ \mathrm{i}></$ sub $>(<\mathrm{i}>\mathrm{n}</ \mathrm{i}>=8-21)$. Physical Review A: Atomic, Molecular, and Optical Physics 1998, 57, 7.

[18] J. Zheng, R. M. Dickson. Individual Water-Soluble Dendrimer-Encapsulated Silver Nanodot Fluorescence. Journal of the American Chemical Society 2002, 124, 13982.

[19] C. I. Richards, S. Choi, J.-C. Hsiang, Y. Antoku, T. Vosch, A. Bongiorno, Y.-L. Tzeng, R. M. Dickson. Oligonucleotide-Stabilized Ag Nanocluster Fluorophores. Journal of the American Chemical Society 2008, 130, 5038.

[20] Z. Wu, R. Jin. On the Ligand's Role in the Fluorescence of Gold Nanoclusters. Nano Letters 2010, 10, 2568.

[21] I. Diez, R. H. A. Ras. Fluorescent silver nanoclusters. Nanoscale 2011, 3, 1963.

[22] M. Brust, M. Walker, D. Bethell, D. J. Schiffrin, R. Whyman. Synthesis of thiol-derivatised gold nanoparticles in a two-phase Liquid-Liquid system. Journal of the Chemical Society, Chemical Communications 1994, 801.

[23] Y. Negishi, K. Nobusada, T. Tsukuda. Glutathione-Protected Gold Clusters Revisited: Bridging the Gap between Gold(I)-Thiolate Complexes and Thiolate-Protected Gold Nanocrystals. Journal of the American Chemical Society 2005, 127, 5261.

[24] Y. Negishi, Y. Takasugi, S. Sato, H. Yao, K. Kimura, T. Tsukuda. Magic-Numbered Aun Clusters Protected by Glutathione Monolayers ( $\mathrm{n}=18,21,25,28,32,39)$ : Isolation and Spectroscopic Characterization. Journal of the American Chemical Society 2004, 126, 6518.

[25] T. G. Schaaff, G. Knight, M. N. Shafigullin, R. F. Borkman, R. L. Whetten. Isolation and Selected Properties of a 10.4 kDa Gold:Glutathione Cluster Compound. The Journal of Physical Chemistry B 1998, 102, 10643.

[26] C. Zeng, Y. Chen, A. Das, R. Jin. Transformation Chemistry of Gold Nanoclusters: From One Stable Size to Another. The Journal of Physical Chemistry Letters 2015, 6, 2976.

[27] R. Jin, C. Zeng, M. Zhou, Y. Chen. Atomically Precise Colloidal Metal Nanoclusters and Nanoparticles: Fundamentals and Opportunities. Chemical Reviews 2016, 116, 10346.

[28] R. Jin. Atomically precise metal nanoclusters: stable sizes and optical properties. Nanoscale 2015, 7, 1549.

[29] K. M. Harkness, D. E. Cliffel, J. A. McLean. Characterization of thiolate-protected gold nanoparticles by mass spectrometry. Analyst 2010, 135, 868. 
[30] A. Dass, A. Stevenson, G. R. Dubay, J. B. Tracy, R. W. Murray. Nanoparticle MALDI-TOF Mass Spectrometry without Fragmentation: Au25( $\mathrm{SCH} 2 \mathrm{CH} 2 \mathrm{Ph}) 18$ and Mixed Monolayer Au25(SCH2CH2Ph)18-x(L)x. Journal of the American Chemical Society 2008, 130, 5940.

[31] S. Kumar, M. D. Bolan, T. P. Bigioni. Glutathione-Stabilized Magic-Number Silver Cluster Compounds. Journal of the American Chemical Society 2010, 132, 13141.

[32] R. Hamouda, F. Bertorelle, D. Rayane, R. Antoine, M. Broyer, P. Dugourd. Glutathione capped gold $\mathrm{AuN}(\mathrm{SG}) \mathrm{M}$ clusters studied by isotope-resolved mass spectrometry. International Journal of Mass Spectrometry 2013, 335, 1.

[33] P. D. Jadzinsky, G. Calero, C. J. Ackerson, D. A. Bushnell, R. D. Kornberg. Structure of a Thiol Monolayer-Protected Gold Nanoparticle at 1.1 Å Resolution. Science 2007, 318, 430.

[34] Z. Wu, C. Gayathri, R. R. Gil, R. Jin. Probing the Structure and Charge State of GlutathioneCapped Au25(SG)18 Clusters by NMR and Mass Spectrometry. Journal of the American Chemical Society 2009, 131, 6535. 


\section{Chapter 3}

\section{A Brief Survey of Nonlinear Optics: Second-Harmonic Generation and Two- Photon Absorption}

\section{Introduction.}

The purpose of this chapter is to introduce the theoretical fundamentals responsible for nonlinear optical (NLO) processes. ${ }^{[1]}$ Fascinating key processes such as second harmonic generation (SHG), third harmonic generation (THG) and two-photon absorption (TPA) are consequences of light-matter interaction described by higher order corrections. The use of these techniques is the most popular for NLO imaging which is a powerful microscopy technique that has found growing application in fields ranging from fundamental physics to biomedicine. ${ }^{[2,3]}$ As it will be demonstrated in this book, liganded gold and silver quantum nanoclusters are promising candidates as contrast agents in nonlinear optical imaging applications, e.g. second-harmonic generation ( $\mathrm{SHG}$ ) and two-photon excited fluorescence 
(TPEF). Thus, we will focus on the general theoretical background of second harmonic generation and two-photon absorption.

\section{Nonlinear optical phenomena}

The field of optics investigates the interaction between matter and light resulting in specific optical properties of individual material which are essentially determined by the response of the electrons to an external electric field. Charges within a material are displaced under the influence of an external electric field $(\boldsymbol{E}(\boldsymbol{t}))$. In the linear dipole approximation, this displacement of charges results in an induced dipole moment per unit volume of material called the polarization $(\boldsymbol{P}(\boldsymbol{t}))$ - which is proportional to the electric field. The constant of proportionality that relates the applied electric field to the polarization is called the linear susceptibility $\left(\chi^{(1)}\right)$.

$\boldsymbol{P}(\boldsymbol{t})=\chi^{(1)} \boldsymbol{E}(\boldsymbol{t})$

However, when a material is subjected to an electric field with very high intensity, induced for instance by a laser light, its polarizability change can be driven beyond the usual regime. Therefore, the induced polarization leads to nonlinear effect and can be expressed as a Taylor series in the electric field strength

$\boldsymbol{P}(\boldsymbol{t})=\chi^{(1)} \boldsymbol{E}(t)+\frac{1}{2 !} \chi^{(2)} \boldsymbol{E}^{2}(t)+\frac{1}{3 !} \chi^{(3)} \boldsymbol{E}^{3}(t)+\cdots$

This equation introduces $\chi^{(2)}$ and $\chi^{(3)}$ known as second- and third-order nonlinear optical susceptibilities, respectively.

Vector of the electric field is composed from a static part and one or more time oscillating components and can be expressed as:

$E_{I}(t)=\sum_{\omega} E_{I}(\omega) e^{-i \omega t}$ 
where $\boldsymbol{E}_{\boldsymbol{I}}(\boldsymbol{\omega})$ represent the Fourier amplitudes of the electric field along the macroscopic axis I. Furthermore, electric field vector implies that the linear susceptibility is a second-rank tensor, the second-order nonlinear optical susceptibility is a third-rank tensor, and so on.

The form of the linear dielectric response tensor allows for establishing a simpler relation between the Fourier transforms of the polarization and the applied field,

$\boldsymbol{P}(\omega)=\chi^{(1)}(\omega) \boldsymbol{E}(\omega)$

where, $\chi^{(1)}(\omega)$, the linear susceptibility tensor is the Fourier transform of the linear dielectric response tensor $\chi^{(1)}$. The tensor relation in equation (3.4) can also be written as

$P_{I}(\omega)=\sum_{J} \chi_{I J}^{(1)}(\omega) E_{J}(\omega)$

where the subscript $I$ labels the ith Cartesian coordinate $(I=\mathrm{x}, \mathrm{y}, \mathrm{z})$ and the sum is over $J=\mathrm{x}$, y, z. The tensor $\chi^{(1)}(\omega)$ thus has nine components. It is possible to write expressions analogous to equation (3.5) in terms of Fourier transforms of the nonlinear polarization, applied fields, and the dielectric response tensor. Because the nature of the polarization field is dependent on the incident electric fields, the effects resulting from the nonlinear susceptibilities are given in shorthand notation, labeled by the appropriate order of susceptibility as well as by incident and resultant electric field frequencies. Hence the total polarization can be written as

$P_{I}\left(\omega_{1}\right)=\sum_{J} \chi_{I J}^{(1)}\left(-\omega_{1} ; \omega_{2}\right) E_{J}\left(\omega_{2}\right)+\frac{1}{2 !} \sum_{J K} \chi_{I J K}^{(2)}\left(-\omega_{1} ; \omega_{2}, \omega_{3}\right) E_{J}\left(\omega_{2}\right) E_{K}\left(\omega_{3}\right)+$ $\frac{1}{3 !} \sum_{J K L} \chi_{I J K}^{(3)}\left(-\omega_{1} ; \omega_{2}, \omega_{3}, \omega_{4}\right) E_{J}\left(\omega_{2}\right) E_{K}\left(\omega_{3}\right) E_{L}\left(\omega_{4}\right)+\cdots$

where indices $I, J, K, L$ denote cartesian components of electric fields oscillating at $\omega_{1}, \omega_{2}, \omega_{3}$ and $\omega_{4}$ respectively. We are able to identify these factors for some common nonlinear optical processes, see Table 1. $\chi^{(1)}$ is the linear susceptibility tensor representing effects such as linear absorption and refraction, $\chi^{(2)}$ is the second-order nonlinear optical susceptibility, and $\chi^{(3)}$ is the third-order nonlinear susceptibility. SHG is a second-order process, whereas, TPEF and THG are both third-order processes. 
Table 1: Some important processes involving Nonlinear Optical Spectroscopy

\begin{tabular}{|ccl|}
\hline Nonlinear Optical Process & Order & Frequencies \\
\hline Linear Response & $\mathbf{1}$ & $-\omega ; \omega$ \\
\hline Pockels Effect (EO effect) & $\mathbf{2}$ & $-\omega ; \omega, 0$ \\
\hline Second Harmonic Generation (SHG) & $\mathbf{2}$ & $-2 \omega ; \omega, \omega$ \\
\hline Optical Rectification (OR) & $\mathbf{2}$ & $0 ; \omega,-\omega$ \\
\hline Intensity- dependent refractive index & $\mathbf{3}$ & $-\omega ; \omega,-\omega, \omega$ \\
\hline Dc Kerr Effect & $\mathbf{3}$ & $-\omega ; 0,0, \omega$ \\
\hline Third Harmonic Generation (THG) & $\mathbf{3}$ & $-3 \omega ; \omega, \omega, \omega$ \\
\hline degenerate Two-Photon Absorption (TPA) & $\mathbf{3}$ & $-\omega ; \omega, \omega,-\omega$ \\
\hline
\end{tabular}

More specifically, in second harmonic generation, a pump wave with a frequency of $\omega$ creates a signal at the frequency $2 \omega$. A polarization oscillating at frequency $2 \omega$ radiates an electromagnetic wave of the same frequency, which propagates with the same velocity as that of the incident pump wave. The phenomenon, where the wave produced has the same characteristics of directionality and monochromaticity as the incident wave and is emitted in the same direction, is known as the SHG (second harmonic generation).

A paramount condition in second order nonlinear optical materials is a requirement for their non-centrosymmetry. This can be illustrated by considering an electric field $\boldsymbol{E}(t)=\boldsymbol{E}_{\omega} \cos (\omega t)$ incident on a centrosymmetric medium. The resultant second order polarization for this medium is $\boldsymbol{P}(\boldsymbol{t})=\frac{1}{2 !} \chi^{(2)} \boldsymbol{E}^{2}(t)$.

If the material structure is centrosymmetric it must possess inversion symmetry, where 


$$
\begin{aligned}
& -\boldsymbol{P}(\boldsymbol{t})=\frac{1}{2 !} \chi^{(2)}(-\boldsymbol{E}(t))^{2} \text { and } \\
& \boldsymbol{P}(\boldsymbol{t})=\frac{1}{2 !} \chi^{(2)}(\boldsymbol{E}(t))^{2}
\end{aligned}
$$

Equations (3.7) hold simultaneously only when the polarization field is zero, indicating $\chi^{(2)}=0$ for centrosymmetric media.

\section{From macroscopic to microscopic nonlinear susceptibilities}

For an ensemble of $N$ molecules, the second order nonlinear susceptibility $\chi^{(2)}$ can be expressed as an orientational average over the molecules and their hyperpolarizability $\beta_{i j k} \cdot{ }^{[4]}$ Similar to equation (3.6), the microscopic polarization $\left(p_{i}\right)$ can then be expressed as

$$
\begin{aligned}
& p_{i}\left(\omega_{1}\right)=\sum_{i} \alpha_{i j}\left(-\omega_{1} ; \omega_{2}\right) E_{j}\left(\omega_{2}\right)+\frac{1}{2 !} \sum_{j k} \beta_{i j k}\left(-\omega_{1} ; \omega_{2}, \omega_{3}\right) E_{J j}\left(\omega_{2}\right) E_{k}\left(\omega_{3}\right)+ \\
& \frac{1}{3 !} \sum_{j k l} \gamma_{i j k}\left(-\omega_{1} ; \omega_{2}, \omega_{3}, \omega_{4}\right) E_{j}\left(\omega_{2}\right) E_{k}\left(\omega_{3}\right) E_{l}\left(\omega_{4}\right)+\cdots
\end{aligned}
$$

This equation introduces the linear electric polarizability $\alpha$, the first-order (nonlinear) hyperpolarizability $\beta$, the second-order hyperpolarizability $\gamma$, etc. In the literature, the polarizabilities are commonly reported in Gaussian electrostatic units (esu units) with length in $\mathrm{cm}$ and charge in statcoulomb ( $1 \mathrm{statC}=4 \pi c C$, where $c$ is the speed of light expressed in $\mathrm{ms}^{-1}$ ), whereas many theoretical studies use atomic units (a.u., with $\mathrm{e}=\hbar=\mathrm{m}_{\mathrm{e}}=1$ ).

The common convention of referring to the esu units implies the following units for $\alpha\left(\mathrm{cm}^{3}\right)$, $\beta\left(\mathrm{cm}^{5} \mathrm{statC}^{-1}\right)$ and $\gamma\left(\mathrm{cm}^{7} \mathrm{statC}^{-2}\right)$. Typical linear electric polarizability values are of the order of $10^{-24}$ esu. Values of the first-order (nonlinear) hyperpolarizability $\beta$ are of the order of $10^{-30}$ esu and the second-order hyperpolarizability $\gamma$ values are of the order of $10^{-36}$ esu.

Polarizability and the hyperpolarizabilities $\alpha, \beta$ and $\gamma$ are second-rank, third-rank, and fourthtensors, and as result, they have 9, 27, and 81 components. 
It is often convenient to describe the geometry of a system in terms of its spatial symmetry. All materials possess various spatial symmetries including rotations, reflections, and inversions about axes, points, and planes. Spatial symmetries reduce the number of independent non-zero elements in the susceptibility tensor. For example, a molecule that has $\mathrm{C}_{2 \mathrm{~V}}$ symmetry has only a few nonzero elements in the hyperpolarizability tensor, and those are $\beta_{z z z}, \beta_{z x x}$, and $\beta_{x z x}=\beta_{x x z}{ }^{[5]}$ The hyperpolarizability tensor described above is in the molecular frame. To obtain the hyperpolarizability tensor in the laboratory frame $(\mathrm{X}, \mathrm{Y}, \mathrm{Z})$, one needs to use the following expression

$\beta_{I J K}=\sum_{i j k} T_{I i} T_{J j} T_{K k} \beta_{i j k}$

where $T$ is the transformation matrix corresponding to Euler angles.

\section{Third Order Nonlinear Process: Two photon absorption}

Two photon absorption (TPA) is a third order NLO process. It corresponds to the simultaneous absorption of two photons of either the same energies (degenerate TPA) or different energies (non-degenerate TPA), in the presence of intense radiation. TPA corresponds to the third-order polarization $\chi^{(3)}(-\omega 1 ;-\omega 2, \omega 2, \omega 1)$. At sufficiently high intensities, the probability that material absorbs more than one photon before relaxing to the ground state can be substantially increased. Two-photon absorption involves a transition from the ground state of a system to a higher-lying excited state by the simultaneous absorption of two photons from an incident radiation field.

In order to define the TPA cross section, one has to consider the average change of absorbed energy per volume unit when subjected to an external electric field, $\boldsymbol{E}$, and can be written $\mathrm{as}^{[6]}$ 
$\left\langle\frac{d}{d t}\left(\frac{\text { absorbed energy }}{\text { volume }}\right)\right\rangle_{\text {time }}=\langle\boldsymbol{j} \cdot \boldsymbol{E}\rangle$

The current density, $\boldsymbol{j}$, induced in the medium can be expanded as in the electric dipole approximation $\boldsymbol{j}=\frac{\partial \boldsymbol{P}}{\partial \boldsymbol{t}}$. The rate of absorbed energy can thus be written as

$\left\langle\frac{\mathrm{d}}{\mathrm{dt}}\left(\frac{\text { absorbed energy }}{\text { volume }}\right)\right\rangle_{\text {time }}=\langle\mathrm{j} \cdot \mathrm{E}\rangle=\left\langle\frac{\partial \mathrm{P}^{(1)}}{\partial \mathrm{t}} \cdot \mathrm{E}\right\rangle+\left\langle\frac{\partial \mathrm{P}^{(3)}}{\partial \mathrm{t}} \cdot \mathrm{E}\right\rangle+\cdots$

where it is sufficient to consider terms of odd order, because contributions from terms of even order will cancel out in the time averaging procedure. ${ }^{[7]}$

The second term corresponds to the two-photon absorption. Near a resonance, the susceptibility tensor becomes complex, and the imaginary part describes absorption (or gain) properties. The two-photon absorption coefficient is proportional to the imaginary part of the third-order susceptibility tensor and can be identified as

$\left\langle\frac{\partial \boldsymbol{P}^{(3)}}{\partial \boldsymbol{t}} \cdot \boldsymbol{E}\right\rangle=\frac{3}{8} \omega \operatorname{Im}\left[\chi_{I J}^{(3)}(-\omega ; \omega,-\omega, \omega)\right] E_{1, I} E_{1, J}^{*} E_{1, K} E_{1, K}^{*}$

The expressions can be abbreviated further by introducing the optical intensity, $I$, as $I=$ $\frac{n c}{8 \pi} E^{2}$, where $\mathrm{n}$ is the index of refraction of the medium and $\mathrm{c}$ the speed of light in vacuum, the energy absorbed through two-photon processes is, therefore

$\left\langle\frac{d}{d t}\left(\frac{\text { absorbed energy }}{\text { volume }}\right)\right\rangle_{\text {time }}=\frac{24 \pi^{2} \omega}{n^{2} c^{2}} \operatorname{Im}\left[\chi_{I J}^{(3)}(-\omega ; \omega,-\omega, \omega)\right]$

The TPA activity is usually quantified through the so called two-photon absorption cross section $\sigma_{2}(\omega)$ defined by the rate equation

$\frac{d n_{p}}{d t}=\sigma_{2}(\omega) N F^{2}$

where $\frac{d n_{p}}{d t}$ is the number of photons absorbed per unit time through a TPA process, $\mathrm{N}$ is the density of absorbing species and $F=\frac{I}{\hbar \omega}$ being the photon flux. Since $d W=d n_{p} \hbar \omega$,

$\sigma_{2}(\omega)=\frac{24 \pi^{2} \hbar \omega^{2}}{n^{2} c^{2} N} \operatorname{Im}\left[\chi_{I J}^{(3)}(-\omega ; \omega,-\omega, \omega)\right]$ 
Notice that constants and corresponding numerical factors might differ in eq. 3.15. The definition we adopt here is, however, the most widely used and accepted.

\section{Parametric vs non-parametric processes}

The generation of harmonics is a parametric process described by a real susceptibility. It differs significantly from non-parametric processes, such as multiphoton absorption, which have a complex susceptibility associated with them (see equation (3.15). For parametric processes, the initial and final quantum-mechanical states are identical, as shown in Fig. 3.1 panels b) and c). In between these states, the population can temporally reside in a "virtual" state represented by dashed horizontal lines in Fig. 3.1. Since parametric processes conserve photon energy, no energy is deposited into the system. In non-parametric processes, the initial and final states are different as represented in Fig. 3.1 panel a), thus there is a net population transfer from one real level to another. Note that non-parametric interactions lead to photon absorption that, due to population transfer, may induce thermal damage.

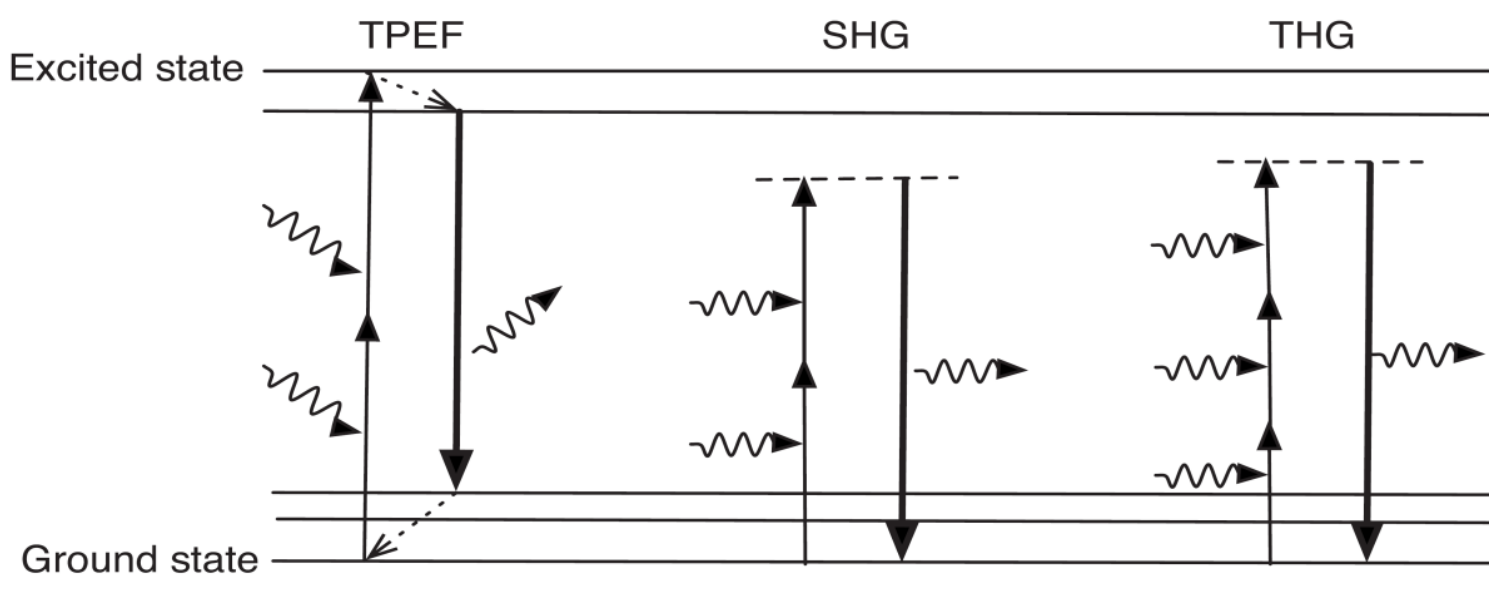

(a)

(b)

(c)

Figure 3.1 : Schematic representation of nonlinear processes: a) TPA followed by TPEF , b) SHG, c) THG. Wiggly lines represent incoming and radiated photons, dashed lines represent virtual states, and dashed arrows nonradiative relaxation processes. 


\section{References.}

[1] R. W. Boyd, Nonlinear Optics, Second Edition, 2003; and Third Edition 2008 ed., Academic Press, Boston, 1992.

[2] P. J. Campagnola, L. M. Loew. Second-harmonic imaging microscopy for visualizing biomolecular arrays in cells, tissues and organisms. Nature Biotechnology 2003, 21, 1356.

[3] R. Carriles, D. N. Schafer, K. E. Sheetz, J. J. Field, R. Cisek, V. Barzda, A. W. Sylvester, J. A. Squier. Invited Review Article: Imaging techniques for harmonic and multiphoton absorption fluorescence microscopy. Review of Scientific Instruments 2009, 80, 081101.

[4] D. R. Kanis, M. A. Ratner, T. J. Marks. Design and construction of molecular assemblies with large second-order optical nonlinearities. Quantum chemical aspects. Chemical Reviews 1994, 94, 195.

[5] P. C. Ray. Size and Shape Dependent Second Order Nonlinear Optical Properties of Nanomaterials and Their Application in Biological and Chemical Sensing. Chemical Reviews 2010, 110, 5332.

[6] F. Terenziani, C. Katan, E. Badaeva, S. Tretiak, M. Blanchard-Desce. Enhanced Two-Photon Absorption of Organic Chromophores: Theoretical and Experimental Assessments. Advanced Materials 2008, 20, 4641.

[7] H. Mahr., Quantum Electronics, Academic Press, New York, 1975. 


\section{Chapter 4}

\section{Computational Evaluation of Optical Nonlinearities}

\section{Quantum chemical approaches}

Significant advancement of reliable computational techniques for calculations of nonlinear optical properties of molecules has been achieved in the last two decades. In particularly this is the case for calculations of the first and second order hyperpolarizablities implemented in numerous quantum-chemical packages. Resonant properties such as two-photon and threephoton absorption have been implemented in only few of them. The inclusion of the influence of the environment on NLO properties has been also achieved. For evaluation of nonlinear response of molecular systems, two different approaches can be employed: the analytic response theory which is more rigorous and sum-over-states (SOS) methods, both based on perturbation theory. Within these approaches the various methods with different quality of accuracy of electronic excited states can be used.

Merging success of density functional theory in chemistry with analytic time dependent response theory, ${ }^{[1,2,3]}$ SOS approach ${ }^{[4]}$ or combination of both provides reliable tool for the general use. Foundation of time dependent $\mathrm{DFT}^{[5]}$ has stimulated numerous studies and applications involving time dependent response properties. First linear properties such as 
excitation energies and polarizabilities have been studied and later on quadratic and third order response relevant for nonlinear optics have been developed. Analytic response theory based on time dependent perturbation theory provides response of the system to different perturbations (dynamic, static, internal and external). Formulation of TDDFT beyond localdensity approximation within analytic response approach allowing for calculation of static and frequency-dependent nonlinear properties is of particular interest for applications. In this context, the two-photon absorption TPA cross sections gained particular attention since they depend quadratically on the intensity of the excited light and the excitations can be reached by applying half of the wavelengths. This allows to increase the penetrability of materials and tissue and therefore it is attractive for applications in the area of optical materials and in medicine within singlet-oxygen therapy and fluorescence marking.

There are two approaches for addressing two-photon absorption within analytic response method: First, the calculation of third-order frequency-dependent response function from the second hyperpolarizability in which imaginary part is related to TPA cross section; Second involves the single residue of the second-order response function or the first hyperpolarizability. The latter represents more practical way of computing the TPA cross section and will be briefly outlined below. In spite of the success, it is necessary to notice that in standard response theory, the response functions can diverge, since the response function has poles whenever one or more of the optical frequencies equal an excitation energy. This can lead to nonphysical behavior for molecular properties in the resonance region. However, introducing damping terms in different ways the singularities of the response functions can be corrected or effectively removed.

The second more approximate method commonly used for two-photon absorptions is conventional sum-over-states technique. Since SOS formulae express the nonlinear optical properties in terms of energies and dipole moments of excited states, transition moments 
between various excited states, the NLO properties are usually interpreted in terms of these observables. In general, the summation of excited states converges very slowly. However, it has been shown that the molecules with low lying excited states of charge transfer character represent exceptions. Therefore SOS method serves as the basis for so-called few-states models which are less rigorous approaches. However these models require the calculations of transition dipole moments among the manifold of excited states which is computationally demanding.

If necessary, the combination of both methods, analytic response theory and the SOS, can be useful. In those cases the SOS can be applied to correct the divergence of response theory by introducing damping factors. Alternatively, damped response approach might be applied.

Since theoretical approach could contribute to propose concept for designing systems with large nonlinear properties e.g. TPA cross sections which should stimulate their experimental realization, we start with definition connecting measurements with theory.

The two-photon cross section for excitation from the ground state $|0\rangle$ to the final state $|f\rangle$ corresponding to experimental measurements is defined as:

$$
\delta_{T P A}=\frac{(2 \pi e)^{4} \omega_{v} \omega_{\mu}}{c^{2}} g\left(\omega_{\nu}+\omega_{\mu}\right)\left|T^{\omega_{\nu} \omega_{\mu} f}\right|^{2}
$$

where $g\left(\omega_{v}+\omega_{\mu}\right)$ is normalized line-shaped function and $T^{\omega_{\nu} \omega_{\mu} f}$ is the two-photon absorption amplitude tensor. Experimental measurement produces average TPA cross section which can be evaluated by averaging the two-photon transition amplitude tensor $\left|T^{\omega_{\nu} \omega_{\mu}, f}\right|^{2}$ over all orientations. Cartesian components $T_{a b}^{2 \omega, f}$ between the ground state $|0\rangle$ and the excited state $|f\rangle$ are defined as:

$$
T_{a b}^{2 \omega, f}=\sum_{k>0}\left[\frac{\left\langle 0\left|\hat{\mu}_{a}\right| k\right\rangle\left\langle k\left|\hat{\mu}_{b}\right| f\right\rangle}{\omega_{k}-\omega_{f} / 2}+\frac{\left\langle 0\left|\hat{\mu}_{b}\right| k\right\rangle\left\langle k\left|\hat{\mu}_{a}\right| f\right\rangle}{\omega_{k}-\omega_{f} / 2}\right](a, b=x, y, z)
$$


where frequency of incoming radiation is equal to half of this excitation energy from the ground state $|0\rangle$ to the excited state $|f\rangle$, with $\omega_{k}=\omega_{f} / 2$. The quantities $\mu_{a}$ and $\mu_{b}$ are the Cartesian components of the dipole moments operator $\hat{\mu}$ and $\omega_{k}$ and $\omega_{f}$ are the frequencies of the excitation from $|0\rangle$ to $|k\rangle$ and $|f\rangle$, respectively.

The application of this formula has limitations because it requires both summation over excited states of the system and computation of matrix elements of dipole moment operator $\hat{\mu}$ between excited states. In order to simplify the procedure, "few states model" became attractive in which dominating terms are considered, but the calculations nevertheless involve for the components mentioned above within adequate quantum chemical methods.

More rigorous way to evaluate TPA transition amplitudes tensor $T^{2 \omega, f}$ is to use response theory in which instead of summation over excited states a set of coupled equations have to be solved. In this way the necessary information about excited states can be determined by time evaluation of wave function or the electron density. In particularly in the latter case, the calculation of TPA transition amplitude tensors can be determined for larger molecules and systems and has been implemented in number of quantum chemical packages.

The environmental effects such as influence of solvent on nonlinear properties with polarizable continuum model have been also included into response theory. For instance, a set of prototypical two-photon chromophores (i.e. pure $\pi$ system, and its substituted homologs obtained employing a donor and an acceptor groups) have been selected to probe solvent effects. The results show a significant solvent dependence of the TPA cross section and anunusual trend when passing from cyclohexane to water. ${ }^{[6]}$

\section{Response Theory}


If the molecule or system is in the presence of a time-dependent external field which oscillates with a given frequency, the observables starts to oscillate and becomes time dependent. The response theory describes interaction between the molecular system and external field. The response of observable can be expanded in the powers of the field strength: Linear response, Quadratic response etc. ${ }^{[7,8]}$

For this purpose $\widehat{H}$ is total Hamiltonian operator

$$
\widehat{\mathrm{H}}=\widehat{\mathrm{H}}_{0}+\widehat{\mathrm{V}}^{t}
$$

with time independent part $\widehat{\mathrm{H}}_{0}$ satisfying time-independent Schrödinger equation and $\widehat{\mathrm{V}}^{t}$ is time-dependent perturbation. Therefore

$$
\widehat{H}|\overline{0}(t)\rangle=i \frac{\partial}{\partial t}|\overline{0}(t)\rangle
$$

needs to be considered. $\widehat{\mathrm{V}}^{t}$ is Hermitian and periodic. In frequency domain can be written:

$$
\widehat{V}^{t}=\sum_{j=-N}^{N} \exp \left(-i \omega_{j} t\right) \widehat{V}^{\omega_{j}} ; \quad \widehat{V}^{\omega_{j}}=\sum_{B} \varepsilon_{B}\left(\omega_{j}\right) B
$$

where $\varepsilon_{B}\left(\omega_{j}\right)$ is perturbation strength parameter for the operator $B$ at frequency $\omega_{j}$ which controls the field amplitude.

The compact form can be written as:

$$
\hat{V}^{t}=\sum_{b} \exp \left(-i \omega_{b} t\right) \varepsilon_{b} B
$$

Index $b$ combines frequency (j) and the operator (B) indices. $\widehat{\mathrm{V}}^{t}$ has to be Hermitian.

The wave function $|\overline{0}\rangle$ in the phase-isolated form can be written:

$$
|\overline{0}\rangle=\exp [-i F(t)]|\tilde{0}\rangle,
$$

where $|\tilde{0}\rangle$ takes form: 


$$
|\tilde{0}\rangle=|0\rangle+\left|\tilde{0}^{(1)}(t)\right\rangle+\left|\tilde{0}^{(2)}(t)\right\rangle+\cdots
$$

and is normalized.

When time-dependent perturbation is applied, the energy is not eigenvalue of $\widehat{H}$, but quasienergy $\mathrm{Q}(\mathrm{t})$ can be introduced as the time derivative of the phase function $F(t)$. Since $\widehat{H}$ commutes with $\exp [-i F(t)]$, the quasi-energy is:

$$
Q(t)=\dot{F}(t)=\left\langle\tilde{0}\left|\widehat{H}-i \frac{\partial}{\partial t}\right| \tilde{0}\right\rangle
$$

It can be seen that $Q(t) \rightarrow E_{0}$ for the unperturbed system.

The observable of a molecule in the presence of external filed can be expanded in the powers of the filed strength:

$$
\begin{aligned}
& A_{A v}(t)=\langle\overline{0}(t)|A| \overline{0}(t)\rangle=\langle\tilde{0}(t)|A| \tilde{0}(t)\rangle \\
& =\langle 0|A| 0\rangle+\sum_{b} \exp \left(-\omega_{b} t\right)\langle\langle A ; B\rangle\rangle_{\omega_{b}} \varepsilon_{b} \\
& +\frac{1}{2} \sum_{b, c} \exp \left[-i\left(\omega_{b}+\omega_{c}\right) t\right]\langle\langle A ; B, C\rangle\rangle_{\omega_{b,} \omega_{c}} \varepsilon_{b} \varepsilon_{c} \\
& +\frac{1}{6} \sum_{b, c, d} \exp \left[-i\left(\omega_{b}+\omega_{c}+\omega_{d}\right) t\right]\langle\langle A ; B, C, D\rangle\rangle_{\omega_{b}, \omega_{c}, \omega_{d}} \varepsilon_{b} \varepsilon_{c} \varepsilon_{d} \\
& +\cdots
\end{aligned}
$$

The function $\langle\langle A, B\rangle\rangle_{\omega_{b}}$ is linear response function, while $\langle\langle A, B, C\rangle\rangle_{\omega_{b}, \omega_{c}}$ and $\langle\langle A, B, C, D\rangle\rangle_{\omega_{b}, \omega_{c}, \omega_{d}}$ quadratic and cubic response functions, respectively.

\section{The interpretation of the response functions}

The linear response function can be written in the form:

$$
\langle\langle A ; B\rangle\rangle_{\omega}=\sum_{n}\left(\frac{A^{0 n} B^{n 0}(\omega)}{\omega-\omega_{n}}-\frac{B^{0 n}(\omega) A^{n 0}}{\omega+\omega_{n}}\right),
$$


where $A^{n m}=\langle n|A| m\rangle$ is a transition matrix element of the operator A between states $|n\rangle$ and $|m\rangle$. The response of the molecule with a dipole moment $\mu$, on an external homogeneous electric field oscillating with frequency $\omega_{b}$ has been obtained from the expansion in Eq. (4.4.10), where the operator $A$ is replaced by the electric dipole operator. The $\left(\mu_{\alpha}, \mu_{\beta}\right)$ th component of the linear response function gives the $\alpha \beta$ component of the frequencydependent linear polarizability tensor:

$$
\alpha_{\alpha \beta}=-\left\langle\left\langle\mu_{\alpha} ; \mu_{\beta}\right\rangle\right\rangle_{\omega_{b}}=-\sum_{m}\left(\frac{\mu_{\alpha}^{0 m} \mu_{\beta}^{m 0}}{\omega_{b}-\omega_{m}}-\frac{\mu_{\beta}^{0 m} \mu_{\alpha}^{m 0}}{\omega_{b}+\omega_{m}}\right)
$$

which describes the absorption of one photon of energy $\omega_{b}$ and the emission of one photon of energy $\omega_{b}$. The residues corresponding to the poles at $\omega_{b}= \pm \omega_{f}$ of Eq. (4.4.12),

$$
\begin{gathered}
\lim _{\omega_{b} \rightarrow \omega_{f}}\left(\omega_{b}-\omega_{f}\right)\left\langle\left\langle\mu_{\alpha} ; \mu_{\beta}\right\rangle\right\rangle_{\omega_{b}}=\mu_{\alpha}^{0 f} \mu_{\beta}^{f 0} \\
\lim _{\omega_{b} \rightarrow-\omega_{f}}\left(\omega_{b}+\omega_{f}\right)\left\langle\left\langle\mu_{\alpha} ; \mu_{\beta}\right\rangle\right\rangle_{\omega_{b}}=-\mu_{\alpha}^{f 0} \mu_{\beta}^{0 f}
\end{gathered}
$$

provide information about the dipole transition matrix element between the referent state $|0\rangle$ and $|f\rangle$. The quadratic response function can be written in the form:

$$
\begin{aligned}
& \langle\langle A ; B, C\rangle\rangle_{\omega_{b}, \omega_{c}}= \\
& =\frac{1}{2} \sum_{m, n}\left(\frac{A^{0 n} B^{n m} C^{m 0}}{\left(\omega_{b}+\omega_{c}-\omega_{n}\right)\left(\omega_{c}-\omega_{m}\right)}+\frac{A^{0 n} C^{n m} B^{m 0}}{\left(\omega_{b}+\omega_{c}-\omega_{n}\right)\left(\omega_{b}-\omega_{m}\right)}\right. \\
& +\frac{C^{0 n} B^{n m} A^{m 0}}{\left(\omega_{b}+\omega_{c}+\omega_{n}\right)\left(\omega_{c}+\omega_{m}\right)}+\frac{B^{0 n} C^{n m} A^{m 0}}{\left(\omega_{b}+\omega_{c}+\omega_{n}\right)\left(\omega_{b}+\omega_{m}\right)} \\
& \left.+\frac{B^{0 n} A^{n m} C^{m 0}}{\left(\omega_{b}+\omega_{n}\right)\left(\omega_{c}-\omega_{m}\right)}+\frac{C^{0 n} A^{n m} B^{m 0}}{\left(\omega_{c}+\omega_{n}\right)\left(\omega_{b}-\omega_{m}\right)}\right)
\end{aligned}
$$

and can be obtained from Eq. (4.4.15) when a homogeneous electric external fields of frequencies $\omega_{b}$ and $\omega_{c}$ are applied on the molecule with the dipole moment $\mu$. The operators $A, B$ and $C$ are components of the electric dipole operator. The $\left(\mu_{\alpha}, \mu_{\beta}, \mu_{\gamma}\right)$-th component of the quadratic response function is identical to the (minus) $(\alpha \beta \gamma)$ th component of the electric 
frequency-dependent dipole hyperpolarizability tensor ${ }^{[9]}$ at frequencies $\omega_{b}, \omega_{c}$. The frequencydependent hyperpolarizability describes the absorption of two photons, one of frequency $\omega_{b}$ and one of frequency $\omega_{c}$, and the emission of one photon of frequency $\omega=\omega_{b}+\omega_{c}$.

Notice that the polarization can be expressed as a Taylor's series in the electric filed strength:

$$
\mu(t)=\mu^{0}+\alpha E(t)+\frac{1}{2} \beta E^{2}(t)+\frac{1}{6} \gamma E^{3}(t)+\cdots
$$

where $\mu^{0}$ is permanent dipole moment of the molecule, $\alpha$ is linear electric polarizability, $\beta$ is the first order nonlinear hyperpolarizability and $\gamma$ is the second order hyperpolarizability. Since linear and nonlinear polarizability depend on the frequency of applied filed, time dependent polarization can have frequency components separated from those of external field due to power dependence on the electric field strength. Therefore, the system can emit sumfrequency generated radiation. Eq. (4.4.16) shows the fundamental origin of nonlinearities on microscopic level through the expansion coefficients in this equation in which hyperpolarizabilities are responsible for nonlinear optical performance of the material. In this context theoretical modeling of nonlinear optical properties determines these quantities taking into account the structure of the system.

In the Table 4.1, we summarized molecular properties that can be obtained from the linear and nonlinear response functions.

Table 4.1 Molecular properties described by the first-, second-, and third-order response functions, compare table from reference ${ }^{[7]}$

\begin{tabular}{cll}
\hline Response & Residue & Molecular property \\
\hline $\boldsymbol{\alpha}(-\boldsymbol{\omega} ; \boldsymbol{\omega})$ & Linear electric dipole polarizability \\
& & One-photon transition matrix elements between the \\
& & ground state $|0\rangle$ and the excited state $|\mathrm{f}\rangle$. \\
\hline $\boldsymbol{\beta}\left(-\boldsymbol{\omega} ; \boldsymbol{\omega}_{\boldsymbol{b}}, \boldsymbol{\omega}_{\boldsymbol{c}}\right)$ & First-order & nonlinear electric dipole \\
\hline
\end{tabular}




\begin{tabular}{|c|c|c|}
\hline & & hyperpolarizability. \\
\hline & $\omega_{c}=\omega_{f 0}$ & $\begin{array}{l}\text { Two-photon transition matrix element between the } \\
\text { ground state }|0\rangle \text { and the excited state }|\mathrm{f}\rangle \text {. }\end{array}$ \\
\hline & $\omega_{b}=-\omega_{f 0}$ & One-photon transition matrix element between the \\
\hline & $\omega_{c}=\omega_{g 0}$ & excited states $|f\rangle$ and $|g\rangle$. \\
\hline & $\omega_{b}=-\omega_{f 0}$ & Permanent electric dipole moment of the excited \\
\hline & $\omega_{c}=\omega_{f 0}$ & state $|\mathrm{f}\rangle$ \\
\hline \multirow{8}{*}{$\gamma\left(-\omega ; \omega_{b}, \omega_{c}, \omega_{d}\right)$} & & Second-order nonlinear electric dipole \\
\hline & & hyperpolarizability \\
\hline & & Three-photon transition matrix element between the \\
\hline & $\omega_{d}=\omega_{f 0}$ & ground state $|0\rangle$ and the excited state $|\mathrm{f}\rangle$. \\
\hline & $\omega_{c}=-\omega_{f 0}$ & Two-photon transition matrix element between the \\
\hline & $\omega_{d}=\omega_{g 0}$ & excited states $|\mathrm{f}\rangle$ and $|\mathrm{g}\rangle$. \\
\hline & $\omega_{c}=-\omega_{f 0}$ & Linear electric dipole polarizability of the excited \\
\hline & $\omega_{d}=\omega_{f 0}$ & state $|\mathrm{f}\rangle$. \\
\hline
\end{tabular}

In response theory the TPA transition amplitude can be obtained from a single residue of a quadratic response function $\langle\langle A, B, C\rangle\rangle_{\omega_{b}, \omega_{c}}$ corresponding to multiplication of the first order $\boldsymbol{T}^{C, f}$ and the second order transition amplitude $\boldsymbol{T}^{A B, f}$ between the ground state| 0 >and excited state $|f\rangle$ :

$$
\operatorname{Res}\langle\langle A ; B, C\rangle\rangle_{\omega_{B}, \omega_{C}}=\lim _{\omega_{C} \rightarrow \omega_{f}}\left(\omega_{C}-\omega_{f}\right)\langle\langle A ; B, C\rangle\rangle_{\omega_{B}, \omega_{C}}=-T^{A B, f} T^{C, f}
$$

Replacing operators $A, B$ and $C$ by the Cartesian components of dipole moment operator $\mu$ and setting frequency of the time-dependent perturbation $B$ to satisfy condition $\omega_{b}=\omega_{f} / 2$, the single residue of quadratic response function can be rewritten as:

$$
\lim _{\omega_{C} \rightarrow \omega_{f}}\left(\omega_{C}-\omega_{f}\right)\left\langle\left\langle\hat{\mu}_{a} ; \hat{\mu}_{b}, \hat{\mu}_{c}\right\rangle \frac{\omega_{f}}{2}, \omega_{C}=-T_{a b}^{2 \omega, f}\left\langle f\left|\hat{\mu}_{C}\right| 0\right\rangle\right.
$$


The right-hand side of equation (4.18) corresponds to the product of Cartesian tensor element and one-photon absorption Cartesian vector element $\left\langle f\left|\hat{\mu}_{C}\right| 0\right\rangle$. Therefore it can be used to obtain $T_{a b}^{2 \omega, f}$ from the single residue of the quadratic response function. ${ }^{[10,11,12]}$ Using spectral representation it can be shown that $T_{a b}^{2 \omega, f}$ determined from equation (4.4.18) is equivalent to the TPA amplitude tensor from equation (4.4.1), and therefore connecting theoretical and experimental observables.

In order to obtain the TPA amplitude tensor, one needs to solve a set of response equations which determine the single residue of $\left\langle\left\langle\hat{\mu}_{a} ; \hat{\mu}_{b}, \hat{\mu}_{c}\right\rangle\right\rangle_{\frac{\omega_{f}}{2}, \omega_{c}}$. For this purpose, the description of the quadratic response equations for evaluation of TPA tensors together with the numerical procedures for their solution, are implemented in different quantum chemistry packages. Quadratic response-DFT approach is the mostly used.

\section{Corrections of singularities in the quadratic response}

a)The damped response theory. ${ }^{[13]}$ As already mentioned, standard response functions have singularities when one or more of optical frequencies equal the excitation energies which leads to divergences when solving the response equations and consequently unphysical behavior for molecular properties. In order to obtain the corrected physical behavior at the resonance frequencies, different approaches have been developed. One of them is damped response theory in which empirical damping terms are introduced by extending the domain of response function to complex domain. The damped response functions effectively remove singularities and therefore they are well defined in entire frequency domain. In standard response theory the TPA amplitude is formally determined from residue of the quadratic response function. Since the physical observable is proportional to TPA strengths (corresponding to the amplitude squared), it equals the residua of the cubic response function, 
therefore in order to calculate damped TPA spectra it is necessary to consider damped cubic response theory.

If $|\mathrm{f}\rangle$ is higher lying excited state it may exist intermediate state $|\mathrm{k}\rangle$ half way between the ground state $|0\rangle$ and $|\mathrm{f}\rangle$. If the laser frequency is chosen to be $\omega_{k}=\omega_{f} / 2$ the one photon transitions $|0\rangle \rightarrow|\mathrm{k}\rangle$ and $|\mathrm{k}\rangle \rightarrow|\mathrm{f}\rangle$ will be simultaneously resonant corresponding to so called double resonance situation. Thus, in this case the k-th component of the expression for TPA amplitude (eq 4.2) diverges. This divergence is due to infinite lifetime of excited state in standard response theory. In order to treat this problem the phenomenological lifetime for the excite states which are closed to resonance can be introduced. The finite lifetime for the state $|\mathrm{k}\rangle$ is equivalent to introducing complex excitation energy. Finally, response theory with the phenomenological lifetimes is called the damped response theory. The introduction of the effective excited state lifetimes in terms of the complex excitation energies requires the replacements:

$$
\begin{gathered}
\frac{1}{\omega_{k}-\omega} \rightarrow \frac{1}{\omega_{k}-(\omega+i \gamma)}=D_{k}(\omega)+i A_{k}(\omega) \\
\frac{1}{\omega_{k}+\omega} \rightarrow \frac{1}{\omega_{k}+(\omega+i \gamma)}=D_{k}(-\omega)-i A_{k}(-\omega)
\end{gathered}
$$

where $\omega_{k}$ is an excitation energy, $\omega$ is optical frequency, and $\gamma=\frac{1}{2} \tau^{-1}$ is empirical broadening parameter, where $\tau$ is an effective lifetime common to all excited states, while $D_{k}$ and $A_{k}$ are dispersion and absorption line shape functions. The above explained replacements can be introduced in response equations, thus achieving corrections through empirical parameter $\gamma$ giving rise to physical behavior of molecular properties.

b) Sum over states approach. Alternatively, within the sum over states (SOS) approach for the case where both photons have the same frequency $\omega$, orientationally averaged expressions 
for the two-photon matrix elements for linearly polarized photons with parallel polarization two-photon matrix elements can be formulated as:

$$
\begin{aligned}
\left|T_{f 0}\right|^{2} \sim \sum_{j}^{N} \sum_{k}^{N}\left[\frac{(\langle k|\mu| 0\rangle \cdot\langle f|\mu| k\rangle)(\langle j|\mu| 0\rangle \cdot\langle f|\mu| j\rangle)}{\left[\left(\omega_{k}-\omega\right)\left(\omega_{j}-\omega\right)+\Gamma^{2}\right]}\right. \\
+\frac{(\langle k|\mu| 0\rangle \cdot\langle j|\mu| 0\rangle)(\langle f|\mu| k\rangle \cdot\langle f|\mu| j\rangle)}{\left[\left(\omega_{k}-\omega\right)\left(\omega_{j}-\omega\right)+\Gamma^{2}\right]} \\
\left.+\frac{(\langle k|\mu| 0\rangle \cdot\langle f|\mu| j\rangle)(\langle f|\mu| k\rangle \cdot\langle j|\mu| 0\rangle)]}{\left[\left(\omega_{k}-\omega\right)\left(\omega_{j}-\omega\right)+\Gamma^{2}\right]}\right]
\end{aligned}
$$

The damping factor $\Gamma$ for each one-photon transition serves to prevent the TPA cross sections from obtaining unphysical large values near the one-photon resonances. However, this approach requires explicit calculation of all transition dipole moments among excited states, as well as between them and the ground state which can be realized in the framework of the double residue (DR) approach within quadratic response. Notice that quadratic response functions both single and double residue, the first one is used in analytical formulation presented above, and the second one in the framework of SOS. ${ }^{[7]}$ This allows to adequately correct the TPA cross sections when necessary as well as to include manifold of excited states (usually 20 to 30 ) avoiding "few states" model in which only the dominating terms in the twophoton absorption transition amplitude tensor are accounted for. The influence of the truncation of the SOS on the values of cross sections has to be determined. Usually no changes occur between 20 and 30 states.

\section{Qualitative design of TPA cross sections}

Based on a brief description of theoretical approach it is evident that two factors are contributing to large TPA cross sections entering as nominator and denominator in twophoton absorption amplitude tensor: (i) large dipole transition moments between ground and excited states, as well as among excited states, and (ii) resonance between states involved in 
the OPA and TPA processes. A resonance enhancement factor $\tau$ for TPA defined as $\tau=$ $\left(\frac{E_{i}}{E_{f}}-1 / 2\right)^{-2}$ is usually introduced. Day and coworkers calculated TPA cross sections for each TPA-allowed state versus the resonance enhancement factor $\tau$ for that state demonstrating importance of the resonance enhancement of the TPA cross section for the silver nanoclusters. However, they also showed that a number of states with large resonance enhancement factors have insignificant contributions to the TPA. Thus, in spite of importance of the resonance enhancement factor, also large transition dipole moments between groundstate and excited-state induce large TPA cross sections. ${ }^{[14]}$ Notice also that accurate prediction of OPA is important due to influence of the transition energy values on the suppositions of the spectral range for TPA enhancement. In order to achieve required conditions, in particularly large transition dipole moments, the structural, electronic properties, charge and composition of the nanoclusters has to be accordingly tuned which will be illustrated in Chapters 5 .

From computational point of view, since usually large number of excited states has to be calculated within time dependent density functional theory, the accuracy of the results will depend on limitations of DFT approach such as choice of functionals and inclusion of only single excitations. Therefore, the calculated nonlinear properties such as TPA cross sections should be considered rather of qualitative nature. However, they should provide basis for designing systems with large TPA cross sections stimulating experimental verification.

\section{References.}

[1] P. Sałek, O. Vahtras, T. Helgaker, H. Ågren, Density-functional theory of linear and nonlinear time-dependent molecular properties, Journal of Chemical Physics 2002, 117 (21), pp.9630

[2] P. Sałek, O. Vahtras, J. Guo, Y. Luo, T. Helgarek, H. Ågren, Calculations of two-photon absorption cross sections by means of density-functional theory, Chemical Physics Letter 2003, 374, pp.446 
[3] D. Jonsson, O. Vahtras, B. Jansik, Z. Rinkevicius, P. Sałek, H. Ågren, in Non-Linear Optical Properties of Matter: From Molecules to Condensed Phases (Eds.: M. G. Papadopoulos, A. J. Sadlej, J. Leszczynski), Springer Netherlands, Dordrecht, 2006, pp. 151.

[4] W. Bartkowiak, R. Zaleśny, in Non-Linear Optical Properties of Matter: From Molecules to Condensed Phases (Eds.: M. G. Papadopoulos, A. J. Sadlej, J. Leszczynski), Springer Netherlands, Dordrecht, 2006, pp. 129.

[5] V. P. Gupta, in Principles and Applications of Quantum Chemistry, Academic Press, Boston, 2016, pp. 155.

[6] L. Frediani, Z. Rinkevicius, H. Ågren. Two-photon absorption in solution by means of timedependent density-functional theory and the polarizable continuum model. The Journal of Chemical Physics 2005, 122, 244104.

[7] K. Kristensen, J. Kauczor, T. Kjærgaard, P. Jørgensen. Quasienergy formulation of damped response theory. The Journal of Chemical Physics 2009, 131, 044112.

[8] J Kauczor, Damped response theory, PhD Thesis, The Lundbeck Fundation Center for Theoretical Chemistry, Department of Chemistry, University of Aarhus, December 2010

[9] R. M. Stevens, R. M. Pitzer, W. N. Lipscomb. Perturbed Hartree-Fock Calculations. I. Magnetic Susceptibility and Shielding in the LiH Molecule. The Journal of Chemical Physics 1963, 38, 550.

[10] P. Norman, D. M. Bishop, H. J. A. Jensen, J. Oddershede, Nonlinear response theory with relaxation: The first-order hyperpolarizability, The Journal of Chemical Physics, 2005, 123, 194103

[11] P. Norman, A perspective on nonresonant and resonant electronic response theory for timedependent molecular properties, Physical Chemistry Chemical Physics, 2011, 13, pp.20519

[12] P. Norman, K. Ruud, in Non-Linear Optical Properties of Matter: From Molecules to Condensed Phases (Eds.: M. G. Papadopoulos, A. J. Sadlej, J. Leszczynski), Springer Netherlands, Dordrecht, 2006, pp. 1.

[13] K. Kristensen, J. Kauczor, A. J. Thorvaldsen, P. Jørgensen, T. Kjærgaard, A. Rizzo. Damped response theory description of two-photon absorption. The Journal of Chemical Physics 2011, 134, 214104.

[14] P. N. Day, R. Pachter, K. A. Nguyen, T. P. Bigioni. Linear and Nonlinear Optical Response in Silver Nanoclusters: Insight from a Computational Investigation. The Journal of Physical Chemistry A 2016, 120, 507. 


\section{Chapter 5}

\section{Design Strategy and Structure-Property Relation for Enhanced Two-Photon Absorption in Ligated Metal Nanoclusters.}

\section{Introduction.}

A complete understanding of the two-photon absorption (TPA) process is a key issue for the rational design of optimal two-photon chromophores. For this purpose structure-property relationship of molecular TPA is of great importance. In this chapter, taking example of pushpull molecules, we will describe first how theoretical results can be used to obtain an extensive comprehension of the physics underlying the two-photon process and its amplitude. This will serve to propose an exploratory root for novel chemical engineering leading to further enhancement of TPA in liganded silver and gold quantum clusters. Using small ligated silver nanoclusters as test systems, we illustrate how theoretical approaches together with experimental findings can contribute to the understanding of structure-property relationships that might ultimately guide nanocluster synthesis. 
Molecular models for large TPA cross section. Push-pull molecules.

Push-pull dipolar molecules are characterized by a low-lying, high-intensity absorption band, related to the intramolecular charge transfer (ICT) between the electron donor (D) and acceptor (A) groups (see Figure 5.1). ${ }^{[1,2]}$

a)
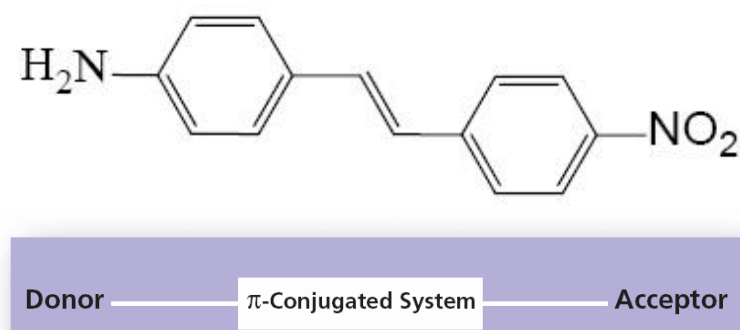

b)

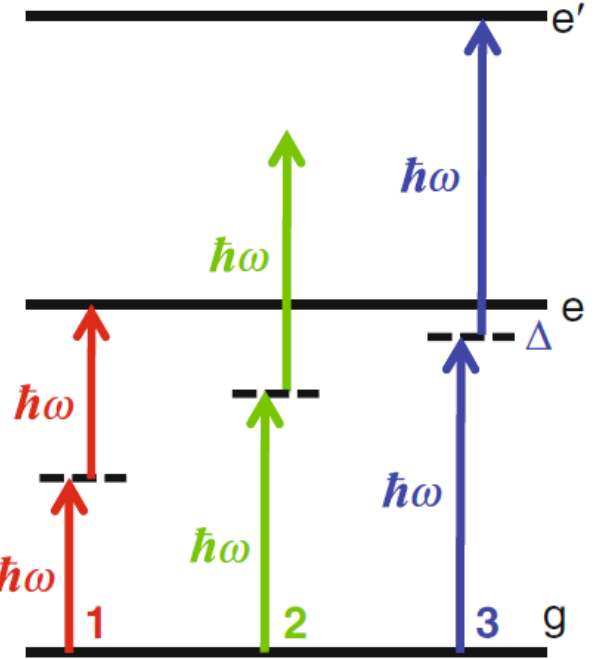

Figure 5.1: a) Schematic illustration of a nonlinear optical (NLO), push-pull chromophore. b) Schematic of frequency degenerate TPA (1) into the first allowed singlet state, (2) above the first allowed singlet state, and (3) a double resonant condition, with a small intermediate state resonance energy difference, D, and a transition into an allowed final TPA state.

The TPA cross section of such molecules is considered to be governed basically by two factors: transition dipole moments and transition energies of the molecule. As seen below, the theoretical expression of TPA cross section $\left(\delta_{\mathrm{TPA}}\right)$ based on the perturbation expansion is comprised of the numerator including transition dipole moments and the denominator including the transition energies and the incident photon energy. The energy term determines the wavelength dispersion of $\delta_{\text {TPA }}$ whereas the dipole moment term determines the overall magnitude of $\delta_{\text {TPA }}$. Thus, the structure-property relationship for the molecules with large $\delta_{\text {TPA }}$ can be reduced by optimizing the interplay between the transition dipole moments and frequencies involved in the TPA process.

As described in chapter 4 , the TPA cross section at the laser frequency of $\omega$ is given as 


$$
\delta_{T P A}=\frac{(2 \pi e)^{4} \omega^{2}}{c^{2}} g(2 \omega)\left|T^{2 \omega, f}\right|^{2}
$$

where $g(2 \omega)$ is normalized line-shaped function and $T^{2 \omega, f}$ is the two-photon absorption amplitude tensor. Experimental measurement produces average TPA cross section which can be evaluated by averaging the two-photon transition amplitude tensor $\left|T^{2 \omega, f}\right|^{2}$ over all orientations. Cartesian components $T_{a b}^{2 \omega, f}$ between the ground state $|0\rangle$ and the excited state $|f\rangle$ are defined as:

$$
T_{a b}^{2 \omega, f}=\sum_{k>0}\left[\frac{\left\langle 0\left|\hat{\mu}_{a}\right| k\right\rangle\left\langle k\left|\hat{\mu}_{b}\right| f\right\rangle}{\omega_{k}-\omega_{f} / 2}+\frac{\left\langle 0\left|\hat{\mu}_{b}\right| k\right\rangle\left\langle k\left|\hat{\mu}_{a}\right| f\right\rangle}{\omega_{k}-\omega_{f} / 2}\right](a, b=x, y, z)
$$

where frequency of incoming radiation is equal to half of this excitation energy from the ground state $|0\rangle$ to the excited state $|f\rangle$, with $\omega_{k}=\omega_{f} / 2$. The quantities $\mu_{a}$ and $\mu_{b}$ are the Cartesian components of the dipole moments operator $\hat{\mu}$ and $\omega_{k}$ and $\omega_{f}$ are the frequencies of the excitation from $|0\rangle$ to $|k\rangle$ and $|f\rangle$, respectively.

Enhancement of $\delta_{T P A}$ can be obtained by playing with the following factors :

1. Increasing the transition dipole moments. In molecular design, this can be realized by introducing electron donor/acceptor groups. In non-centrosymmetric molecules, increasing the difference of the ground and excited state permanent dipole moments can also increase $\delta_{T P A}(\omega)$.

2. Maximizing resonance terms. Decreasing the detuning energy between intermediate and ground states can significantly enhance $\delta_{T P A}(\omega)$. If the intermediate state is located halfway between ground state and final state, a "double resonance" condition can be achieved, which can lead to a dramatic enhancement of $\delta_{T P A}(\omega)$. 
Such factors have been figured out in details for design strategies and structure-property relations of cyanine and cyanine-like molecular structures with the goal of enhancing TPA in the near-IR for multiphoton fluorescence sensing applications. ${ }^{[3]}$

\section{Ligand-Core NLO-phores: Concepts for Design of Enhanced two-photon absorption of small ligated silver nanoclusters.}

Size specific nanoclusters of silver or gold can be viewed as a "multi-shell system" (see fig. 2.7), where shells may communicate in two different ways : charge transfer from ligand to metal core (analogy with ligand-to-metal charge transfer (LMCT) or ligand-to metal- metal charge transfer (LMMCT) observed in metal complexes) and through direct bonding or direct donation of delocalized electrons of electron-rich groups of the ligands. ${ }^{[4]}$ Such "communications" between ligands and metal core may increase the transition dipole moments leading to enhanced $\delta_{\mathrm{TPA}}$.

As an illustration, we choose as model system : the $\mathrm{Ag}_{15} \mathrm{~L}_{11}$ cluster (with $\mathrm{L}=\mathrm{SH}$ ) containing an $\mathrm{Ag}_{8}$ core which is protected by four ligands belonging to three different types. Three types of ligands are present: L1 (HS-Ag-SH), L2 (HS-Ag-SH-Ag-SH) and L3 type (HS-Ag-SH$\mathrm{Ag}-\mathrm{SH}-\mathrm{Ag}-\mathrm{SH})$. The optimized structure of $\mathrm{Ag}_{15}(\mathrm{SH})_{11}$ as well as the core with two L1, and one L2 and L3 are shown in Fig. 5.2a. The one photon absorption (OPA) spectrum of $\mathrm{Ag}_{15}(\mathrm{SH})_{11}$ along with the analysis of the four first transitions is given in Fig. 5.2a. These transitions arise from an excitation from the P-cluster-core orbital to the D-cluster-coreorbitals labelled by "core-core". $\mathrm{S}_{2}, \mathrm{~S}_{3}, \mathrm{~S}_{4}$ transitions are located between $390 \mathrm{~nm}$ and 450 $\mathrm{nm} \cdot{ }^{[5]}$ 


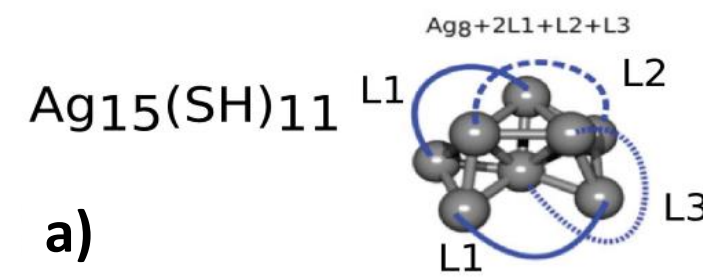

L3

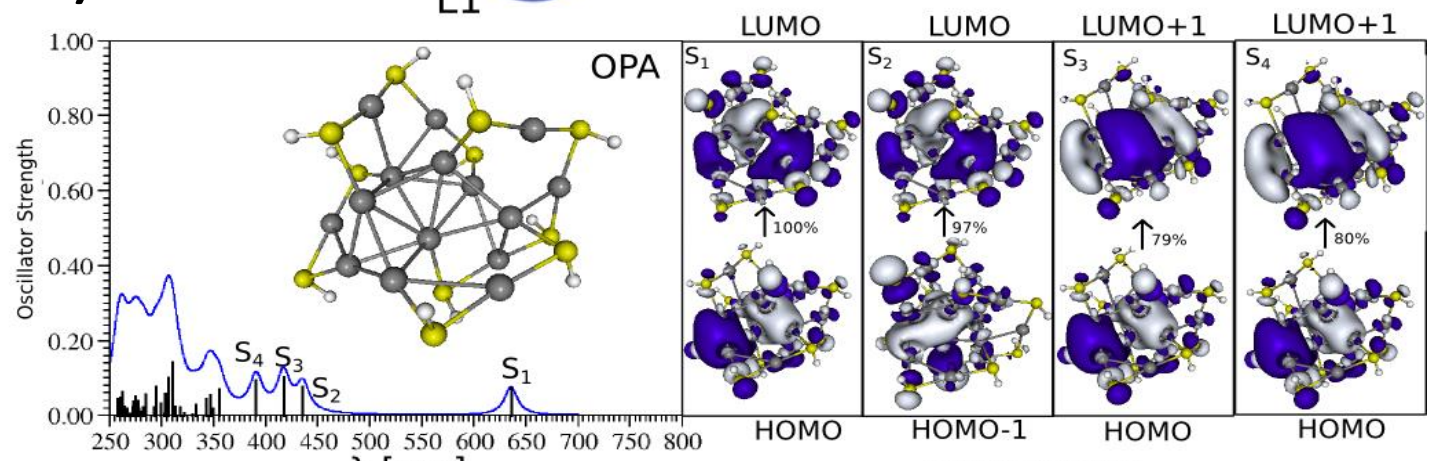

b)

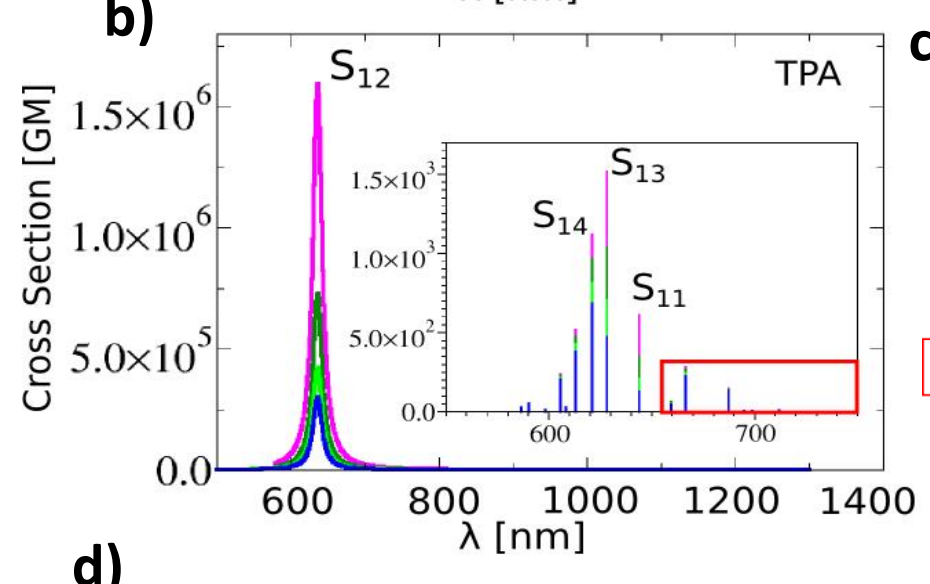

CORE-CORE

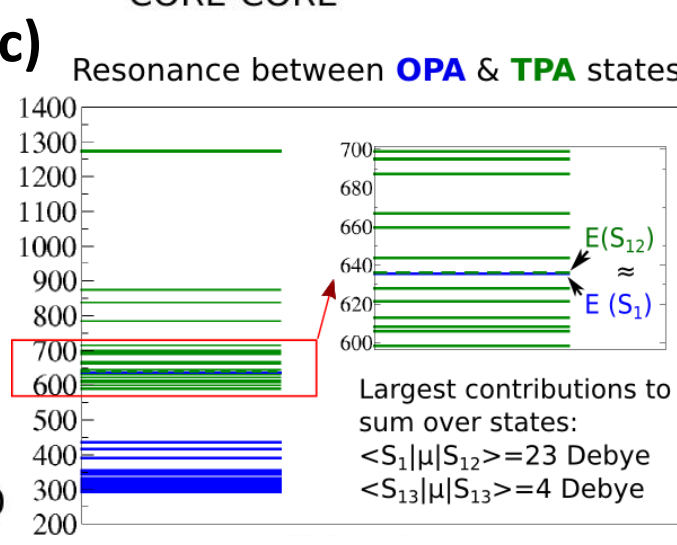

d)
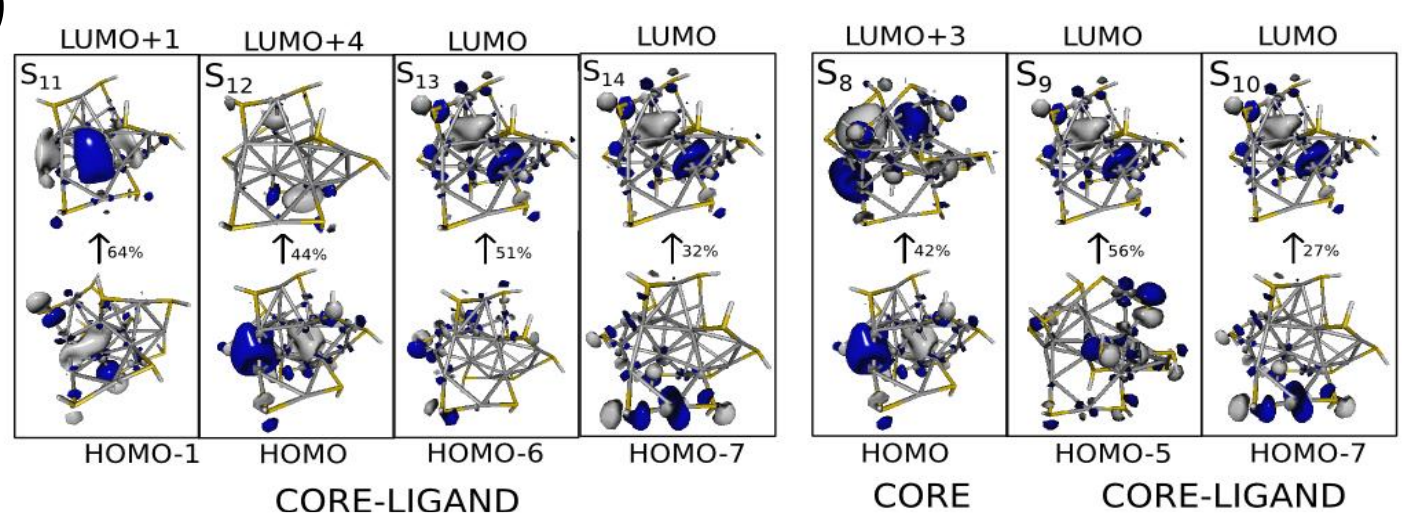

Figure 5.2: (a) The lowest energy structure of $A_{15} L_{11}$ (with an $A_{8}$ core and staple ligands $L_{n}$ where $n$ is the number of $A g$ atoms involved in the staple motif). One-photon absorption spectrum for the lowest energy of $\mathrm{Ag}_{15}(\mathrm{SH})_{11}$ obtained with TDDFT method using CAM-B3LYP functional and def2-TZVP basis set. Analysis of transitions of absorption for $\operatorname{Ag}_{15}\left(\mathrm{SH}_{11}\right.$ in terms of leading excitations between Kohn-Sham orbitals. (b) Two-photon absorption spectrum obtained with quadratic response QR-DFT method using sum over states (SOS) approach allowing to include a damping factor for each one photon transition ( $\Gamma=0.02$, violet; $\Gamma=0.03$, green; $\Gamma=0.04$, red and $\Gamma=0.05$, blue). c) One-photon absorption spectrum for the lowest energy of $\mathrm{Ag}_{15}(\mathrm{SH})_{11}$ obtained with TDDFT method using CAM-B3LYP functional and def2-TZVP basis set. d) Analysis of transitions of two-photon absorption for $\operatorname{Ag}_{15}(\mathrm{SH})_{11}$ in terms of leading excitations between Kohn-Sham orbitals. 
The two-photon absorption (TPA) spectrum for the optimized structure of $\mathrm{Ag}_{15} \mathrm{~L}_{11}$ obtained with the quadratic response QR-DFT method and the sum over states (SOS) approach allowing to include a dumping factor for each one photon transition is presented in Fig. 5.2b. A large resonance enhancement occurs when the excitation energy of an OPA state is close to half that of a TPA state ("double resonance" condition). This is nicely illustrated in Fig. 5.2c, with $S_{1}(630 \mathrm{~nm})$ and $S_{12}(315 \mathrm{~nm})$. In fact, the first excited state at $630 \mathrm{~nm}$ is in resonance with several states near $315 \mathrm{~nm}$, resulting in an unphysically large calculated TPA of over $10^{6} \mathrm{GM}$ (see Fig. 5.2b) which needs to be corrected by damping factors. Notice that the absolute value of the cross section for TPA is found to be strongly dependent on the value of the damping factor, e.g. for damping factor of $\Gamma=0.05$, the TPA cross section $\sigma$ is 300000 GM. Indeed, the damping factor for two-photon transitions prevents the TPA cross section from blowing up near a one-photon resonance. Nevertheless, notice that the largest contributions of transition dipole moments to the sum-over states are $\left\langle\mathrm{S}_{1}|\mu| \mathrm{S}_{12}\right\rangle=23 \mathrm{D}$ and $\left\langle\mathrm{S}_{13}|\mu| \mathrm{S}_{13}\right\rangle=4 \mathrm{D}$ involve $\mathrm{S}_{12}$ and $\mathrm{S}_{13}$ excited states. Indeed, the leading excitations corresponding to large TPA cross sections involve the ligands' orbitals and not only the core's ones (see fig. 5.2d). In conclusion, both double resonance and transition dipole moments contribute to the large value of TPA cross section.

We carried out a theoretical investigation of the nonlinear optical properties for the lowest energy structures of the $A_{11} L_{7}, A_{15} L_{11}$ and $A g_{31} L_{19}$ nanoclusters, where $L$ stands for the $\mathrm{SCH}_{3}$ group. ${ }^{[6]}$ They contain respectively 4,8 and 12 delocalized electrons within the core. Several factors influencing the TPA cross-sections have been figure out: (i) the excitation between ligands and the metal core characterize the nonlinear transitions, (ii) the "double resonance" between states involved in the OPA and TPA processes is required to obtain giant TPA cross-sections, which can be corrected to realistic values (iii) large dipole transition 
moments are related to a non-uniform electron distribution within the metal core. The role of the structural properties, i.e. of the geometry of the metal core in determining this electron distribution, is therefore crucial.
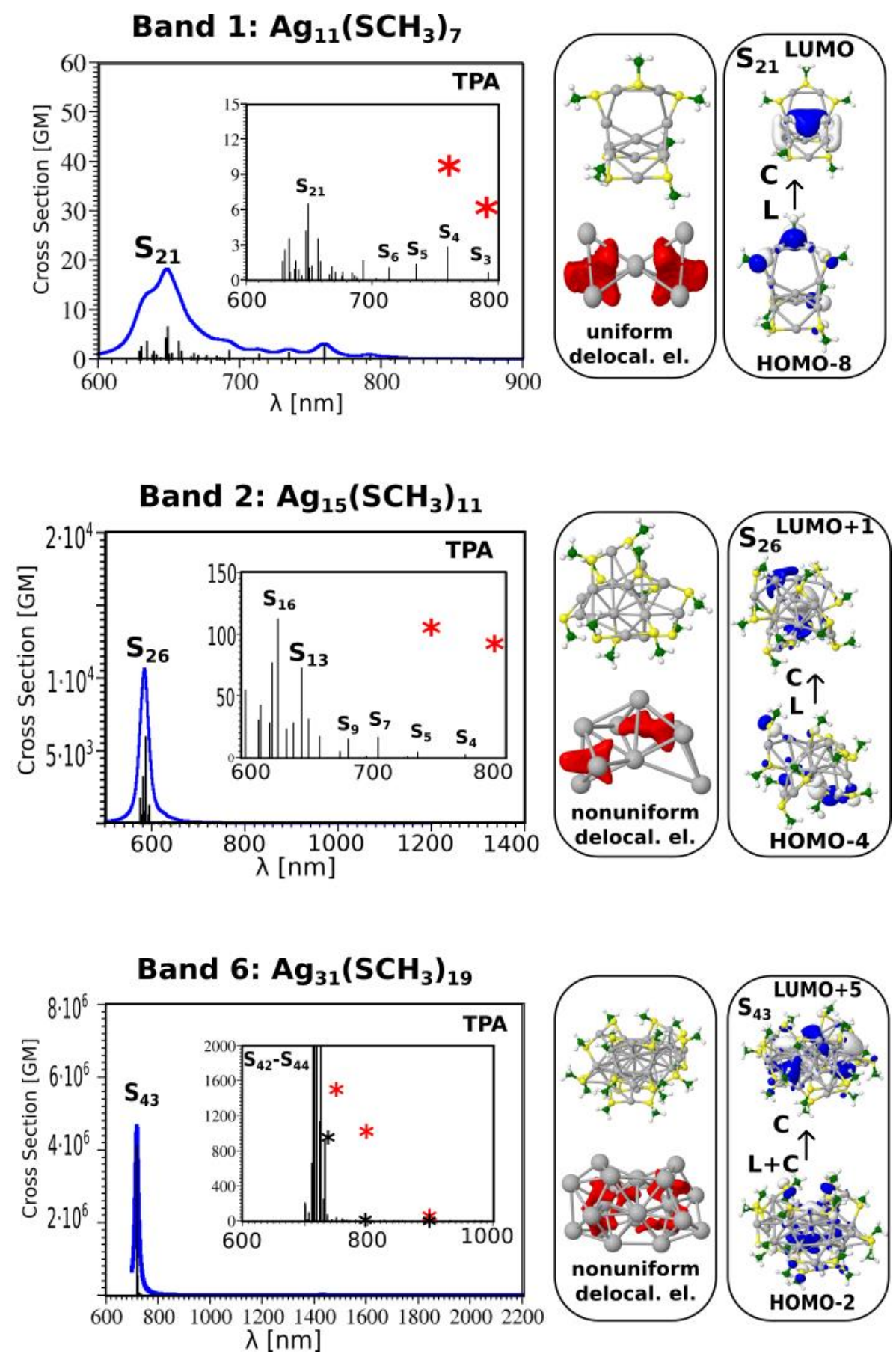

Figure 5.3: Comparison of TDDFT TPA spectra obtained by quadratic response approach of $\mathrm{Ag}_{11}\left(\mathrm{SCH}_{3}\right)_{7}, \mathrm{Ag}_{15}\left(\mathrm{SCH}_{3}\right)_{11}$ and $\mathrm{Ag}_{31}\left(\mathrm{SCH}_{3}\right)_{19}$ nanoclusters using for silver atoms the 19-e relativistic effective core potential (19-e RECP) taking into account scalar relativistic effects and for all atoms triple zeta plus polarization AO basis set (TZVP) together with CAMB3LYP functional (for details cf. ref. ${ }^{[6]}$ ). The lowest energy structures contain 4, 8 and 12 delocalized electrons in the metal core. Red asterisks label experimental values, while the black ones label theoretical values. (adapted from ref. ${ }^{[6]}$ ). 
Damping factor of 0.02 is used for $\mathrm{Ag}_{15}\left(\mathrm{SCH}_{3}\right)_{11}$ yielding lower TPA cross-section values within 2 orders of magnitude. For $\mathrm{Ag}_{31}\left(\mathrm{SCH}_{3}\right)_{19}$, a three state model is used accounting for only leading transition dipole moment contributions to the TPA cross section avoiding the calculation for transition dipole moments of all excited states. This allows correcting the values of the TPA cross-sections for $\mathrm{S}_{43}$ within 4 orders of magnitude, instead of using the damping factor. Structures of the clusters together with the electron localization function (ELF) representing delocalized electrons within the core plotted for the isovalue $\mathbf{0 . 2 0}$. Leading excitations responsible for the large TPA cross-sections illustrating the participation of the ligands and the core are also shown.

The impact of these factors is illustrated in Fig. 5.3 and 5.4. In the case of the $\operatorname{Ag}_{11} \mathrm{~L}_{7}$ nanoclusters, the "double resonance" between the OPA process with an excited $\mathrm{S}_{1}$ state localized at a wavelength of about $450 \mathrm{~nm}$ can be achieved only by calculations of nonrealistic high number of states. In contrast, for the $\mathrm{Ag}_{15} \mathrm{~L}_{11}$ and $\mathrm{Ag}_{31} \mathrm{~L}_{19}$ nanoclusters, there is a "double resonance" between the low lying states accessible through OPA and TPA in the spectral range around $590 \mathrm{~nm}$ (in the case of $\mathrm{Ag}_{15} \mathrm{~L}_{11}$ ) and $730 \mathrm{~nm}$ (in the case of $\mathrm{Ag}_{31} \mathrm{~L}_{19}$ ).

Also, a non-uniformity of the delocalized electron distributions within the core is a prerequisite for large transition dipole moments of LMMCT excitations involving ligands and the metal core. The influence of changing the charge distribution from uniform to nonuniform on the values of cross-sections is illustrated in the case of $\operatorname{Ag}_{11} \mathrm{~L}_{7}$ by introducing a positive external charge on the central $\mathrm{Ag}$ atom of the metal core. The redistribution of electron density causes the increase of transition dipole moments through LMMCT excitations and induces an increase in TPA, as shown in Fig. 5.4. 


\section{$\operatorname{Ag}_{11}\left(\mathrm{SCH}_{3}\right)_{7}$}
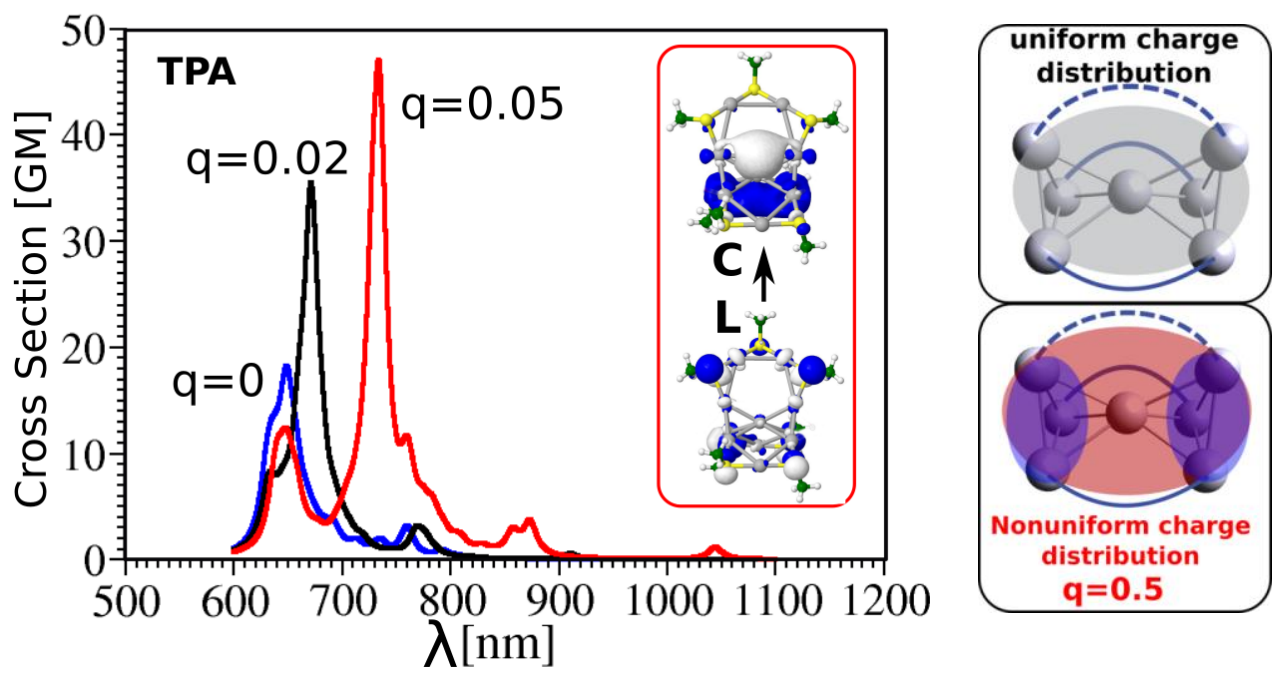

Figure 5.4: Comparison of TDDFT TPA spectra of $\mathrm{Ag}_{11}\left(\mathrm{SCH}_{3}\right)_{7}$ without and with an external charge on the central $\mathrm{Ag}$ atom illustrating an increase of transition dipole moments (shown on the left side) as well as transition from uniform delocalized electrons $(q=0)$ to non-uniform delocalized electrons $(q=0.05)$ (shown on the right side). Blue - positive, red - negative delocalization of electronic charge. Leading excitation from the ligand to the core for the considered excited state in inset. Values of maximal transition dipole moments (Debye) and cross sections (GM) for three values of external charge: $q=0$ are 4.2 Debye (6 GM), $q=0.02$ are 4.6 Debye (26 GM) and $q=0.05$ are 16.3 Debye (36 GM). (adapted from ref. ${ }^{[6]) .}$

\section{Design strategy and structure-property relations.}

For the magnitude of the TPA cross-sections, interplay between a resonance effect and large transition dipole moments is essential. Large transition dipole moments are due to ligand-tocore or inversely core-to-ligand excitations, and are reinforced by a non-uniform electronic distribution in the metal core. The latter features are consequences not only of the size but of the structural properties of the metal core. Concerning the importance of non-uniform electronic distribution in the metal core on NLO properties, $\mathrm{Au}_{25}{ }^{\mathrm{q}}$ clusters have a tunable ground state charge $(\mathrm{q}=-1,0,+1)$, their crystal structure has been solved. ${ }^{[7]}$ These characteristics allow differently charged $\mathrm{Au}_{25}{ }^{\mathrm{q}}$ clusters to function as well-defined models for probing the impact on NLO properties of charged active sites.

Also, the optical band gap decreases as the size of the nanoclusters increases, ${ }^{[8]}$ shifting the spectra towards the NIR spectral region, a feature useful for bio-imaging applications. ${ }^{[9]}$ In 
addition, the structural properties of the metal core in the size regime with molecular-like behaviour play an important role. It has indeed been recently reported by Pradeep and collaborators $^{[10]}$ that metallicity in silver nanoclusters emerges for core sizes of about 150 atoms. For such sizes governed by the plasmonic excitations, ligands have substantional less important role. They modify the NLO properties that stem from the surface in the quadratic nonlinearity regime. In this case, the Ag-S bond is usually detrimental. ${ }^{[11]}$ In the cubic regime, the NLO properties depend on the volume of the core and are therefore not as much influenced by the ligands. Altogether, understanding of leading factors governing non-linear properties of ligated small silver nanoclusters allows proposing novel ligand-core NLOphores with potential for different applications.

\section{References}

[1] F. Meyers, J. L. Bredas. Electronic structure and nonlinear optical properties of push-pull conjugated molecules. Int. J. Quantum Chem. 1992, 42, 1595.

[2] F. Terenziani, C. Katan, E. Badaeva, S. Tretiak, M. Blanchard-Desce. Enhanced Two-Photon Absorption of Organic Chromophores: Theoretical and Experimental Assessments. Advanced Materials 2008, 20, 4641.

[3] O. V. Przhonska, S. Webster, L. A. Padilha, H. Hu, A. D. Kachkovski, D. J. Hagan, E. W. Van Stryland, in Advanced Fluorescence Reporters in Chemistry and Biology I: Fundamentals and Molecular Design (Ed.: A. P. Demchenko), Springer Berlin Heidelberg, Berlin, Heidelberg, 2010, pp. 105.

[4] Z. Wu, R. Jin. On the Ligand's Role in the Fluorescence of Gold Nanoclusters. Nano Letters 2010, 10, 2568.

[5] Z. Sanader, M. Krstic, I. Russier-Antoine, F. Bertorelle, P. Dugourd, P.-F. Brevet, R. Antoine, V. Bonacic-Koutecky. Two-photon absorption of ligand-protected Ag15 nanoclusters. Towards a new class of nonlinear optics nanomaterials. Physical Chemistry Chemical Physics 2016, 18, 12404.

[6] I. Russier-Antoine, F. Bertorelle, N. Calin, Z. Sanader, M. Krstic, C. Comby-Zerbino, P. Dugourd, P.-F. Brevet, V. Bonacic-Koutecky, R. Antoine. Ligand-core NLO-phores: a combined experimental and theoretical approach to the two-photon absorption and two-photon excited emission properties of small-ligated silver nanoclusters. Nanoscale 2017, 9, 1221. 
[7] D. R. Kauffman, D. Alfonso, C. Matranga, P. Ohodnicki, X. Deng, R. C. Siva, C. Zeng, R. Jin. Probing active site chemistry with differently charged Au25q nanoclusters $(q=-1,0,+1)$. Chemical Science 2014, 5, 3151.

[8] R. Jin. Atomically precise metal nanoclusters: stable sizes and optical properties. Nanoscale 2015, 7, 1549.

[9] L.-Y. Chen, C.-W. Wang, Z. Yuan, H.-T. Chang. Fluorescent Gold Nanoclusters: Recent Advances in Sensing and Imaging. Analytical Chemistry 2015, 87, 216.

[10] I. Chakraborty, J. Erusappan, A. Govindarajan, K. S. Sugi, T. Udayabhaskararao, A. Ghosh, T. Pradeep. Emergence of metallicity in silver clusters in the 150 atom regime: a study of differently sized silver clusters. Nanoscale 2014, 6, 8024.

[11] J. Nappa, G. Revillod, I. Russier-Antoine, E. Benichou, C. Jonin, P. F. Brevet. Electric dipole origin of the second harmonic generation of small metallic particles. Physical Review B 2005, $71,165407$. 


\section{Chapter 6}

\section{Measurement Techniques of Optical Nonlinearities.}

\section{Two-photon absorption/fluorescence and Hyper-Rayleigh scattering}

\section{Introduction.}

There is considerable interest in finding nanomaterials exhibiting large optical nonlinearities. Thus, there is a need to develop methods to determine nonlinear coefficients discussed throughout this book. The Z-scan technique is a method able to measure both nonlinear absorption (NLA) and nonlinear refraction (NLR) in solids, liquids and liquid solutions. In this chapter we first present a brief review of this technique. Also the "P-scan" technique developed in our experimental lab will be described along with the photon detection set-up which permits to measure two-photon excited fluorescence cross sections. We will also describe the physical principles that allow the realization of Hyper-Rayleigh scattering. We will compare the incoherent Hyper-Rayleigh scattering (HRS) technique with a coherent experimental technique: Electric-Field- Induced Second-Harmonic Generation (EFISHG). Hyper-Rayleigh technique can be employed to characterize the first hyperpolarizability of nanoclusters in solution. 


\section{Hyper-Ryleigh scattering}

\section{a) Incoherent and coherent nonlinear characterization}

Coherent techniques take advantage of macroscopic ordering of the molecules to generate a signal where the contributions of each chromophore add coherently. The relatively small nonlinearity of the molecules can be overcome by measuring the overall coherent signal and relating the macroscopic measurements to the molecular properties.

EFISHG is the coherent technique that allows the response of chromophores in solution to be measured. In the EFISHG technique the centrosymmetry of the solution is broken by applying a strong DC electric field. This field induces an average orientation of the molecules that results in coherent second-harmonic signal. As a result, this technique is only limited to the measurement of $\beta$ of molecules in solution with dipolar symmetry. ${ }^{[1,2]}$

Unlike EFISHG, Hyper-Rayleigh scattering can be used to directly measure properties of all molecules, irrespective of symmetry or charge. As a result, the HRS technique has been used for the measurement of $\beta$ 's of nanomaterials. Unlike EFISHG, Hyper-Rayleigh scattering ${ }^{[3,4]}$ is a non-coherent technique and the efficiencies are rather low. A high intensity incoming laser beam is thus desirable in order to increase the intensity of the small signal and to obtain a better ratio between signal and noise.

\section{b) Hyper-Rayleigh Scattering principles}

Hyper-Rayleigh Scattering is the non-linear equivalent of Rayleigh scattering where the nonlinear terms in the molecular response function are introduced (see chapter 3). The expression for the ith component of the nonlinear scattered light intensity, $I_{i}^{(2 \omega)}$ as a function of the components of the incident light intensity, $I_{i j}^{(\omega)}$ is :

$I_{i}^{(2 \omega)}=C \omega^{4} \sum_{j} \beta_{i j j}(-2 \omega ; \omega, \omega)\left(I_{i j}^{(\omega)}\right)^{2}$ 
The constants of proportionality $C$ is related to the distance and positions of the observer and the scattering particles. Aside from the frequency dependence of the nonlinear molecular susceptibility, $\beta_{i j j}(-2 \omega ; \omega, \omega)$, the intensity is proportional to the fourth power of the frequency.

Only non-centrosymmetrical molecules show even-order scattering, therefore it is possible to dissolve non-centrosymmetric molecules in centrosymmetrical solvents and measure only the intensity of the second-order scattered light generated by the dissolved molecules. As in the case of linear Rayleigh scattering, fluctuations play a fundamental role in the detection of second-order nonlinear light scattering. The rotational fluctuations destroy the average isotropy in the solution locally in time and space. Spatial and temporal orientational fluctuations result in detectable Hyper-Rayleigh Scattering signal.

A Hyper-Rayleigh scattering experiment is performed by measuring the intensity of the incoherently scattered frequency-doubled light generated by an intense laser beam from an isotropic solution. The scattered intensity of a single molecule at the harmonic wavelength can be calculated by performing an orientational average over $\beta_{H R S}:^{[5]}$

$I_{2 \omega}=\frac{32 \pi^{2}}{\epsilon_{0}^{3} \lambda^{4} r^{2}}\left\langle\beta_{H R S}^{2}\right\rangle I_{\omega}^{2}$

where the brackets indicate the orientational averaging, $\lambda$ is the fundamental wavelength and $r$ is the distance to the scattering molecule. Assuming that the molecules in the scattering volume are independent, the total intensity is proportional to the sum of the intensities scattered by the individual molecules:

$I_{2 \omega}=\frac{32 \pi^{2}}{c \epsilon_{0}^{3} \lambda^{4} r^{2}} N\left(f_{\omega}\right)^{4}\left(f_{2 \omega}\right)^{2}\left\langle\beta_{H R S}^{2}\right\rangle I_{\omega}^{2}$

where $c$ the speed of light in vacuum, $\epsilon_{0}$ is the permittivity of free space $N$ is the concentration of chromophores, and $f_{\omega}, f_{2 \omega}$ are the local field factors that take into account the dielectric effects of the environment around the molecules. 

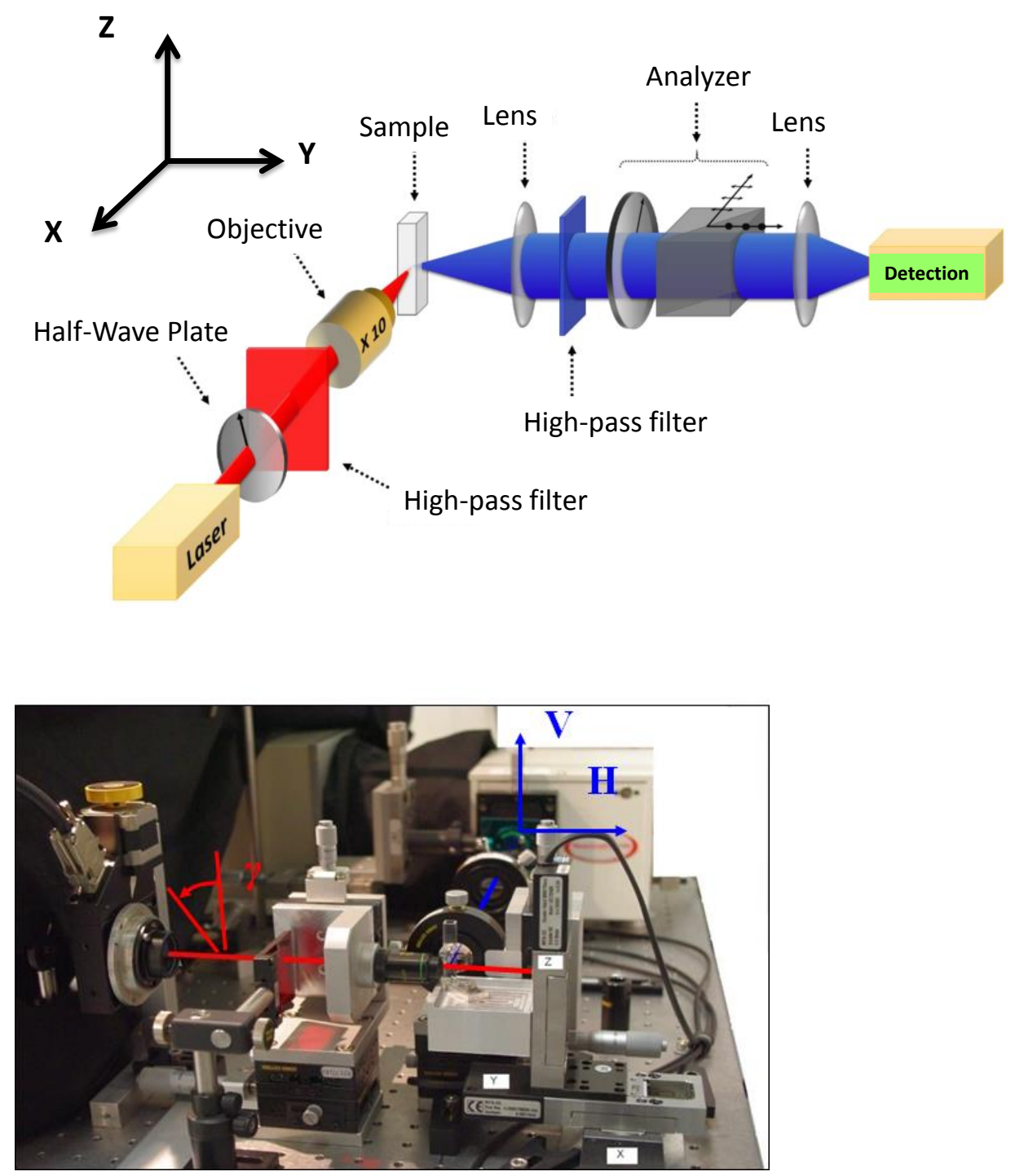

Figure 6.1: Schematic view and photo of the classical $90^{\circ}$ angle Hyper-Rayleigh scattering geometry. An intense laser beam $\left(I_{\omega}\right)$ is brought to focus through a cell containing the isotropic solution and the frequency-doubled light, $\left(I_{2 \omega}\right)$, is collected and detected at $90^{\circ}$ with respect to the direction of propagation of the fundamental beam.

The scattering geometry and the polarization state of both the fundamental and the harmonic light beams determine the relationship between $\beta_{H R S}$ and the molecular tensor components $\beta_{i j k}$. In classical Hyper-Rayleigh scattering experiments, the $90^{\circ}$ angle geometry is mainly used (see figure 6.1). The set-up is built in such a way that the fundamental light beam is propagating in the $X$-direction and polarized in the $Z$-direction, and the scattered light is collected in the $Y$-direction (see Fig. 6.1). We distinguish between the laboratory coordinate 
system of reference $(X, Y, Z)$, and the molecular coordinate system of reference $(x, y, z)$. For such measured geometry the relation between the orientationally averaged tensor components and the molecular tensor components can be expressed as: ${ }^{[4]}$

$\left\langle\beta_{Z Z Z}^{2}\right\rangle=\frac{1}{7} \sum_{i} \beta_{i i i}^{2}+\frac{6}{35} \sum_{i \neq j} \beta_{i i i} \beta_{i j j}+\frac{9}{35} \sum_{i \neq j} \beta_{i i j}^{2}+\frac{6}{35} \sum_{i j k, c y c l i c} \beta_{i i j} \beta_{j k k}+\frac{12}{35} \beta_{i j k}^{2}$

$\left\langle\beta_{X Z Z}^{2}\right\rangle=\frac{1}{35} \sum_{i} \beta_{i i i}^{2}-\frac{2}{105} \sum_{i \neq j} \beta_{i i i} \beta_{i j j}+\frac{11}{105} \sum_{i \neq j} \beta_{i i j}^{2}-\frac{2}{105} \sum_{i j k, c y c l i c} \beta_{i i j} \beta_{j k k}+\frac{8}{35} \beta_{i j k}^{2}$

The first subscript ( $\mathrm{X}$ or $\mathrm{Z}$ ) refers to the polarization state of the frequency doubled light (in the laboratory coordinate system). Since both polarizations are detected with equal sensitivity, and the fundamental light beam is polarized vertically, the orientational average over $\beta$ is the sum of both contributions:

$\left\langle\beta_{H R S}^{2}\right\rangle=\left\langle\beta_{Z Z Z}^{2}\right\rangle+\left\langle\beta_{X Z Z}^{2}\right\rangle$

The orientational averaged hyperpolarizability squared $\left\langle\beta_{H R S}^{2}\right\rangle$ is related to the molecular hyperpolarizability tensor components according to Eqs. 6.5 and 6.6.

\section{c) Experimental Hyper-Rayleigh scattering set-up}

A schematic view of the set-up is shown in Figure 6.1. In our experimental group, the light source for the HRS experiments is a mode-locked femtosecond Ti:sapphire laser produced pulses at the fundamental wavelength of $800 \mathrm{~nm}$ with a duration of about 140 femtoseconds at a repetition rate of $76 \mathrm{MHz}$. After passing through a low-pass filter, in order to remove any unwanted harmonic light generated prior to the cell, the fundamental beam of about $300 \mathrm{~mW}$ is focused by a microscope objective into a $1 \mathrm{~cm} \times 1 \mathrm{~cm}$ spectrophotometric cell containing 
the aqueous solution. The HRS light is collected at an angle of $90^{\circ}$ from the incident direction by a $2.5 \mathrm{~cm}$ focal length lens. The second harmonic light is separated from its linear counterpart by a high-pass filter and a monochromator positioned at the second harmonic wavelength. The HRS light is then detected with a photomultiplier tube and the pulses produced counted with a photon counter. The fundamental beam is chopped at about $115 \mathrm{~Hz}$ to enable a gated photon counting mode allowing automatic subtraction of the noise level.

In these experiments, where the HRS intensity is collected, the monochromaticity of the second harmonic light generated was always assessed to prevent any spurious contributions from luminescence. Finally, the HRS intensity for different concentration was normalized against that of the bare solvent. At each concentration, a $20 \mathrm{~nm}$ narrow band spectrum around the HRS line was recorded, see Figure 6.2. This experimental procedure is deemed necessary to ensure that the process indeed corresponds to the conversion of two photons at the fundamental frequency into one photon at the harmonic frequency. It is observed in particular that the HRS line is located on top of a strong broadband photoluminescence background. The subtraction of the photoluminescence background was then performed by fitting the narrow band background spectra with a Gaussian function for the HRS line superposed on a linear function of the wavelength accounting for the broadband luminescence background. This subtraction procedure is allowed since the two processes, photoluminescence and HRS, are incoherent. The HRS intensity is the given by :

$$
\frac{I_{H R S}}{I_{H R S}^{w}}=\frac{\left\langle N_{w} \beta_{w}^{2}+N \beta^{2}\right\rangle}{\left\langle N_{w} \beta_{w}^{2}\right\rangle}=1+b^{\prime} N\left\langle\beta^{2}\right\rangle
$$

where the subscripts $w$ stands for water which is the reference solvent used in this experiment. The losses due to absorbance at the fundamental and the harmonic frequency are determined 
from the separate UV-visible absorption measurements and all data are corrected prior to the analysis. The HRS intensity recorded for different concentrations of $\mathrm{Au}_{15} \mathrm{SG}_{13}$ in aqueous solution is reported in Figure 6.2.

As seen in Eq. 6.7, it is necessary to know the hyperpolarizability of the bare solvent to determine the hyperpolarizability of the nanoclusters. We use the value of $0.08710^{-30}$ esu for the first hyperpolarizability of the neat water solution. A discussion about this value may be found in a previous work. ${ }^{[6]}$

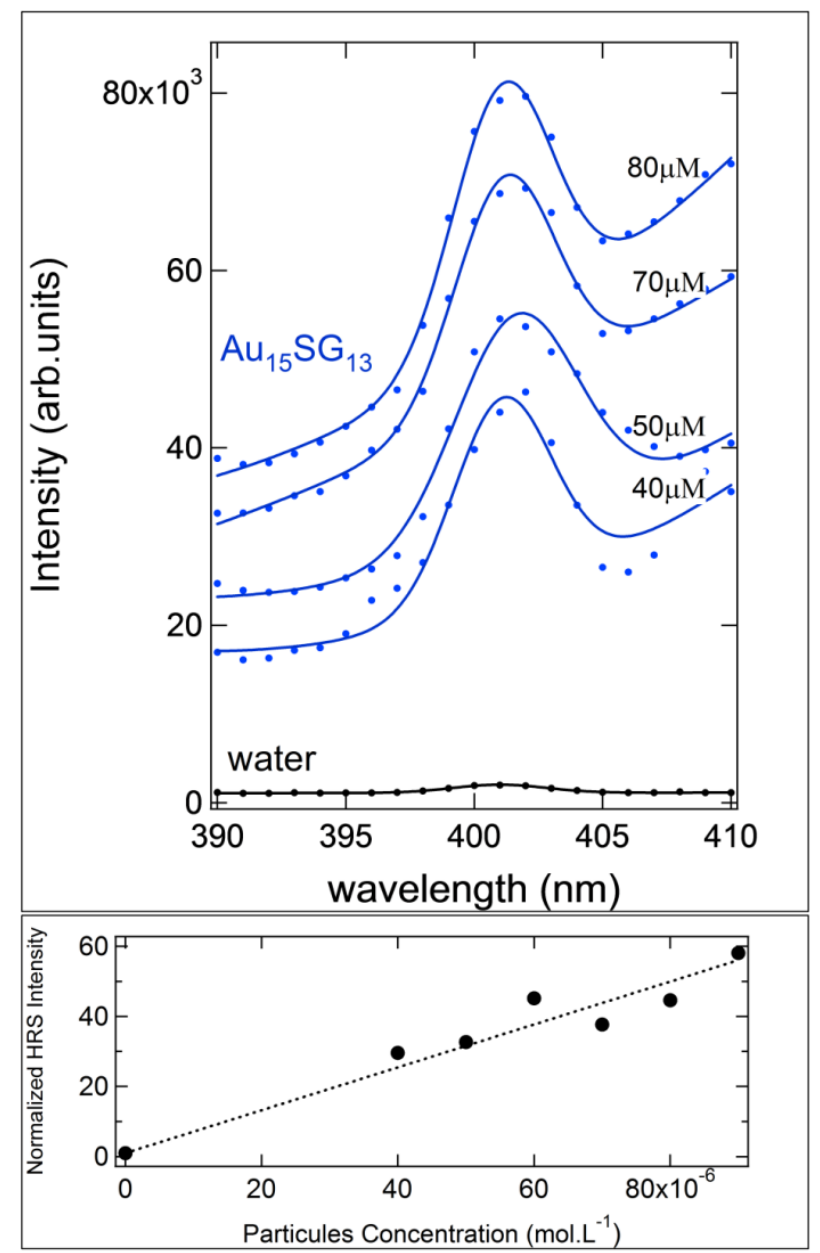

Figure 6.2 : (top panel) HRS intensity versus wavelength for Au15SG13 solutions of different concentrations (blue circles). Lines : fit to a Gaussian function superposed on a linearly increasing function of the wavelength. (bottom panel) Plot of the HRS intensity of for Au15SG13 as a function of concentration. The dotted line corresponds to the linear adjustment to Eq. 6.7. Adapted from Ref. ${ }^{[7]}$ 
The HRS process is effectively an incoherent nonlinear process requiring the conversion of two fundamental frequency photons into one harmonic frequency photon. For molecules that display TPEF, both HRS signal and two-photon excited photoluminescence are collected during the experiment. Two methods have been proposed in the past to distinguish Hyper Rayleigh Scattering and Two-Photon Excited Photoluminescence. The first one is a timedomain experiment where the fundamental beam is modulated at a varying frequency. This method has been proposed by the group of André Persoons in Leuven (Belgium) ${ }^{[4,8]}$ Because we run our experiment in a gated photon counting regime, this method is not well suited because of the instrumentation which is required for the low-level signal recorded. The second one has been proposed by us and relies on the spectral width of the HRS line determined by the fundamental laser spectral bandwidth in contrast to the photoluminescence bandwidth that is much broader. As a result, in the course of the experimental procedure, spectral analysis of the data is always performed and the broadband photoluminescence spectrum subtracted. Here, it is pointed out that HRS and photoluminescence are two incoherent processes and they can indeed be subtracted. This ensures that the data do not contain any spurious contributions from other processes.

\section{Two-photon absorption}

As described in chapter 3, two photon absorption (TPA) is a third order NLO process, and corresponds to the simultaneous absorption of two photons of the same energies (degenerate TPA) or different energies (non-degenerate TPA), in the presence of intense radiation. The degenerate TPA process is characterized by $\chi^{(3)}(-\omega ;-\omega, \omega, \omega)$. At sufficiently high intensities, the probability of a material absorbing more than one photon before relaxing to the ground state can be greatly enhanced. Two-photon absorption involves a transition from the ground 
state of a system to a higher-lying state by the simultaneous absorption of two photons from an incident radiation field.

The third order process in which the refractive index of the medium changes with respect to the intensity of the incident optical field and the total refractive index of such medium is given by

$n=n_{0}+n_{2} I$

where $n_{0}$ is the linear refractive index and $n_{2}$ is the field-dependent part. Depending on the sign of $n_{2}$, the laser beam inside the material experiences a focusing or a defocusing effect leading to the self-focusing or the self-defocusing phenomenon.

In the same way, the intensity-dependent absorption can be described in terms of linear and nonlinear absorption coefficients :

$\alpha=\alpha_{0}+\delta I$

where $\alpha_{0}$, and $\delta$ are, the linear absorption and third order nonlinear absorption coefficients, respectively.

\section{a) Z-scan technique}

Z-scan technique allowing for sensitive measurements is based on self-focusing effect. Since it is established, it has become a popular for characterizing third order nonlinear optical materials. This technique enables simultaneous measurement of nonlinear index of refraction $\mathrm{n}_{2}$, and nonlinear absorption coefficient $\delta$, which are related to the real and imaginary parts of third order nonlinear optical susceptibility $\chi^{(3)}$, respectively. Basically, a gaussian laser beam is focused using a lens on the sample and by translating the sample through the focus, the change in the far-field intensity pattern is monitored (see figure 6.3). Here, the sample is translated along the axis of the laser beam, taken to be the Z-axis, from the left to the right of 
the focal point. The transmitted intensity is first measured keeping an aperture in front of the detector thus obtaining closed aperture Z-scan data. The transmittance is measured without aperture since the sample is translated along the Z-direction in order to obtain the open aperture Z-scan data. The sample itself behaves as a thin lens for the laser beam propagating through it. When the sample is translated along the beam path, its effective focal length changes depending on the intensity of the converging laser beam. This results in a change of the intensity distribution in the far-field zone and is measured by the closed aperture Z-scan technique. In addition to this refraction due to nonlinearity, the medium also possesses nonlinear absorption, which is significant in the open aperture measurement. Thus, altogether the closed aperture and the open aperture allow us to calculate values of both $\mathrm{n}_{2}$ and $\delta$.
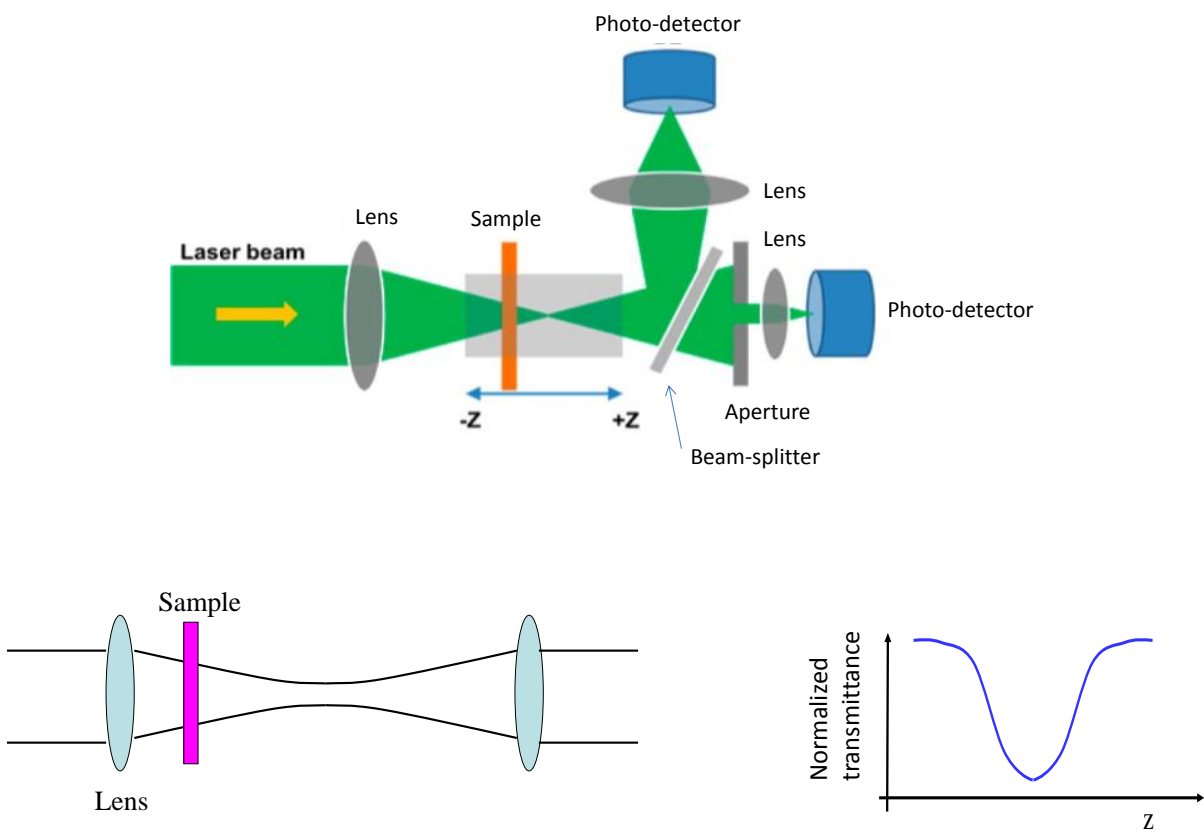

Open aperture Z-scan
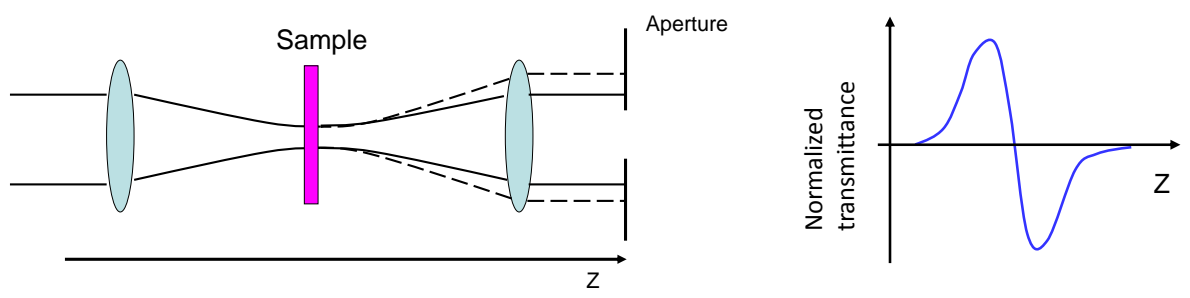

Closed aperture Z-scan

Figure 6.3: Schematic view of a classical z-scan set-up geometry. A gaussian laser beam is focused using a lens on the sample and by translating the sample through the focus, the change in the far-field intensity pattern is monitored. Open and Close aperture Z-scan principles, along with corresponding transmittance as a function of distance Z, are also presented. 
A model to describe the nonlinear absorption of a thin sample probed with a moderate intensity Gaussian beam has been proposed by Sheik-Bahae et al. ${ }^{[9]}$ The normalized transmittance can be expressed in terms of the peak irradiance in a summation form more suitable for numerical evaluation:

$T(z)=\frac{\sum_{m=0}^{\infty}\left[\frac{-\delta I_{0} L_{e f f}}{1+\left(\frac{z}{z_{0}}\right)^{2}}\right]^{m}}{(m+1)^{3 / 2}}$

In this equation, $z_{0}$ is the diffraction length and $I_{0}$ is the on-axis intensity at the beam waist $(\mathrm{z}=0)$. One should note that Eq. (6.10) is valid only for $\delta I_{0} L_{e f f}<1$ or otherwise the series diverges. The effective length $L_{\text {eff }}$ is used to correct the intensity decrease along the depth of the sample due to linear absorption: $L_{e f f}=\frac{1-e^{-\alpha L}}{\alpha}$. (where a is linear absorption coefficient). For weak linear absorption, $L_{\text {eff }}$ may be replaced by the physical sample thickness $L$.

For metal nanoclusters, some femtosecond z-scan measurements have been reported and were performed by using a mode-locked Ti:sapphire oscillator seeded regenerative amplifier, which gives outcoming laser pulses with a central wavelength at $800 \mathrm{~nm}$ and a repetition rate of 1 $\mathrm{kHz}$. The laser beam was focused onto the gold cluster solution $\left(1 \times 10^{-6} \mathrm{M}\right)$ samples contained in a $1 \mathrm{~mm}$ thick quartz cuvette, with a beam waist of $\sim 20 \mu \mathrm{m}$. The duration of the laser pulse at the sample is $\sim 200 \mathrm{fs}$. The sample was moved along the $\mathrm{z}$ axis towards and away from the focus point while the transmitted signal was monitored with a lock-in amplifier The TPA coefficient $(\delta$ ) (in units of $\mathrm{cm} / \mathrm{GW}$ ) was obtained by fitting the Z-scan data with a two-photon absorption model and then the TPA cross section $\left(\sigma_{2}\right)$ (in units of $1 \mathrm{GM}=10^{-50}$ $\mathrm{cm}^{4} \mathrm{~s}$ /photon molecule) was calculated by using the following equation: 
$\sigma_{2}=\frac{\delta h v \times 10^{3}}{N_{A} c}$

where $N_{A}$ is the Avogadro constant, $h$ is the plank constant, $c$ is the concentration of the gold cluster solution and $v$ is the frequency of the incident laser. Figure 6.4 shows the z-scan measurement results using $800 \mathrm{~nm}$ femtosecond laser pulses as the excitation source with a pulse energy of $180 \mathrm{~nJ}$, glutathione monolayer protected gold clusters by using a z-scan technique (open aperture configuration). The data can be well fit using a two-photon absorption model (described above) and the TPA cross section of the gold clusters was calculated to be $\sim 189740 \mathrm{GM} .^{[10]}$

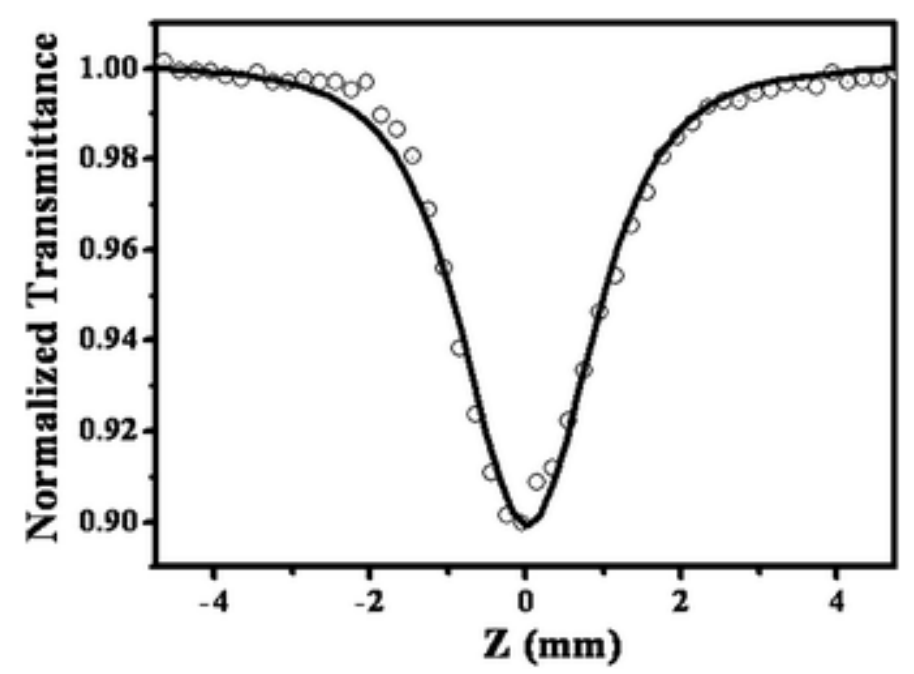

Figure 6.4 : z-Scan measurement results on the gold clusters under femtosecond laser excitation at $800 \mathrm{~nm}$. Adapted from ref. ${ }^{[10]}$

\section{b) P-scan measurements}

Nonlinear absorption can also be probed using an alternative technique named P-scan (or Power-scan) during which the incident power is directly varied and the induced changes in the sample transmission are measured. ${ }^{[11]}$ For small nonlinear effects, the absorption coefficients 
expansion can be reduced to Eq. (6.9). The transmission factor $T$ of the sample can then be expressed as a function of intensity using the standard Beer-Lambert law:

$T=C e^{-\alpha L}=C e^{-(\alpha+\delta I) L}$

where $C$ is a constant. If the factor $\delta I_{0} L_{e f f}$ is small compared to unity, it is possible to approximate the transmission with the following expression:

$T(I) \approx T(0)-T(0) \delta L_{e f f} I$

where $T(0)$ is a constant depending on $C$ and on the linear transmission $e^{-\alpha L}$. The beam onaxis intensity is expressed as a function of the pulse power $P_{m}$ measured before the sample:

$I=\frac{(1-R) P_{m}}{\pi \omega_{0}^{2}}$

Here, $R$ is the sample front interface reflection coefficient and $\omega_{0}$ is the beam waist. Thus, the transmission can be calculated as a function of the measured power as

$T(I) \approx T(0)-T(0) \delta L_{e f f} \frac{(1-R) P_{m}}{\pi \omega_{0}^{2}}$

indicating a linear dependence between the measured power and the sample transmission. It is possible to extract $\delta$ from the slope of the $T\left(P_{m}\right)$ vs. $P_{m}$ dependence. 


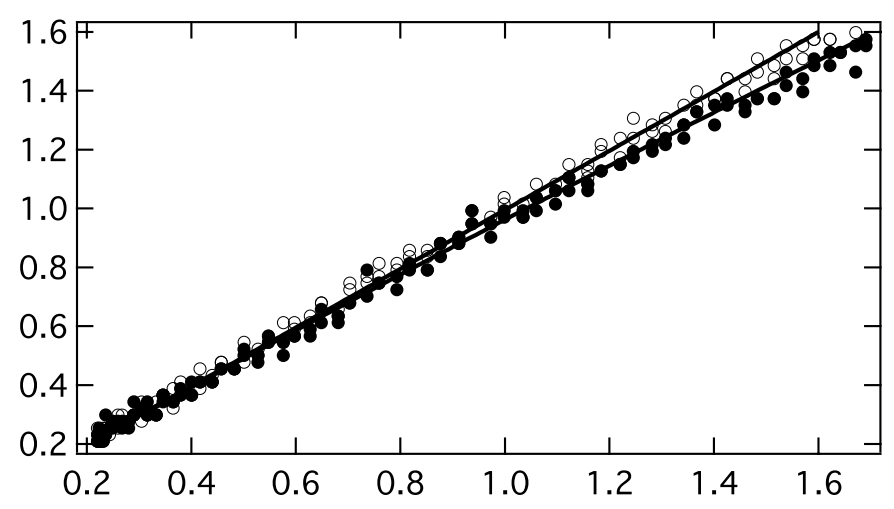

Figure 6.5 : Transmitted intensity recorded at $780 \mathrm{~nm}$ (empty circles) in absence of the cell, (filled circles) with the cell. Solid lines are fit using a nonlinear absorption.

The P-scan configuration offers at least two advantages over the traditional Z-scan measurements, namely it is less time consuming and is less sensitive to geometry issues (parallelism, reflections, dust, etc.).

For metal nanoclusters, we reported some femtosecond p-scan measurements ${ }^{[7]}$ using a modelocked femtosecond Ti:sapphire laser delivering at the fundamental wavelength of $780 \mathrm{~nm}$ pulses with a duration of about 140 femtoseconds at a repetition rate of $76 \mathrm{MHz}$. The beam was gently focussed by a low NA microscope objective to a waist of few $\mu \mathrm{m}$ and sent in transmission into a $1 \mathrm{~cm}$ path length spectrophotometric cuvette. The transmitted light was detected with a large aperture photodiode. The incident power was controlled with a halfwave plate and a polarizing cube. A typical nonlinear absorption plot is shown in Figure 6.5. As expected, in absence of the cell, the plot exhibits a linear behavior with a slope of unity whereas in the presence of the cell, a decrease of the transmitted intensity is observed as the absorption increases due to a nonlinear contribution.

\section{c) Two-photon absorption photoluminescence (TPA PL) technique}

This last technique requires that molecules emit light upon 2-photon excitation. Two-photon absorption (TPA) spectra of the compounds studied are then measured using TPA 
photoluminescence (TPA PL) spectroscopy. ${ }^{[12]}$ Fluorescence is excited in samples via TPA by directing tightly collimated and high-intensity laser beam on a sample. The emission from the sample is collected at $90^{\circ}$ angle by a high numerical aperture lens and directed to a spectrometer's entrance slit. The spectrally integrated intensity of the fluorescence excited via TPA process is :[12]

$I=A \eta \int_{-\infty}^{+\infty} \frac{I_{0}^{2}(t) \sigma_{2} c l}{1+I_{0}(t) \sigma_{2} c l} d t$

Here, $\sigma_{2}$ is the TPA cross-section, $c$ the concentration of the molecules, $l$ the interaction length, and $\eta$ is the fluorescence efficiency. Factor $A$ includes all experimental parameters. Function $I_{0}(t)$ describes temporal profile of the excitation pulse. In case of the weak excitation regime, one can assume that $I_{0}(t) \sigma_{2} c l \ll 1$. Then equation 6.16 takes form:

$I=A \eta \sigma_{2} c l \int_{-\infty}^{+\infty} I_{0}^{2}(t) d t=B \eta \sigma_{2} c l P^{2}$

$B$ is a constant and $P$ is the excitation light power. Since $B$ is not trivial to determine, relative measurements technique using reference sample with known TPA spectrum are employed. In this case, the ratio of the integrated PL intensities for the reference and studied samples can be expressed and one can easily find the TPA cross-section of the compound studied:

$\sigma_{2}=\frac{\eta^{r e f} \sigma_{2}^{r e f} c^{r e f} P_{r e f}{ }^{2}}{\eta c P^{2}} \frac{I}{I^{r e f}} K$

Here, the index ref denotes values related to the reference measurements. $\mathrm{K}$ is a correction factor taking into account the difference in refractive indices of the studied and the reference 
material. For reference materials, coumarin, fluorescein, and rhodamine laser dyes have been usually used.

\section{Two-photon excited fluorescence cross section measurements}

These measurements are directly connected to Two-photon absorption photoluminescence (TPA PL) technique. The two-photon excited fluorescence cross section, $\sigma_{T P E F}$ is simply given by : $\sigma_{T P E F}=\eta \sigma_{2}$. If the measurements are performed on the same set-up at two close time intervals (i.e. we assume that the power fluctuates in the same manner during the two experimental periods of measurements), and with the same solvent, then Eq.( 6.18) can be transformed into :

$\sigma_{T P E F}=\frac{\eta^{r e f} \sigma_{2}^{r e f} c^{r e f}}{c} \frac{I}{I^{r e f}}$

Experimentally, we observe the following results when comparing the TPE fluorescence of the gold nanoclusters and fluorescein solutions, see Figure 6.6. 


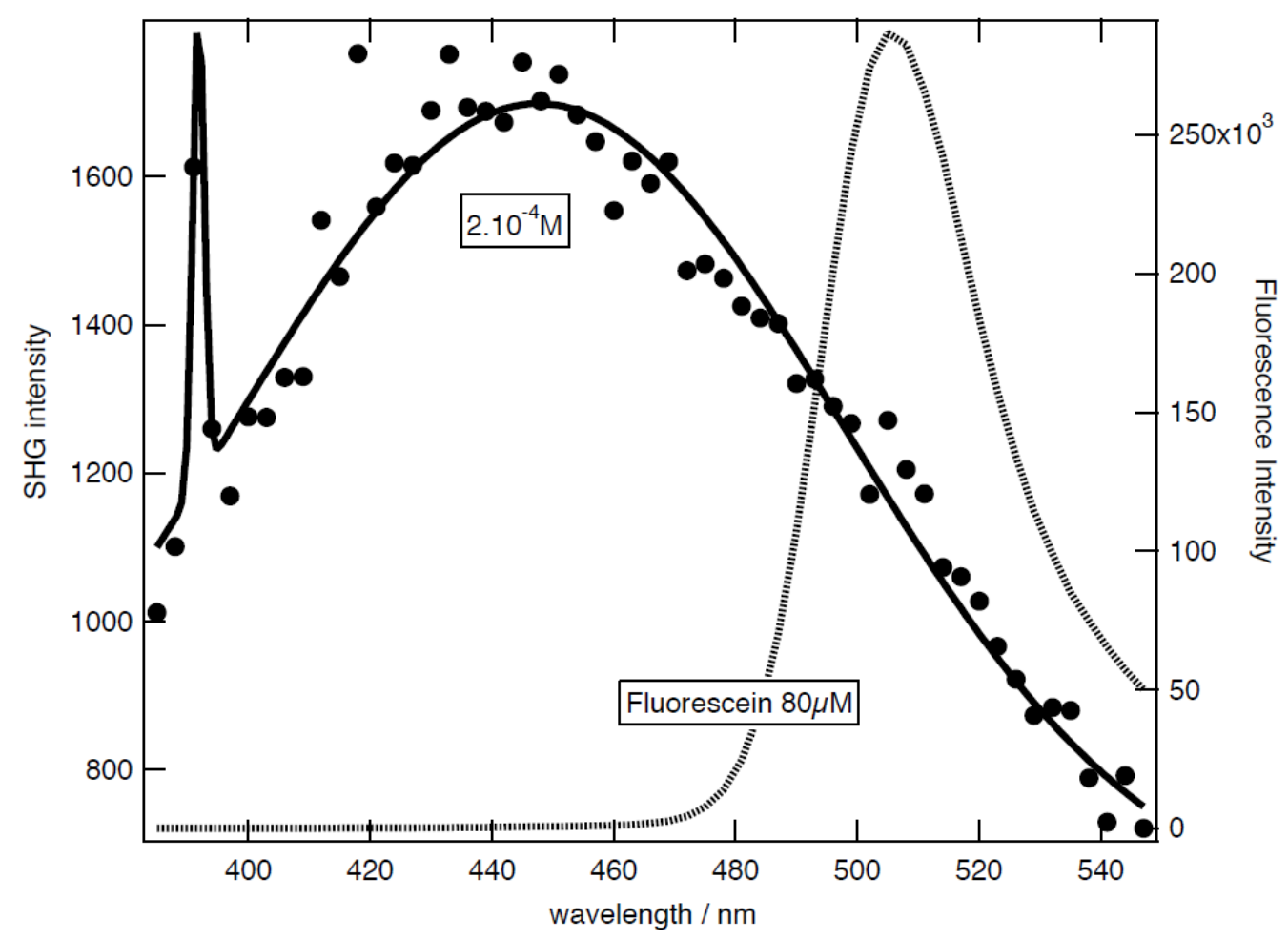

Figure

Figure 6.6 : TPE Fluorescence from aqueous solutions of (filled circles) $200 \mu \mathrm{M}$ glutathione protected gold nanoclusters and (Dotted) $80 \mu \mathrm{M}$ Fluorescein.

Figure 6.6 shows that the hyper Rayleigh scattering signal is easily observed at $392 \mathrm{~nm}$. This signal arises from the nonlinear scattering of the incident laser light impinging into the sample at a wavelength of $784 \mathrm{~nm}$. Here, we have chosen fluorescein dye as an internal reference. From the literature, we assume at the excitation wavelength of $780 \mathrm{~nm}$ : $\sigma_{2}($ fluorescein $)=33.3$ GM using a quantum yield of 0.9 and a two photon absorption cross-section of 37 GM. ${ }^{[13]}$ Fluorescein dye was used at a low concentration of $80 \mu \mathrm{M}$ in order to obtain two-photon emission yields compatible with the gold nanocluster two-photon yields.

To confirm that indeed a two--photon excited process was observed, a power dependence of the collected gold nanocluster two--photon excited fluorescence was measured. The square power dependence of the fluorescence intensity is verified as seen on Figure 6.7. 


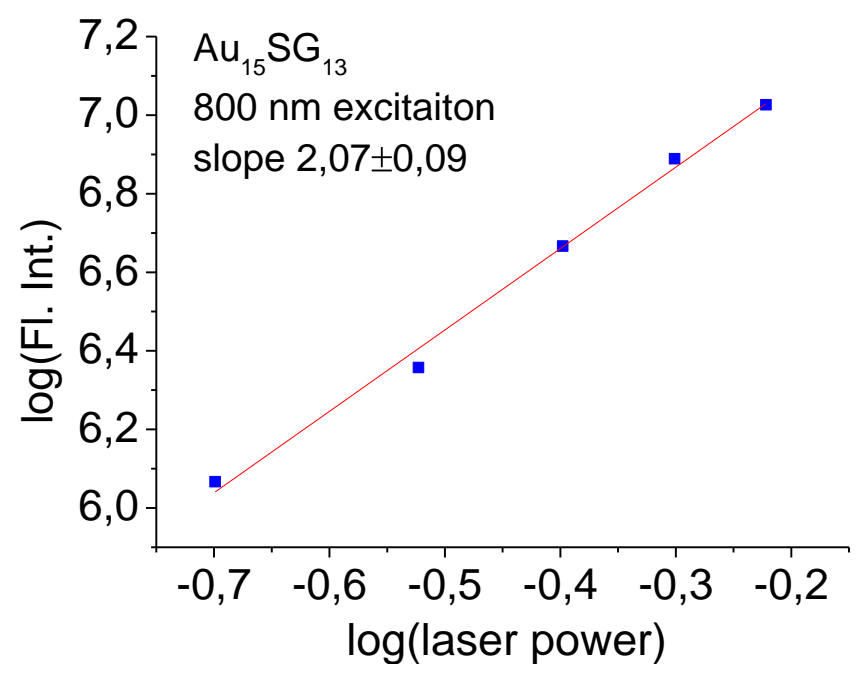

Figure 6.7 : Power dependence of emission spectra at different pump powers for glutathione protected gold nanoclusters after excitation at $800 \mathrm{~nm}$.

\section{Conclusion}

Based on the current state-of-the-art of experimental set-ups for the measurement techniques of optical nonlinearities, our experimental has proposed a "tout-en-un" experimental set-up able to record TPA, TPEF cross sections along with the first hyperpolarizability values for liganded silver and gold quantum clusters. The P-scan technique for TPA measurements has been preferred to the more conventional Z-scan technique because the P-scan configuration it is less time consuming and is less sensitive to geometry issues (parallelism, reflections, dust, etc.). In our experimental set-up, both hyper Rayleigh scattering and two-photon excited photoluminescence are recorded with the same detection line. We have proposed a simple method to distinguish this two NLO processes, and relies on the "separation" of spectral width of the HRS line determined by the fundamental laser spectral bandwidth in contrast to the photoluminescence bandwidth that is much broader. The current set-up provides accurate NLO cross sections and hyperpolarizability values with precision better than $10 \%$. 


\section{References.}

[1] B. F. Levine, C. G. Bethea. Second and third order hyperpolarizabilities of organic molecules. The Journal of Chemical Physics 1975, 63, 2666.

[2] J. L. Oudar. Optical nonlinearities of conjugated molecules. Stilbene derivatives and highly polar aromatic compounds. J. Chem. Phys. 1977, 67, 446.

[3] K. Clays, A. Persoons. Hyper-Rayleigh scattering in solution. Phys. Rev. Lett. 1991, 66, 2980.

[4] K. Clays, A. Persoons, Hyper-Rayleigh scattering. Opportunities for molecular, supramolecular, and device characterization by incoherent 2nd-order nonlinear light scattering, Vol. 9, Academic Press, 2001.

[5] P. C. Ray. Size and Shape Dependent Second Order Nonlinear Optical Properties of Nanomaterials and Their Application in Biological and Chemical Sensing. Chemical Reviews 2010, 110, 5332.

[6] J. Duboisset, G. Matar, I. Russier-Antoine, E. Benichou, G. Bachelier, C. Jonin, D. Ficheux, F. Besson, P. F. Brevet. First Hyperpolarizability of the Natural Aromatic Amino Acids Tryptophan, Tyrosine, and Phenylalanine and the Tripeptide Lysine-Tryptophan-Lysine Determined by Hyper-Rayleigh Scattering. The Journal of Physical Chemistry B 2010, 114, 13861.

[7] I. Russier-Antoine, F. Bertorelle, M. Vojkovic, D. Rayane, E. Salmon, C. Jonin, P. Dugourd, R. Antoine, P.-F. Brevet. Non-linear optical properties of gold quantum clusters. The smaller the better. Nanoscale 2014, 6, 13572.

[8] O. F. J. Noordman, N. F. van Hulst. Time-resolved hyper-Rayleigh scattering: measuring first hyperpolarizabilities $\beta$ of fluorescent molecules. Chemical Physics Letters 1996, 253, 145.

[9] M. Sheik-Bahae, A. A. Said, T. H. Wei, D. J. Hagan, E. W. V. Stryland. Sensitive measurement of optical nonlinearities using a single beam. IEEE Journal of Quantum Electronics 1990, 26, 760.

[10] L. Polavarapu, M. Manna, Q.-H. Xu. Biocompatible glutathione capped gold clusters as oneand two-photon excitation fluorescence contrast agents for live cells imaging. Nanoscale 2011, 3, 429.

[11] J.-M. Lamarre, F. Billard, C. H. Kerboua, M. Lequime, S. Roorda, L. Martinu. Anisotropic nonlinear optical absorption of gold nanorods in a silica matrix. Optics Communications 2008, $281,331$.

[12] C. Xu, W. W. Webb. Measurement of two-photon excitation cross sections of molecular fluorophores with data from 690 to $1050 \mathrm{~nm}$. JOSA B 1996, 13, 481.

[13] M. A. Albota, C. Xu, W. W. Webb. Two-Photon Fluorescence Excitation Cross Sections of Biomolecular Probes from 690 to $960 \mathrm{~nm}$. Applied Optics, 1998, 37, 7352. 


\section{Chapter 7}

\section{Selected studied cases.}

\section{Introduction.}

After discussing the theoretical and experimental methodology in order to investigate optical nonlinearities of liganded metal quantum clusters, we wish to present the various concepts useful for applications and illustrate them on some examples. The incentive is to demonstrate, by selected studied cases, how liganded gold and silver quantum clusters can serve to evaluate their nonlinear optical properties as a function of their size and composition. Liganded gold and silver quantum clusters, also termed as nanoclusters (NCs) have emerged as fascinating fluorescent nanomaterials and attracted considerable research interest in both fundamental research and practical applications, including biosensing, ${ }^{[1]}$ bioimaging, ${ }^{[2]}$ catalysis, and photonics. ${ }^{[3]}$ Different scaffolds used as stabilizer for nanoclusters have been proposed and were found to play a key role in their emissive properties. ${ }^{[4,5]}$ In a first part of this chapter, we investigate the emissive properties after both one- and two-photon excitation, i.e. one-photon and two-photon excited fluorescence spectra of silver clusters capped with different scaffolds. 
The comparison of their linear and non-linear optical properties revealed different selection rules for one-photon and two-photon processes. In addition, the second-harmonic generation requires the absence of centro-symmetry in the cluster, and in particular chiral clusters (intrinsically chiral or with chiral ligands) would lead to enhanced SHG efficiencies. The influence on the SHG properties of size, ligand composition (e.g., chiral ones) and core composition (e.g. silver/gold doping) will be discussed in the last section.

\section{Protected silver nanoclusters : Playing with colors and photons}

In this section we present a detailed study on the luminescent properties of ligated silver nanoclusters excited by one- and two photons. As a first example, we would like to focus on silver clusters capped with dihydrolipoic acid (DHLA), a ligand which binds very strongly to the silver atoms. 


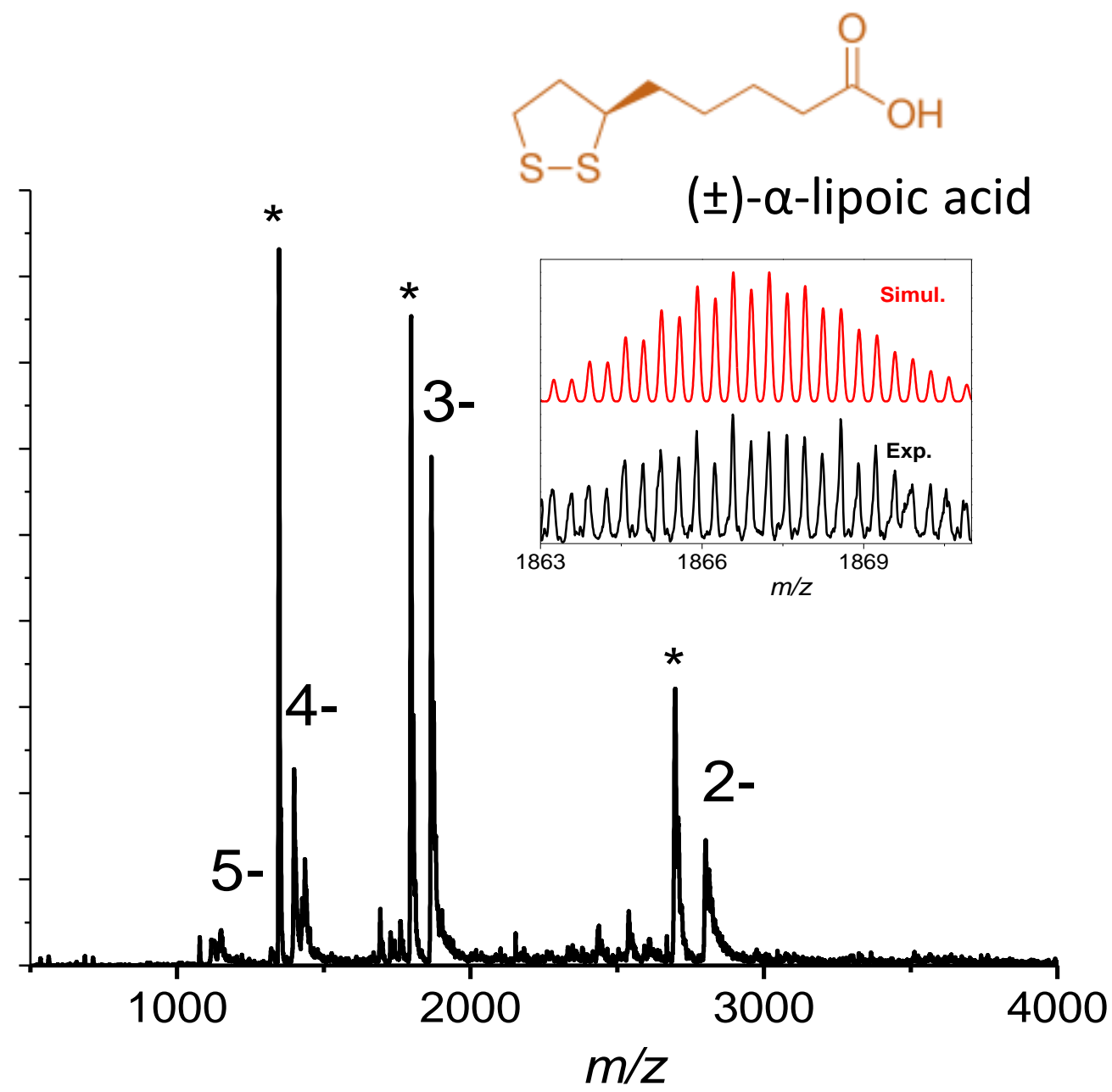

Figure 7.1 : ESI mass spectrum of the Ag clusters synthesized. The asterisk corresponds to peaks assigned to loss of one DHLA molecule from the Ag clusters inset: comparison of the experimental and simulated isotopic distributions for the 3 charge state of the $\mathrm{Ag}$ clusters $\left(\left[\mathrm{Ag}_{29}(\mathrm{DHLA})_{12}-3 \mathrm{H}\right]^{3-}\right)$ and structure of lipoic acid. A charge state distribution was observed ranging from $[\mathrm{M}-2 \mathrm{H}]^{2-}$ to $[\mathrm{M}-5 \mathrm{H}]^{5-}$ along with peaks at lower masses corresponding to the neutral loss of $208 \mathrm{Da}$ (one lipoic acid molecule). The ESI spectra in the inset show the isotopic resolution for the 3- charge state. Adapted from ref. [6]

The ligated silver clusters synthesis is based on the method already published by Adhikari et al. ${ }^{[7]}$ However, in order to avoid sodium adducts, $\mathrm{NaBH}_{4}$ and $\mathrm{NaOH}$ were replaced by tetramethylammonium borohydride $\left(\mathrm{TMABH}_{4}\right)$ and $\mathrm{NH}_{4} \mathrm{OH}$ respectively. ${ }^{[6]}$ Mass spectrometry was used to determine the cluster composition which corresponds to $\operatorname{Ag}_{29}($ DHLA) 12 , as shown in Fig.7.1. Concerning their structural characterization, the poor stability of water soluble DHLA monolayer protected silver clusters, has prevented it from any type of crystallization studies. Although the metal core system is stable, the nature of DHLA ligand plays an important role in its stability. Exposure to light and oxygen (opening 
the vial often) has a negative effect on sample stability, with these samples showing the shortest degradation times of just a few months. ${ }^{[8]}$

Insight from theory concerning structural and optical properties of ultrasmall silver NCs permits us to better address the nature of the metal core and the staple motifs that play a key role for NLO properties. Structures of $\mathrm{Ag}_{29}(\mathrm{DHLA})_{12}$ are composed from DHLA ligands in which two sulfur atoms are bound to different number of Ag atoms, such that silver cores of different sizes can be formed. In the case that the central Ag atom is present, the symmetric structure (point group $\mathrm{T}$ ) is formed. However, in the case of the neutral species, degenerate orbitals are not fully occupied due to odd number of electrons. For the structure with central $\mathrm{Ag}$ atom, two sulfur atoms of DHLA are bound to two and three Ag atoms respectively, forming $\mathrm{Ag}_{13}$ centered icosahedral structure in which twelve $\mathrm{Ag}$ atoms are bound to twelve $\mathrm{S}$ atoms (cf. Fig. 7.2 (left panel).

In contrast, if the central atom is not present, other structural classes arise. For example, the core of six $\mathrm{Ag}$ atoms as capped trigonal bipyramid can be formed, in which $\mathrm{S}$ atoms from DHLA are not bound to Ag atoms of the core. In this case, the high symmetry of structure is removed. Each $\mathrm{S}$ atom is bound to one or two $\mathrm{Ag}$ atoms not belonging to the core, as illustrated on Fig. 7.2 (right panel). In this case the interface between the silver core and the ligand staples ( $\mathrm{S}-\mathrm{Ag}-\mathrm{S})$ contains also silver atoms forming an additional ligand shell. 


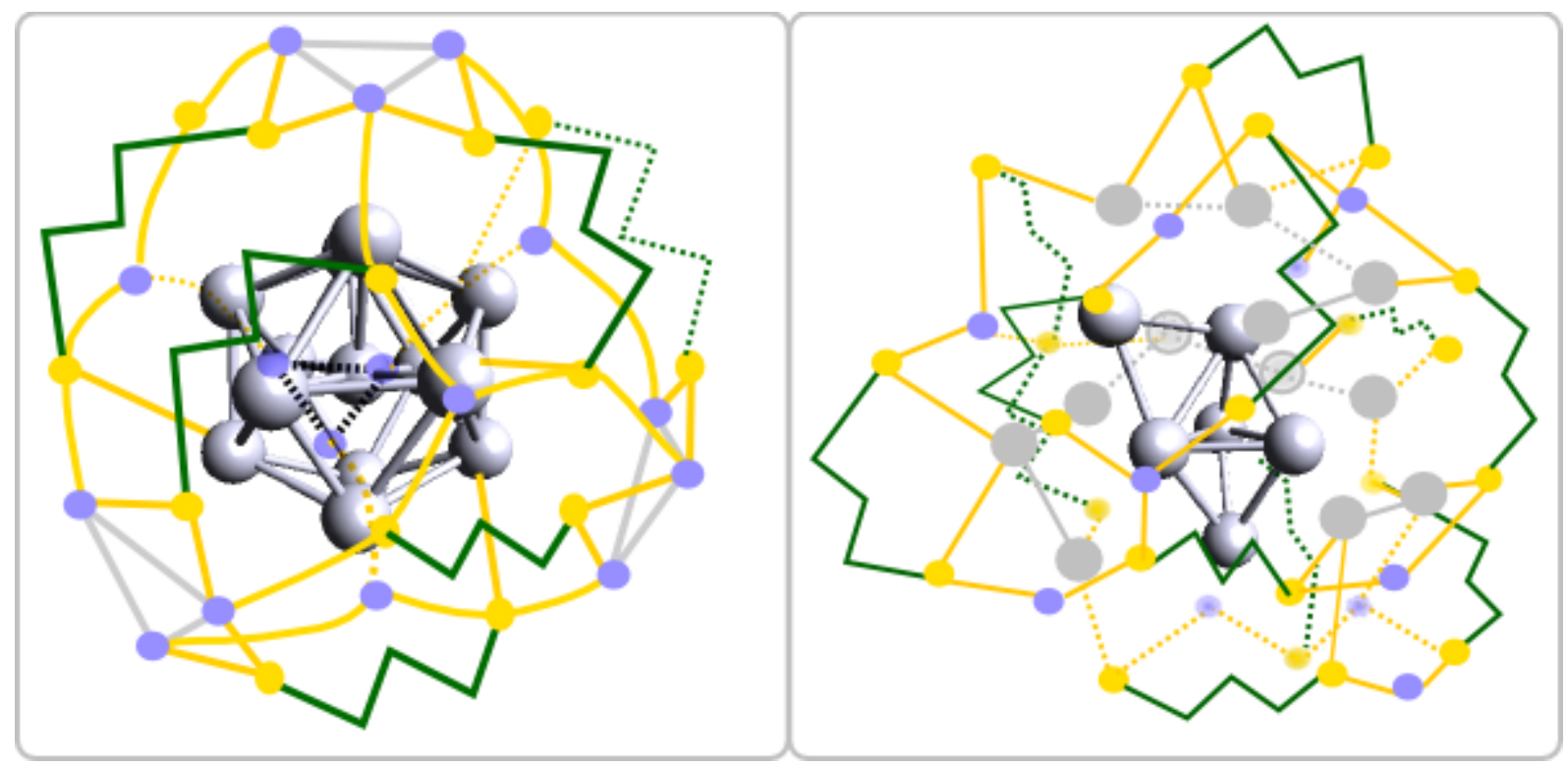

Figure 7.2: Illustrative presentation of DFT optimized structures of $\operatorname{Ag}_{29}(\mathrm{DHLA})_{12}$ showing two different structural classes. The DHLA is represented by $\mathrm{S}-\left(\mathrm{CH}_{2}\right)_{3}-\mathrm{S}$ and carboxylic tail is replaced by one $\mathrm{H}$ atom. On the left hand side: the central silver atom and twelve silver atoms are labelled by grey balls bound to twelve $S$ atoms (yellow balls) forming highly symmetrical subunit. Additional silver atoms involved in staples S-Ag-S are labelled by blue spheres, while green lines denote $3 \mathrm{CH}_{2}$ groups. On the right hand side: the grey balls label core of $6 \mathrm{Ag}$ atoms, grey circles label twelve $\mathrm{Ag}$ atoms which build ligand shell as interface between core and S atoms involved in staples S-Ag-S, while green lines indicate $3 \mathrm{CH}_{2}$ groups. The dashed lines are introduced to visualize the third dimension.

The optical properties of the Ag clusters were characterized in the UV-near IR range. The absorption spectrum in figure 7.3 shows several sharp transition bands with maxima at 328 , 428 and $499 \mathrm{~nm}$ combined with a shallow absorbance onset that starts around $600 \mathrm{~nm}$. Photoluminescence spectrum of dilute Ag nanoclusters in colloidal solution at room temperature is also presented in Fig. 7.3. The photoluminescence spectrum shows emission maxima at $\sim 670 \mathrm{~nm}$ upon excitation at $498 \mathrm{~nm}$. No significant change in emission maximum was observed by altering the excitation wavelength. The luminescence quantum yield is measured to be $2 \pm 1 \%$. PL lifetime of these Ag nanoclusters was measured in this study and a long lifetime contribution was found to be $\sim 1 \mu$ s. 


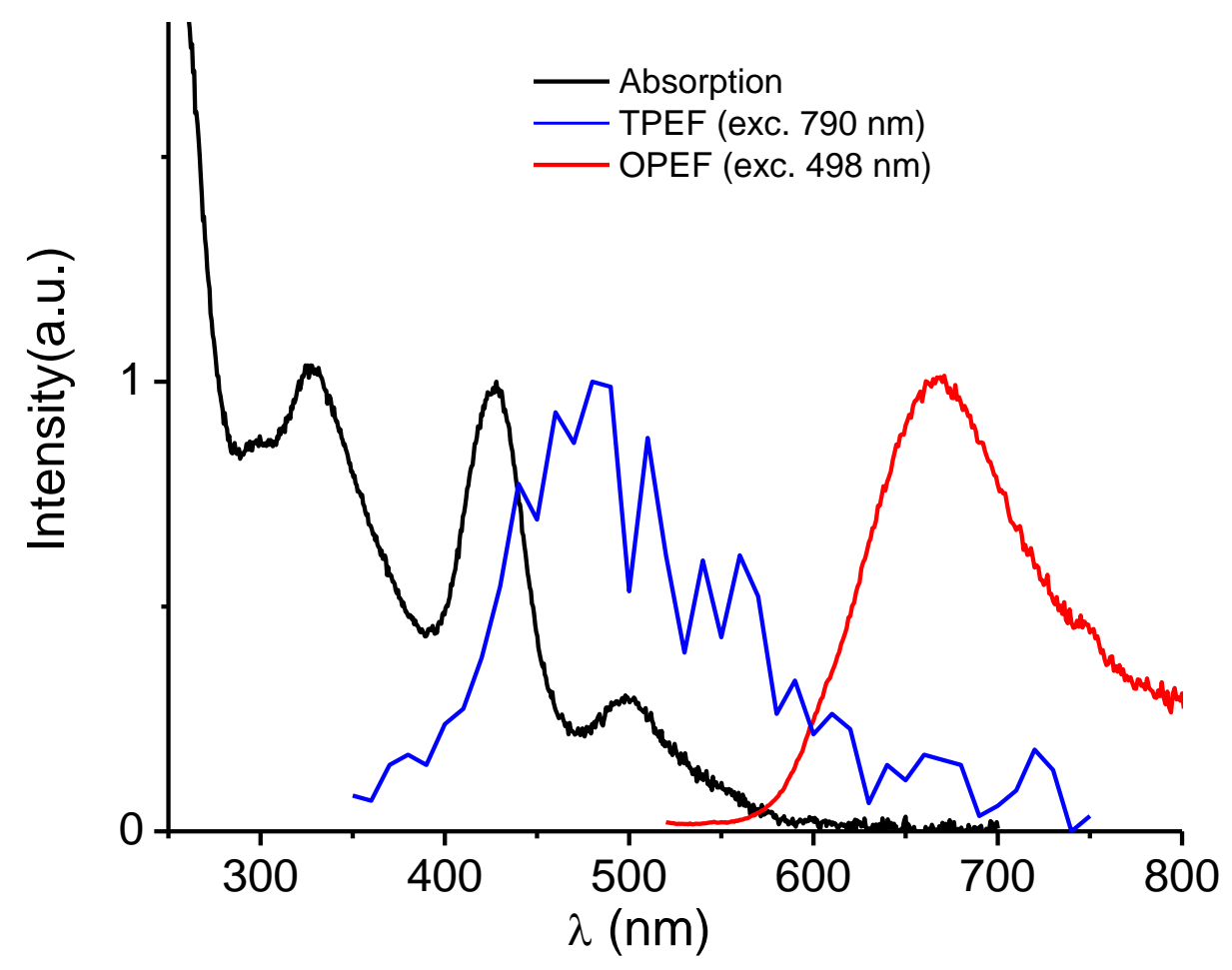

Figure 7.3: Spectroscopic characterization of the synthesized $\mathrm{Ag}_{29}(\mathrm{DHLA})_{12}$ clusters dispersed in water. Absorption spectrum (black), OPEF spectrum (red) and TPEF spectrum (blue). Adapted from ref. [6]

Two-photon excited fluorescence (TPEF) spectrum with excitations at $790 \mathrm{~nm}$ has been recorded for $\mathrm{Ag}_{29}$ clusters and is given in Fig. 7.3. A broad band in the visible range (between 400 and $650 \mathrm{~nm}$ ) is observed upon excitation at $780 \mathrm{~nm}$. The two-photon absorption and emission cross-section of silver nanoclusters, were determined using the methods reported in chapter 6. Experimentally, the TPE fluorescence cross section at $780 \mathrm{~nm}$ was $0.5 \mathrm{GM}$ and TPA cross section was $~ 50000 \mathrm{GM}$ for $\mathrm{Ag}_{29}(\mathrm{DHLA})_{12}$. These experimental cross-section values lead to quantum yield $\left(\mathrm{QY}=\sigma_{\mathrm{TPE}} / \sigma_{\mathrm{TPA}}\right)$ for $\mathrm{Ag}_{29}$ nanoclusters of $\sim 10^{-6}$. As also usually observed for gold nanoclusters, ${ }^{[9,10]}$ such protected silver clusters are excellent two-photon absorbers but present limited two-photon emission cross sections. TPEF spectra drastically differ from linear photoluminescence spectra where the red emission is observed (see Fig. 
7.3). Clearly, a tuning of emission from red to blue is triggered by the absorption of one or two photons.

Such difference between emission resulting from absorption of one or two photons was already reported by Dickson and coworkers ${ }^{[11]}$ on DNA-protected silver clusters. The excitation spectra of oligonucleotide-encapsulated clusters, with undefined number of $\mathrm{Ag}$ atoms, are blue-shifted with respect to the OPE peak, indicating that TPE accesses a higher excited electronic state than does OPE (Figure 7.4). The OPE excitation spectra at $620 \mathrm{~nm}$ $(\operatorname{Ag}[620 \mathrm{~nm}])$ and at $680 \mathrm{~nm}(\operatorname{Ag}[680 \mathrm{~nm}])$ show, in addition to the primary electronic transitions beyond $500 \mathrm{~nm}$, much less efficient excitation at higher energy which overlap with the doubled TPE transition energy. The relative transition strengths for OPE and TPE are strongly symmetry dependent. The question can be raised whether particular allowed transitions provide insight into validity of conventional parity rules ( $\mathrm{g}-\mathrm{u}$ for OPE, $\mathrm{g}-\mathrm{g}$ for TPE) and therefore of the chromophore symmetry. For instance, a distinction is observed between more symmetric organic dyes, such as rhodamine B and Fluorescein, with those having no center of symmetry, such as Cascade Blue. ${ }^{[12]}$ The latter group shows TPE spectra nearly identical with OPE spectra, suggesting that the parity rules are relaxed. For the centrosymmetric group, however, the TPE spectra are significantly blue-shifted relative to the OPE spectra, indicating that the first excited state is not accessible by TPE, and the transition occurs to a state that is accessible by TPE. Therefore, the OPE and TPE transitions leading to fluorescence are orthogonal. Since the parity rules appear to be relevant, it suggests that they possess inversion symmetry. This is no surprise if one considers the fluorophore as a globular, several-atom silver cluster. 

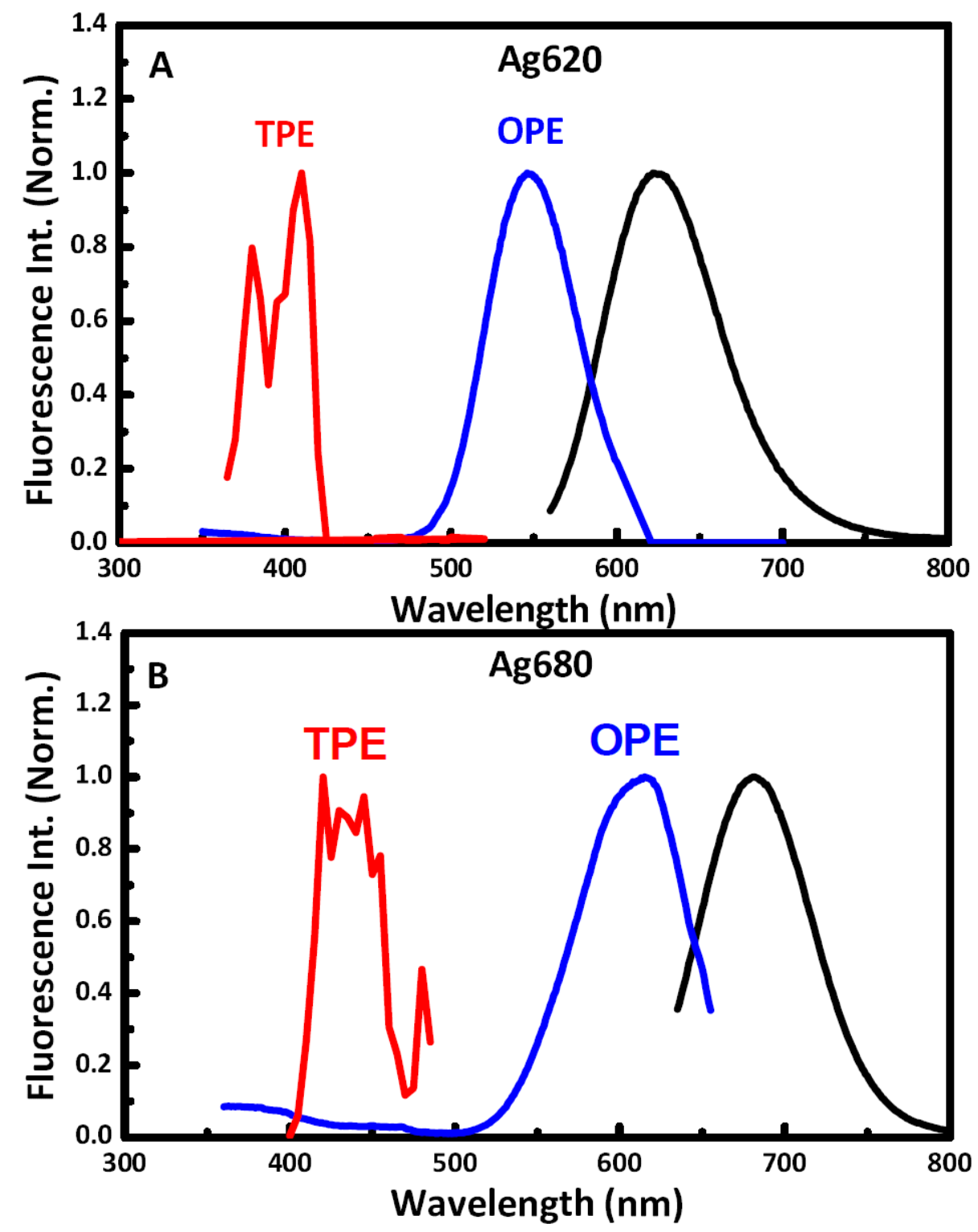

Figure 7.4: OPE and TPE (doubled in energy) spectra are shown along with the emission spectrum (black) for (A) $620 \mathrm{~nm}$ emitter and the (B) $680 \mathrm{~nm}$ emitter. Only a small portion of the OPE overlaps with the TPE spectrum (out as far as1040 $\mathrm{nm}$ ), but no overlap with the principal OPE peak at $540 \mathrm{~nm}$ is observed. In (B) there is overlap of the TPE peak to the weak OPE at around $430 \mathrm{~nm}$. The OPE and TPE curves are separately normalized to unity based on the emission output. Adapted from ref. ${ }^{[11]}$

Charge transfer induced emissions through ligand-to-metal or ligand-to-metal-metal (LMCT/LMMCT) always show a longer fluorescence lifetime, partly because LMCT/LMMCT can influence the excited state relaxation dynamics. Therefore, the observed emission is thought to arise from states containing $\operatorname{Ag}(\mathrm{I})$ atoms on the nanocluster surface. These states might be present as $\mathrm{Ag}(\mathrm{I})$-thiolate complexes capping a $\operatorname{Ag}(0)$ core, 
corresponding to structural feature that has been found for gold thiolate nanoclusters. ${ }^{[13]}$ The difference observed between the OPEF and TPEF spectra may therefore be accounted for the different symmetry rules required for the metal-to-metal excitations within the silver cluster core and the LMCT/LMMCT excitations as shown in the excitation pathway scheme given in Fig. 7.5.

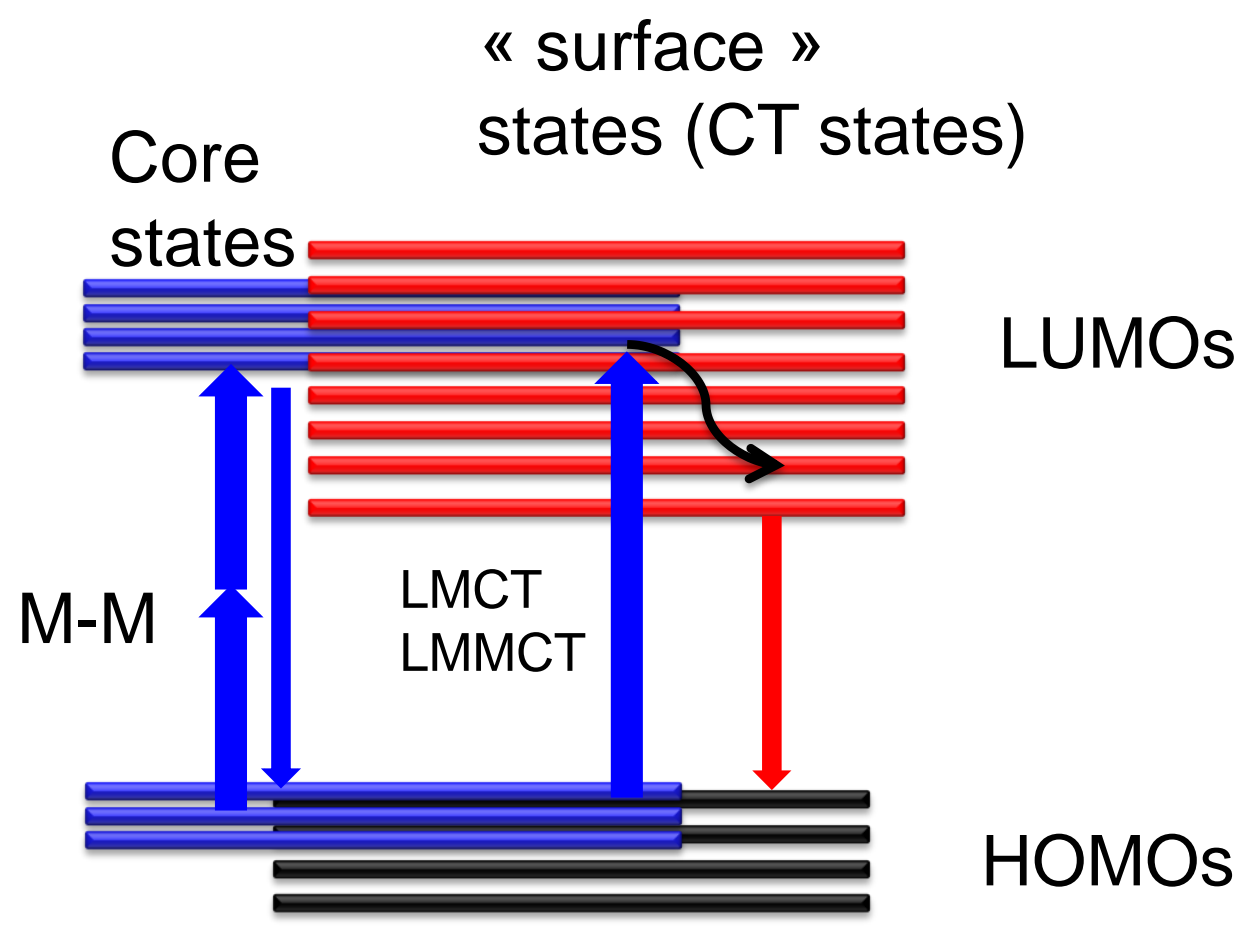

Figure 7.5 : Schematics showing the excited state relaxation dynamics in the $\mathrm{Ag}_{29}(\mathrm{DHLA})_{12}$ clusters in OPEF and TPEF experiments.

\section{Ligated gold nanoclusters: the quantum cluster regime.}

For gold and silver metal particles, their unique properties are mainly due to the collective excitation of the conduction band electrons known as the surface plasmon resonance (SPR). Due to the SPR properties, large magnitudes for the hyperpolarizability tensor of silver and gold nanoparticles have been reported. ${ }^{[14]}$ However, within the electric dipole approximation, for perfectly spherical metal nanoparticles, no HRS signal intensity should be collected 
owing to the centrosymmetry of the particle. For metal gold particles, it has been demonstrated that the harmonic generation finds its origin at the surface of the particles when the shape of the particles are not perfectly spherical. As a consequence, the total HRS response is of electric dipole nature for small particles; however, for larger particles, retardation effects in the electromagnetic fields must be considered, and a non-negligible quadrupolar contribution is observed. ${ }^{[15,16]}$ It has been demonstrated that a similar origin for the HRS process occurs for silver particles.

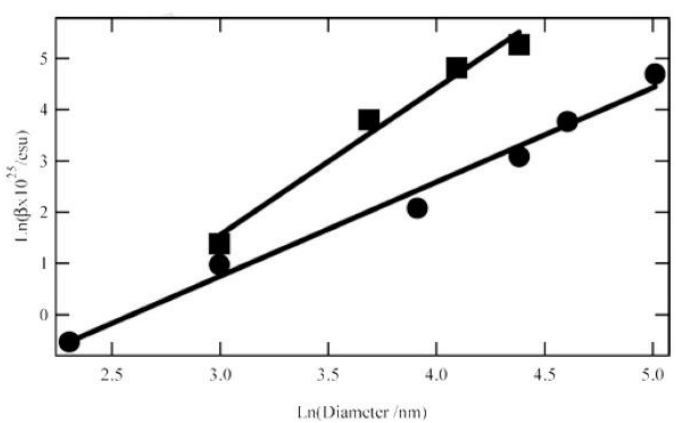

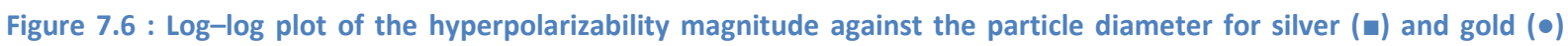
particles at the harmonic wavelength of 390 and $400 \mathrm{~nm}$, respectively (adapted from Ref. ${ }^{[17]}$ ).

Nappa et. al and Russier-Antoine et. al. ${ }^{[17]}$ reported the value of the hyperpolarizability for aqueous suspensions of silver metal particles, with diameter ranges from 20 up to $80 \mathrm{~nm}$, using $780 \mathrm{~nm}$ incident light. They have demonstrated that although the origin of the SHG arises from the surface of the particles, the size dependence of the $\mathrm{SH}$ intensity exhibits a scaling with the volume of the particles. Their results also show that the hyperpolarizability magnitude obtained for gold nanoparticles (at the fundamental wavelength of $800 \mathrm{~nm}$ ) is smaller than that of silver particles, due to the harmonic wavelength $(400 \mathrm{~nm})$ used in these experiments, which is in close vicinity with the silver SPR wavelength (see fig. 7.6).

However, what happens when the size of the noble metal particles decreases? 
In general, for small clusters a few nm size, the Mie-Drude model is no longer appropriate for quantitative interpretation of absorption spectra (see chapter 2). The optic response is described in terms of a molecular-like behavior of nanoclusters leading to an electronic energy quantization and the changes in the leading features of the patterns as a function of the cluster size.

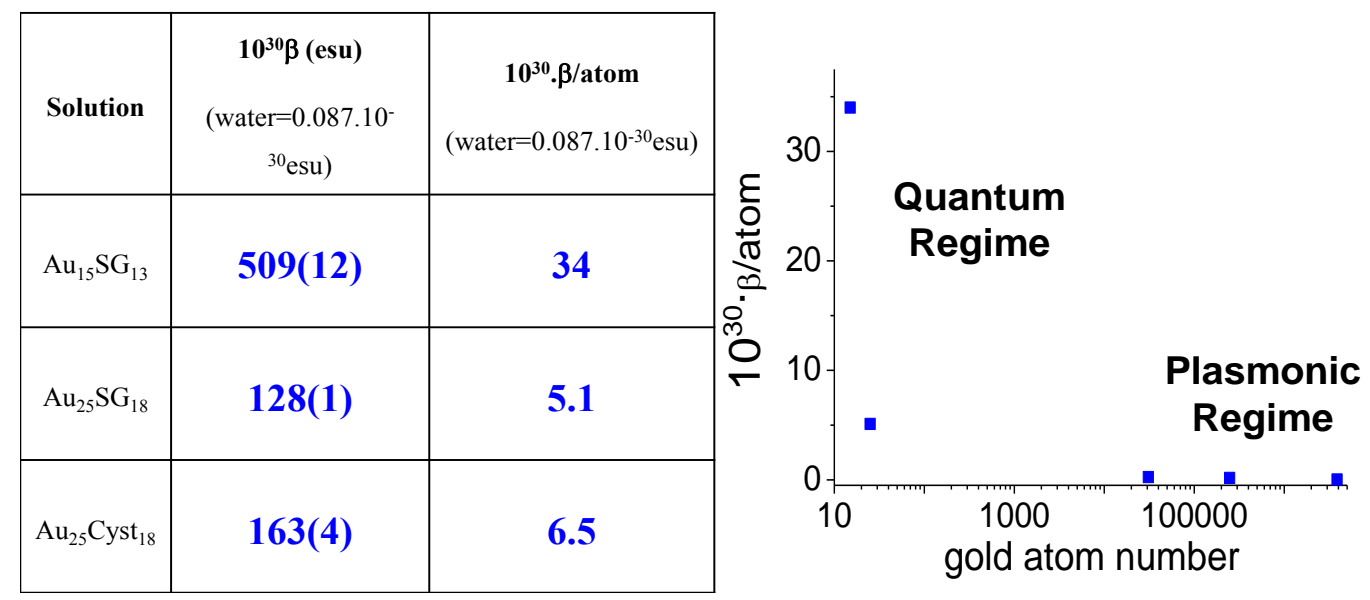

Figure 7.7 : (left) Quadratic hyperpolarizabilities for the different solutions at $802 \mathrm{~nm}$ (right) $10^{30} \beta$ per atom as a function of the number of gold atoms, obtained by $802 \mathrm{~nm}$ excitation. SG and Cyst for glutathione and cysteine ligands. Adapted from ref. ${ }^{[10]}$

The HRS intensity recorded for different concentrations of $\mathrm{Au}_{15} \mathrm{SG}_{13}, \mathrm{Au}_{25} \mathrm{SG}_{18}$ and $\mathrm{Au}_{25} \mathrm{Cyst}_{18}$ in aqueous solution was reported by Russier-Antoine et al., ${ }^{[10]}$ and permitted to determine the hyperpolarizability of these nanoclusters. The hyperpolarizability values obtained for the different solutions are reported in fig. 7.7. Interestingly $\mathrm{Au}_{25} \mathrm{SG}_{18}$ and $\mathrm{Au}_{25} \mathrm{Cyst}_{18}$ have similar hyperpolarizability, meaning that the ligand size has only a weak effect on the SH generation. An absolute value of $(0.16 \pm 0.01) \times 10^{-30}$ esu for the first hyperpolarizability of non-aromatic amino acids was reported by Duboisset et al. ${ }^{[18]}$ Using a collagen like model, the microscopic hyperpolarizability along the peptide bond was evaluated at $(0.7 \pm 0.1) \times 10^{-30} \mathrm{esu}$. It is clear that the huge hyperpolarizabilities reported in this work cannot be accounted by the ligand shell alone (which only would give a contribution of several $10^{-30}$ esu for the first hyperpolarizability for a fully coherent 
superposition). The first hyperpolarizability for ligated $\mathrm{Au}_{15}$ clusters, as compared to ligated $\mathrm{Au}_{25}$ clusters, is also larger considering the difference in the number of gold atoms. Structural theoretical investigations on the $\mathrm{Au}_{15}(\mathrm{SR})_{13}$ model feature staple motifs protecting the $\mathrm{Au}_{4}$ nucleus. ${ }^{[19,20]}$ On the other hand, the ligated $\mathrm{Au}_{25}$ cluster contains a centered icosahedral $\mathrm{Au}_{13}$ core capped by twelve gold atoms that are situated in six pairs around the three mutually perpendicular 2-fold axes of the icosahedron. ${ }^{[21]}$ Both ligated $\mathrm{Au}_{15}$ and $\mathrm{Au}_{25}$ structures are expected to have symmetric gold core structures, which exclude any intense SH generation. The nature of staple motifs protecting the gold core and in particular the structuration of $\mathrm{Au}-$ $\mathrm{S}$ bonds may be different between ligated $\mathrm{Au}_{15}$ and $\mathrm{Au}_{25}$ clusters, and may account for the enhanced SH signal for the former one.

Finally, we would like to emphasize that the $10^{30} \beta$ per atom values reported for ligated $\mathrm{Au}_{15}$ and $\mathrm{Au}_{25}$ clusters are more than two orders of magnitude larger than the values reported for $\mathrm{Au}$ NPs in the size range $10-50 \mathrm{~nm}$ (see Fig.7.7). $10^{30} \beta$ per atom values decrease drastically with increasing number of gold atoms and there is a saturation- type of behavior for large gold nanoparticles. The results outline the transition from the "molecular-like" cluster behavior to the nanoparticle behavior showing strong surface plasmon absorption.

\section{Ligated nanoclusters : playing with symmetry, size and composition.}

In this section, the influence on the NLO properties of size, ligand composition (e.g., chiral ones) and core composition (e.g. silver/gold doping) will be discussed on examples of selected studied cases.

We recently reported a facile "one-pot-one-size" synthesis of $\mathrm{Au}_{10}(\mathrm{SG})_{10} \mathrm{NCs}$ characterized by electrospray mass spectrometry (see fig. 7.8). ${ }^{[22]}$ The distinct X-ray diffraction pattern of $\mathrm{Au}_{10}(\mathrm{SG})_{10}$ was utilized as a signature for homoleptic gold-glutathione catenanes. The inset of 
Fig. 7.8a) shows the $\mathrm{CD}$ spectrum for $\mathrm{Au}_{10}(\mathrm{SG})_{10}$ in dilute aqueous solution along with its observed chiroptical effects resulting in positive and negative bands in the 250-400 nm.region. Our threoretical considerations showed that the origin of the chirality stems from the structure of mutually interacting S-Au-S bonds which are responsible for the leading features of the CD spectrum, with a small contribution from ligand asymmetry.
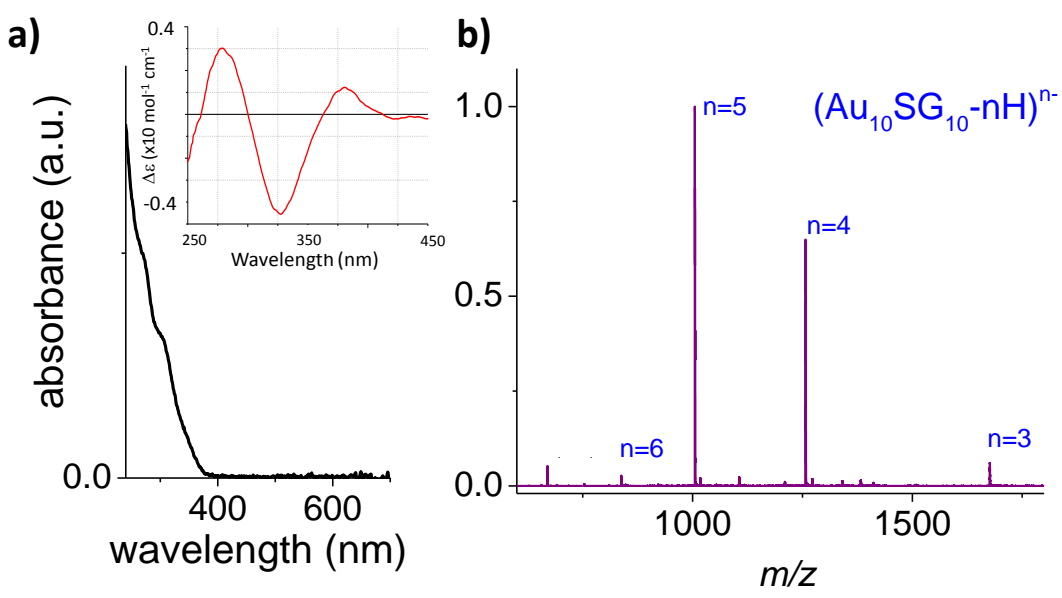

c)

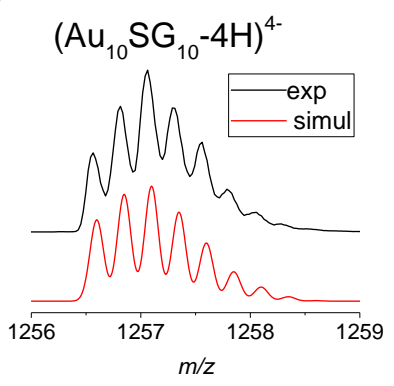

Figure 7.8 : a) UV-vis absorption and $C D$ spectrum (inset) of as-synthesized $A_{u_{10}}(S G)_{10} N C s$ in aqueous solution. (b and c) ESI mass spectrum of the as-synthesized $\mathrm{Au}_{10}(\mathrm{SG})_{10} \mathrm{NCs}$ and experimental and simulated isotope distribution of the 4charge state of $\mathrm{Au}_{10}(\mathrm{SG})_{10}$.

OPA and TPA spectra calculated using TDDFT approach for the lowest energy catenane structure (see inset Fig. 7.9), are shown in Fig. 7.9. The first excited states of one-photon absorption (OPA) are located between 400 and $300 \mathrm{~nm}$ with increasing intensities are in good agreement with experimental findings. The leading excitations responsible for $S_{1}, S_{2}$ excited states shown also on Fig 7.9 involve Au...Au aurophilic subunits bound to neighbouring sulphur atoms which arise from penetration of the two pentagons into each other. Therefore, although the metal core is not formed due to equal ratio of $\mathrm{Au}-\mathrm{L}$ atoms, aurophilic subunit together with neighbouring sulphur atoms forming two S-Au-S subunits in which the electrons are localized as illustrated by electron localization function (ELF) in Fig. 7.9, 
together with additional small amount of delocalized electrons along Au-S bonds of aurophilic Au...Au subunit are responsible for particular electronic behaviour of $\mathrm{Au}_{10} \mathrm{~L}_{10}$. Due to the fact that $\mathrm{S} 1$ state of OPA is located at $\approx 400 \mathrm{~nm}$, the lowest two photon transition occurs at approximately at $800 \mathrm{~nm}$, with moderate value of cross section which is in agreement with experimentally measured features as shown in Fig 7.9. The leading excitations within S1 state involves $\mathrm{Au}$...Au together with neighbouring sulphur atoms subunit. The calculations of additional TPA states gave rise to S15 located at $\approx 600 \mathrm{~nm}$ with higher TPA cross sections which is not accessible experimentally. However in this case leading excitations involve also MO's with large coefficients at gold atoms within Au...Au subunit. Resonance between OPA and TPA states has not been reached within the manifold of 20 states and the values of transition dipole moments are relatively low.
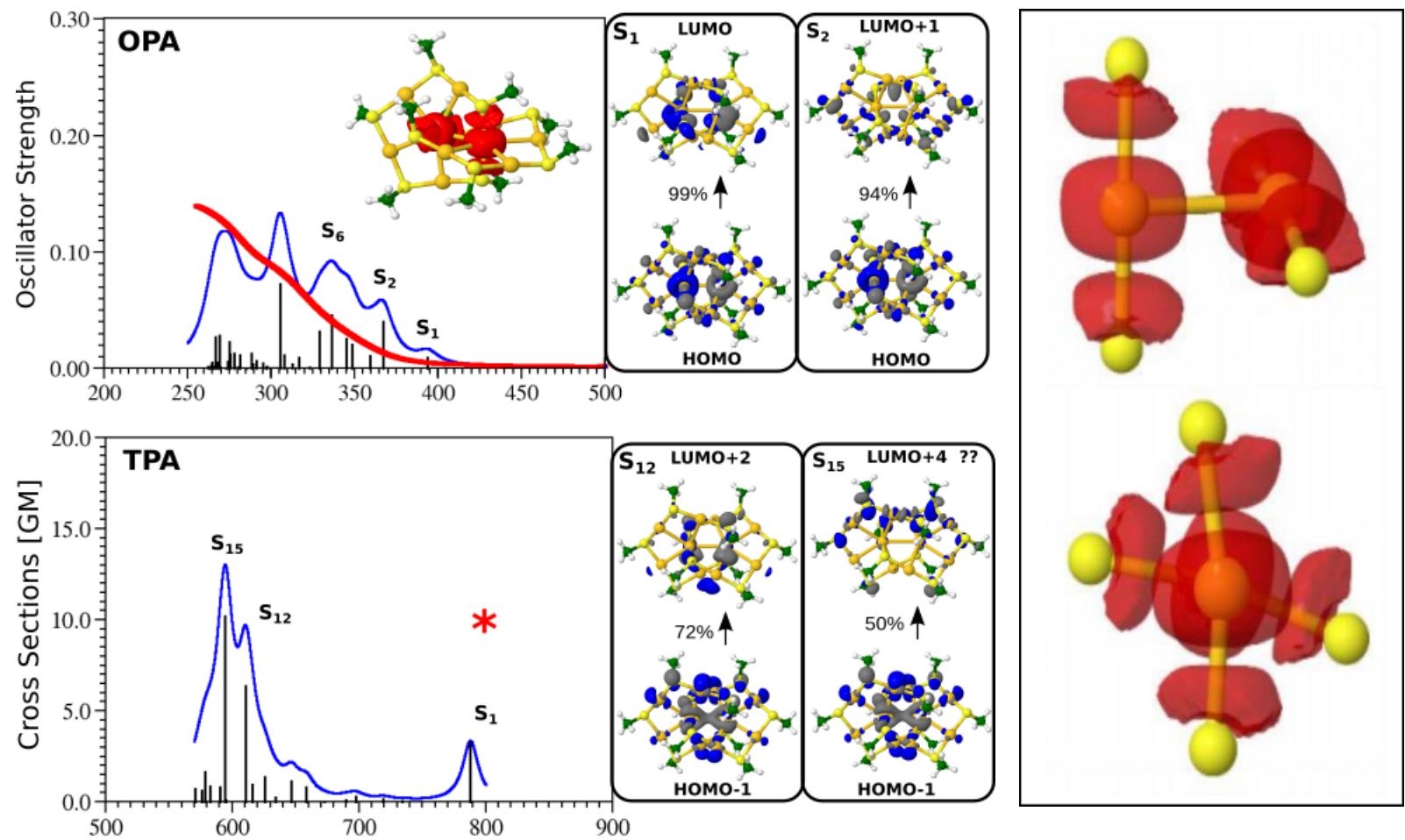

Figure 7.9 : Comparison of TDDFT OPA and TPA spectra for $\mathrm{Au}_{10}(\mathrm{SCH} 3)_{10}$ nanocluster for the lowest energy structure belonging to catenane class with experimental values (red line and star). Structure together with electron localization function (ELF) is shown in left upper part. Leading excitation responsible for the characteristic features of OPA and TPA are shown in the right side. Side and front views of the electron localization function (ELF) of neighbouring Sulphur atoms forming two S-Au-S subunits in which the electrons are localized. Reproduced from SI of J. Phys. Chem. Lett. (2017) DOI: 10.1021/acs.jpclett.7b00611 
One might expect that due to the topology of the catenane structure, a deviation from centrosymmetry should occur leading to large first hyperpolarizabilities. Therefore, the first hyperpolarizability of the as-prepared $\mathrm{Au}_{10}(\mathrm{SG})_{10} \mathrm{NCs}$ was obtained using the hyper-Rayleigh scattering (HRS) technique (described in chapter 6). The first hyperpolarizability $\beta$ determined for an excitation wavelength of $800 \mathrm{~nm}$ for the $\mathrm{Au}_{10}(\mathrm{SG})_{10} \mathrm{NCs}$ is found to be $85(9) \times 10^{-30}$ esu, only slightly lower than the value reported for ligated $\mathrm{Au}_{25}$ clusters, i.e. $128 \times 10^{-30}$ esu. $^{[10]}$ A value of $\sim 125 \times 10^{-30}$ esu is obtained at $785 \mathrm{~nm}$, which is one order of magnitude lower than that of the best SHG dyes used as contrast agents, ${ }^{[23]}$ but in the same range of the hyperpolarizability $\beta$ values reported for gold clusters with chiral ligands as well as silver alloyed gold NCs. ${ }^{[24]}$ The calculated first hyperpolarizability $\beta$ s for the lowest energy isomer with a catenane structure, is in good agreement with the experimental values. ${ }^{[22]}$

As a second example, Knoppe, Häkkinen and Verbiest ${ }^{[25]}$ investigated the static first hyperpolarizabilities $\beta_{0}$ (static hyperpolarizability) based on the experimentally verified structure of the model cluster $\left[\mathrm{Au}_{25}(\mathrm{SH})_{18}\right]^{-}$by means of density-functional theory. The goal of this work was to address the relation between NLO properties and geometric structure of the clusters and also the influence of a chiral ligand on the hyperpolarizability of an otherwise centrosymmetric cluster. An axially chiral 1,1'-binaphthyl-2,2'-dithiol (BINAS) ligand was chosen as a model system. This ligand has been proposed in a number of studies to investigate the influence of chiral ligands on the circular dichroism properties of protected gold clusters. It was shown that BINAS substitutes two monodentate ligands during ligand exchange, connecting two neighboring units via the central sulfur atom of one unit with an outer sulfur atom of the second unit. Based on these findings, a model of $\left[\mathrm{Au}_{25}(\mathrm{SH})_{16}(\mathrm{BINAS}) 1\right]^{-}$was optimized and the calculated hyperpolarizability was reported (see Fig. 7.10). For BINAS 
alone, a value of $4.710^{-30}$ esu was found. On the $\left[\mathrm{Au}_{25}(\mathrm{SH})_{16}(\mathrm{BINAS}) 1\right]^{-}$a value of 44.7 $10^{-30}$ esu was reported (see Fig. 7.10).

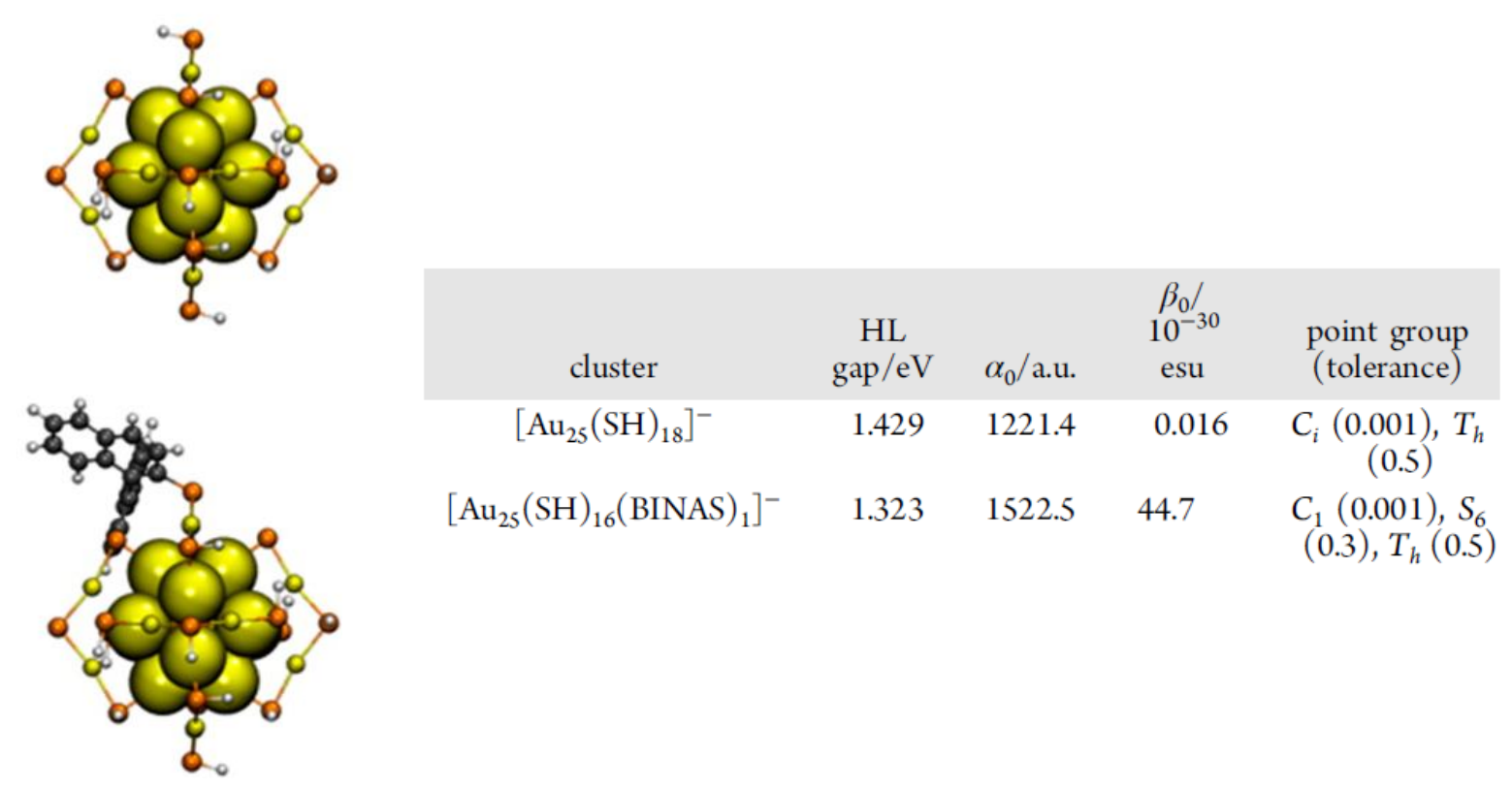

Figure 7.10 : left: Optimized structures of [Au25(SH)18]-, and [Au25(SH)16(BINAS)1]-. All structures are in the same orientation. Au atoms in the icosahedral Au13 core are shown as large spheres. Au, yellow; S, orange; $\mathrm{C}$, gray; $\mathrm{H}, \mathrm{white}$. Bottom: BINAS = 1,1'binaphthyl-2,2'-dithiol. Right : Properties of the $\mathrm{Au}_{25}$ Cluster Substituted with BINAS. Adapted from ref. ${ }^{[25]}$

For $\left[\mathrm{Au}_{25}(\mathrm{SH})_{18}\right]^{-}$, an extremely low value of $0.016 * 10-30$ esu was calculated. Compared to the negligible hyperpolarizability of $\left[\mathrm{Au}_{25}(\mathrm{SH})_{18}\right]^{-}$, this demonstrates the potential of using chiral ligands to induce strong second harmonic response to otherwise centrosymmetric clusters.

Another strategy toward symmetry breaking, proposed by Knoppe et al. ${ }^{[24]}$ was substitution of some of the Au atoms of the cluster by a different metal atoms. For this purpose, they have chosen Ag doping. Single crystal X-ray diffraction (XRD) has shown that Ag atoms substitute $\mathrm{Au}$ atoms of the outer $\mathrm{Au}_{12}$ icosahedron, yielding a $\mathrm{Au} @ \mathrm{Au}_{12-\mathrm{x}} \mathrm{Ag}_{\mathrm{x}}$ core shell structure, for which 82 distinct structures are possible. ${ }^{[26]}$ A significant number of the 82 potential structures of $\left[\mathrm{Au}_{25-x} \mathrm{Ag}_{\mathrm{x}}(\mathrm{SR})_{18}\right]^{-1 / 0}$ will be non-centrosymmetric, and a number of isomers on 
the $\mathrm{x}=4-8$ range is chiral. The $\left[\mathrm{Au}_{25}\left(\mathrm{SCH}_{2} \mathrm{CH}_{2} \mathrm{Ph}\right)_{18}\right]$ cluster does not exhibit any measurable HRS signal (see fig. 7.11). In contrast, HRS measurements of $\mathrm{Au}_{25-x} \mathrm{Ag}_{\mathrm{x}}\left(\mathrm{SCH}_{2} \mathrm{CH}_{2} \mathrm{Ph}\right)_{18}$ yield a first hyperpolarizabilityof $64 \pm 10$. Thus Ag-doping leads to efficient destruction of the centrosymmetry in $\left[\mathrm{Au}_{25}\left(\mathrm{SCH}_{2} \mathrm{CH}_{2} \mathrm{Ph}\right)_{18}\right]^{0}$.
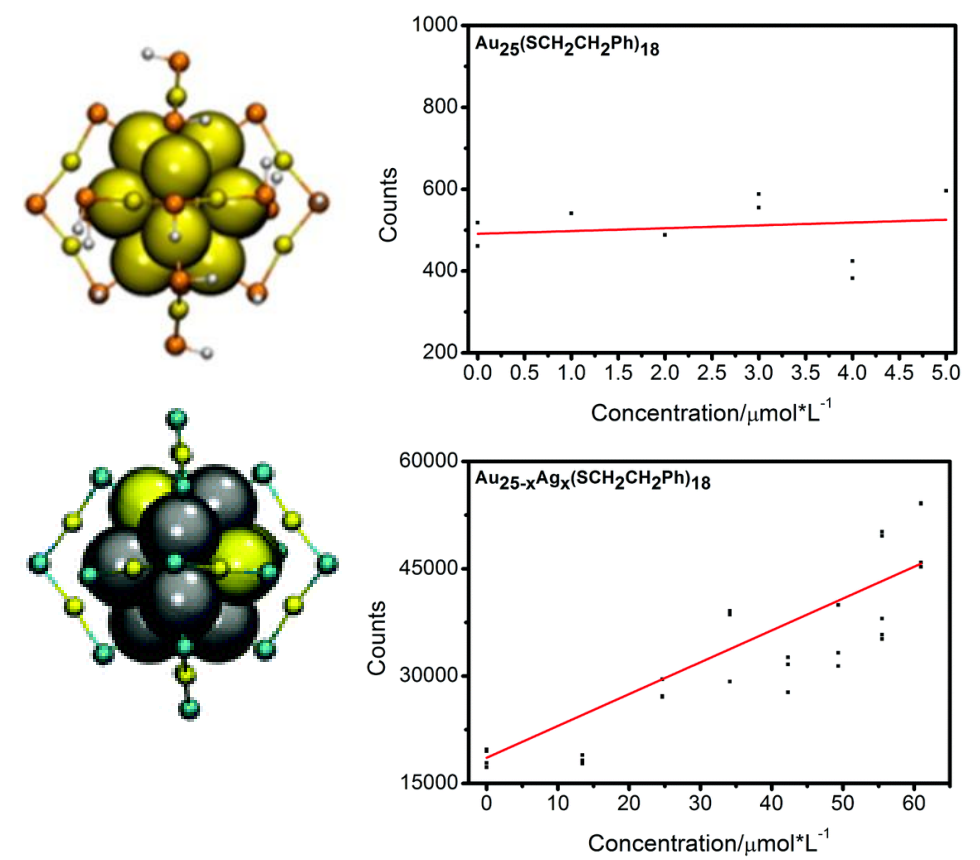

Figure 7.11: (left) The $\mathrm{Au}_{25} \mathrm{~S}_{18}$ and $\mathrm{Au}_{25-x} \mathrm{Ag}_{\mathbf{x}}(\mathrm{S})_{18}$ framework of [Au25(SR)18]- and $\left[\mathrm{Au}_{25-\mathrm{x}} \mathrm{Ag}_{\mathbf{x}}\left(\mathrm{SCH}_{2} \mathrm{CH}_{2} \mathrm{Ph}\right){ }_{18}\right]_{0 .}$. (right) Linear fit of the Hyper-Rayleigh Scattering intensity vs. concentration for $\left[\mathrm{Au}_{25}(\mathrm{SR})_{18}\right]-$ and $\left[\mathrm{Au}_{25-\mathrm{x}} \mathrm{Ag}_{\mathrm{x}}\left(\mathrm{SCH}_{2} \mathrm{CH}_{2} \mathrm{Ph}\right)_{18}\right] 0$. The intensities are corrected for re-absorption at higher concentrations using the Lambert-Beer law. Adapted from ref. ${ }^{24]}$

From these complementary experimental and theoretical investigations, some guidelines for the design of efficient use of thiolate-protected gold clusters can be inferred: At first, as already mentioned in chapter 5, small homo-lumo gaps are desirable. These typically decrease with increasing cluster size; hence, larger clusters may show larger hyperpolarizabilities. Second, the clusters should be inherently chiral. Also, chiral ligands may be used to break the symmetry. The center of inversion in centrosymmetric structures (e.g. $\left[\mathrm{Au}_{25}(\mathrm{SR})_{18}\right]^{-1 / 0}$ ) can also be destroyed by alloying of the cluster with silver. 


\section{References}

[1] X. Qu, Y. Li, L. Li, Y. Wang, J. Liang, J. Liang. Fluorescent Gold Nanoclusters: Synthesis and Recent Biological Application. Journal of Nanomaterials 2015, 2015, 23.

[2] L.-Y. Chen, C.-W. Wang, Z. Yuan, H.-T. Chang. Fluorescent Gold Nanoclusters: Recent Advances in Sensing and Imaging. Anal. Chem. (Washington, DC, U. S.) 2015, 87, 216.

[3] R. Jin, C. Zeng, M. Zhou, Y. Chen. Atomically Precise Colloidal Metal Nanoclusters and Nanoparticles: Fundamentals and Opportunities. Chemical Reviews 2016, 116, 10346.

[4] I. Diez, R. H. A. Ras. Fluorescent silver nanoclusters. Nanoscale 2011, 3, 1963.

[5] R. Jin. Atomically precise metal nanoclusters: stable sizes and optical properties. Nanoscale 2015, 7, 1549.

[6] I. Russier-Antoine, F. Bertorelle, R. Hamouda, D. Rayane, P. Dugourd, Z. Sanader, V. BonacicKoutecky, P.-F. Brevet, R. Antoine. Tuning Ag29 nanocluster light emission from red to blue with one and two-photon excitation. Nanoscale 2016, 8, 2892.

[7] B. Adhikari, A. Banerjee. Facile Synthesis of Water-Soluble Fluorescent Silver Nanoclusters and Hgll Sensing. Chemistry of Materials 2010, 22, 4364.

[8] M. van der Linden, A. Barendregt, A. J. van Bunningen, P. T. K. Chin, D. Thies-Weesie, F. M. F. de Groot, A. Meijerink. Characterisation, degradation and regeneration of luminescent Ag29 clusters in solution. Nanoscale 2016, 8, 19901.

[9] G. Ramakrishna, O. Varnavski, J. Kim, D. Lee, T. Goodson. Quantum-Sized Gold Clusters as Efficient Two-Photon Absorbers. Journal of the American Chemical Society 2008, 130, 5032.

[10] I. Russier-Antoine, F. Bertorelle, M. Vojkovic, D. Rayane, E. Salmon, C. Jonin, P. Dugourd, R. Antoine, P.-F. Brevet. Non-linear optical properties of gold quantum clusters. The smaller the better. Nanoscale 2014, 6, 13572.

[11] S. A. Patel, C. I. Richards, J.-C. Hsiang, R. M. Dickson. Water-Soluble Ag Nanoclusters Exhibit Strong Two-Photon-Induced Fluorescence. Journal of the American Chemical Society 2008, $130,11602$.

[12] C. Xu, W. W. Webb. Measurement of two-photon excitation cross sections of molecular fluorophores with data from 690 to $1050 \mathrm{~nm}$. JOSA B 1996, 13, 481.

[13] Z. Wu, R. Jin. On the Ligand's Role in the Fluorescence of Gold Nanoclusters. Nano Letters 2010, 10, 2568.

[14] P. C. Ray. Size and Shape Dependent Second Order Nonlinear Optical Properties of Nanomaterials and Their Application in Biological and Chemical Sensing. Chemical Reviews 2010, 110, 5332.

[15] J. Nappa, G. Revillod, I. Russier-Antoine, E. Benichou, C. Jonin, P. F. Brevet. Electric dipole origin of the second harmonic generation of small metallic particles. Physical Review B 2005, $71,165407$.

[16] J. Nappa, I. Russier-Antoine, E. Benichou, C. Jonin, P. F. Brevet. Second harmonic generation from small gold metallic particles: From the dipolar to the quadrupolar response. The Journal of Chemical Physics 2006, 125, 184712.

[17] I. Russier-Antoine, E. Benichou, G. Bachelier, C. Jonin, P. F. Brevet. Multipolar Contributions of the Second Harmonic Generation from Silver and Gold Nanoparticles. The Journal of Physical Chemistry C 2007, 111, 9044.

[18] J. Duboisset, A. Deniset-Besseau, E. Benichou, I. Russier-Antoine, N. Lascoux, C. Jonin, F. Hache, M.-C. Schanne-Klein, P.-F. Brevet. A Bottom-Up Approach to Build the 
Hyperpolarizability of Peptides and Proteins from their Amino Acids. The Journal of Physical Chemistry B 2013, 117, 9877.

[19] A. Tlahuice-Flores, M. Jose-Yacaman, R. L. Whetten. On the structure of the thiolated Au15 cluster. Physical Chemistry Chemical Physics 2013, 15, 19557.

[20] D.-e. Jiang, S. H. Overbury, S. Dai. Structure of Au15(SR)13 and Its Implication for the Origin of the Nucleus in Thiolated Gold Nanoclusters. Journal of the American Chemical Society 2013, 135, 8786.

[21] M. Zhu, C. M. Aikens, F. J. Hollander, G. C. Schatz, R. Jin. Correlating the Crystal Structure of A Thiol-Protected Au25 Cluster and Optical Properties. Journal of the American Chemical Society 2008, 130, 5883.

[22] F. Bertorelle, I. Russier-Antoine, N. Calin, C. Comby-Zerbino, A. Bensalah-Ledoux, S. Guy, P. Dugourd, P.-F. Brevet, Ž. Sanader, M. Krstić, V. Bonačić-Koutecký, R. Antoine. Au10(SG)10: A Chiral Gold Catenane Nanocluster with Zero Confined Electrons. Optical Properties and FirstPrinciples Theoretical Analysis. The Journal of Physical Chemistry Letters 2017, 1979.

[23] P. J. Campagnola, L. M. Loew. Second-harmonic imaging microscopy for visualizing biomolecular arrays in cells, tissues and organisms. Nature Biotechnology 2003, 21, 1356.

[24] N. Van Steerteghem, S. Van Cleuvenbergen, S. Deckers, C. Kumara, A. Dass, H. Hakkinen, K. Clays, T. Verbiest, S. Knoppe. Symmetry breaking in ligand-protected gold clusters probed by nonlinear optics. Nanoscale 2016, 8, 12123.

[25] S. Knoppe, H. Häkkinen, T. Verbiest. Nonlinear Optical Properties of Thiolate-Protected Gold Clusters: A Theoretical Survey of the First Hyperpolarizabilities. The Journal of Physical Chemistry C 2015, 119, 27676.

[26] S. Knoppe. Generation of isomers for icosahedral clusters $A 12-x B x(x=0-12)$ from a symmetry-based algorithm. Polyhedron 2015, 100, 351. 


\section{Chapter 8}

\section{Concluding remarks and Outlook}

In this book we have demonstrated that liganded gold and silver quantum clusters can be viewed as a new class of nonlinear optical materials. Recent studies have clearly demonstrated that ligated noble (silver $(\mathrm{Ag})$, gold $(\mathrm{Au}), \ldots)$ metal NCs, with few to hundred atoms exhibit remarkable nonlinearities. ${ }^{[1][2]}$ Thus, these ligated nanoclusters may provide a valuable route within the nonlinear regime. We have described in our joint experimental and theoretical investigations how to improve TPA properties in such nanoclusters, through the concept of "Ligand-Core" NLO-phores. ${ }^{[2]}$ The structures of metallic core, its charge and symmetry (influencing the SHG and TPEF cross-sections) and the ligands stabilizing the core, play major role in TPA efficiencies. Two aspects will determine the future of nonlinear materials based on "ligand-core" NLO-phores concept: i) theoretical design of hybrid systems with significant nonlinearities based on development of rules according to which these properties can be achieved providing guidelines to experiments; ii) preparation of amount of 
materials sufficient to be used for applications. A large exploration of size and nature of ligands will allow to further probe the influence of the core-ligand interface in NLO efficiencies of thiol-liganded metalic NCs. However, the main conclusion up to now is that liganded silver and gold quantum clusters are excellent two-photon absorbers but present limited two-photon emission cross sections.

Concerning non-linear emissive properties, progresses both experimentally and theoretically are needed to bridge the gap between large TPA and large TPEF cross sections. It is wellknown that the rigidity of the ligand shell may affect both linear and nonlinear emissive properties of AuNCs. A simple way to "rigidify" the protective shell is to use proteins instead of thiolated molecules. Xie et al. ${ }^{[3]}$ have developed a facile, one-pot, "green" synthetic technique based on a common commercially available protein, bovine serum albumin (BSA). The as-prepared AuNCs were stable at the physiological temperature and $\mathrm{pH}$ with a quantum yield (QY) 6\%. The as-synthesized BSA-AuNCs have 25 gold atoms in the core, exhibit red emission (maximum of emission wavelength located at $640 \mathrm{~nm}$ ), excellent stability, good biocompatibility and easy surface modification. This route could be explored with different size of protein templates, in order to "tune" the number of atoms in the core thanks to the size of the protein. Using (bio)organic thiolate templates, further enhancement can be achieved by rigidifying the metal shell. Pyo et al. ${ }^{[4]}$ have shown that it is possible to achieve luminescence quantum yield $>60 \%$ by rigidifying the gold shell by lowering the medium temperature or binding with bulky groups (tetraoctylammonium (TOA) cations).

SHG is a coherent phenomenon involving radiative scattering asymmetry sensitivity (interface probe) while TPEF is a volume incoherent phenomenon involving radiative absorption and re-emission (volume probe). In particular, SHG and TPEF have gained overwhelming popularity in the biology community to enhance contrasts and improve resolution. ${ }^{[5]}$ The combination of the two processes intrinsically provides different contrasts 
(see Figure 8.1, left). For instance SHG and TPFE microscopies invoked a great interest to study biological processes and structural information in cellulo. ${ }^{[6]}$ By controlling the nanoclusters size, rigidity and/or asymmetry (see Figure 8.1, right), we will provide the highest corresponding SHG and TPEF cross-sections. Possible routes allowing to enhance SHG efficiency would consist in playing with composition (by doping metal core with silver, palladium, or platinum) and/or asymmetry (both in the metal core and the ligand shell organization).
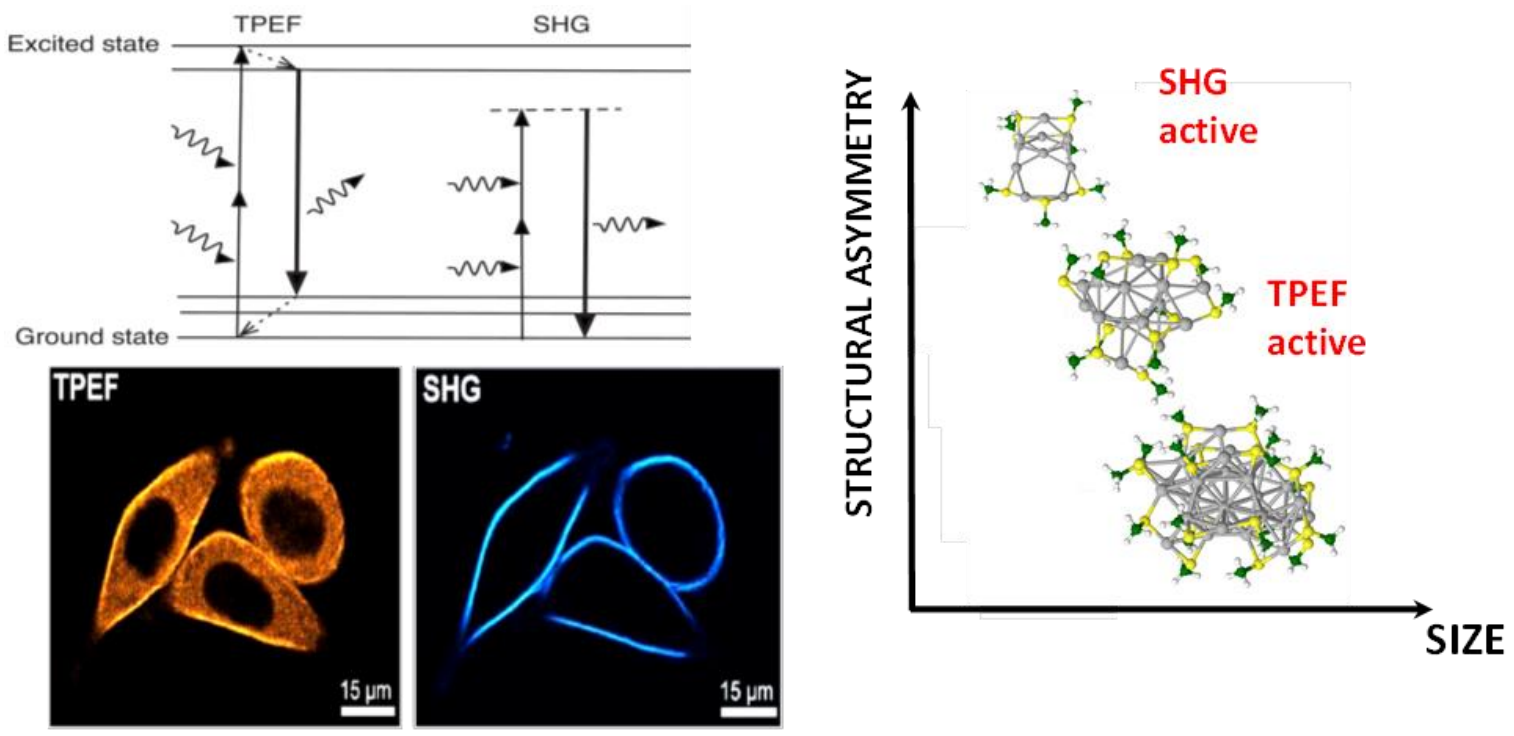

Figure 8.1 : (left) Schematic representation of nonlinear processes: TPA followed by TPEF and SHG. Conceptual view of multiphoton optics with complementary information gained with SHG (surface) and TPEF (volume) in cells. (adapted from Ref.[3]) (right) NLO properties of metal clusters as a function of size and of asymmetry $\left(\mathrm{Ag}_{11}, \mathrm{Ag}_{15}\right.$, and $\mathrm{Ag}_{31}$ are shown as example).

A final important aspect is the ability to provide simple synthesis aiming to produce large scale amount of hybrid metallic-thiolate liganded clusters using new concepts are highly desirable. The concept of "size specific" for nanoclusters and targeted the one-pot-for-onesize synthesis of nanoclusters without the need for electrophoretic or chromatographic isolation of size mixed products was introduced in late 2000, by Rongjao Jin et al.. These 
findings opened the route for synthesis of ligated AuNCs. ${ }^{[7]}$ The control of the nanocluster size remains a huge challenge to chemists. Therefore, the nanoclusters with sizes belonging to the non-scalabe regime need to be fully explored, using different preparative approaches, in order to produce scalable and precise synthesis of AuNCs with different metal cores and different ligands. This approach consists in reducing the gold ions into zero valent atoms, and then AuNCs are formed with the nucleation of the $\mathrm{Au}$ atoms. More precisely, gold salts $\left[\mathrm{AuCl}_{4}\right]^{-}$are dissolved in water; the thiols are added to the mixture to reduce $\mathrm{Au}^{3+}$ ions into $\mathrm{Au}^{+}$ions and to form $\mathrm{Au}^{+}-\mathrm{SR}$ complexes or polymers; then the $\mathrm{Au}^{+}$polymers are reduced by adding the reducing agent into thiolate-protected AuNCs. We have recently reported a simple synthesis of hybrid metal:thiolated ligand (M:L) clusters using a new concept based on the interaction of an organic base with the ligand molecules providing a steric hindrance to the formation of gold-thiolate complexes at the early stage of synthesis that leads to aqueous solutions containing at the end well-defined $\mathrm{Au}_{15} \mathrm{~L}_{13}$ clusters.

Finally the nature of the ligand is important. Glutathione (GSH), a ubiquitous low-molecular weight thiol, played a significant role in making AuNCs which showed good water solubility, bioactive surface, and high stability. AuNCs can be prepared with glutathione and several other ligands such as cysteine and its derivatives, and tiopronin, thiolate $\beta$-cyclodextrin, mercaptopropionic acid, bidentate dihydrolipoic acid (DHLA) and penicillamine. These ligands could be used to produce thiol-liganded AuNCs and to further probe the influence of the core-ligand interface in NLO efficiencies. Also highly conjugated thiol ligands $\left(\mathrm{HSPhNH}_{2}\right)$ could be explored to probe the influence of ligand electrons on NLO efficiencies.

Beyond multiphoton bioimaging applications, our findings may find some outcome in other fields. As mentioned, liganded metal quantum clusters present a huge two-photon absorption cross section but are moderate two-photon excited emitters... This drawback for using probes as contrast agents for multiphoton microscopy might be used for cancer therapy. Large TPA 
means that the particles are efficient light absorbers. The absorbed photon energy (light) can be converted into strong localized heat, which may destroy cancer cells via hyperthermia. This process can be viewed as a two-photon photothermal therapy and needs to be compared with the more usual plasmonic photothermal therapy (PPTT). ${ }^{[8,9]}$ Pilot photothermal ablation experiments were already performed with gold nanourchins (AuNUs). In figure 8.2, due to strong absorption of the NIR femtosecond laser light of AuNUs within 3D-glioblastoma (GBM) cultures, a localized bubble is formed. The efficiency of inducing cell death needs to be evaluated in the future with size specific gold and silver clusters.
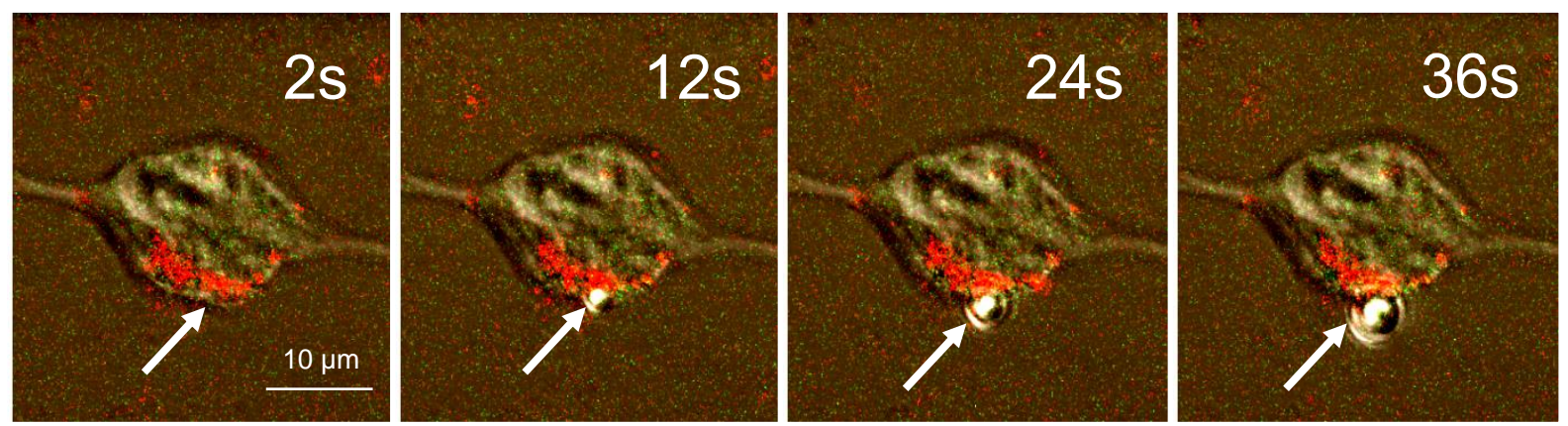

Figure 8.22 : Bubble formation in human GBM cell with internalized gold nanourchins upon irradiation of $750 \mathrm{~nm}$ Ti:sapphire femtosecond laser (unpublished results, by courtesy of Dusica Maysinger, McGill University, Montreal, Canada).

In contrast to the omnifarious functionalization techniques for successfully handling the plasmonic AuNPs, the surface chemical engineering of fluorescent AuNCs presents a daunting challenge and is still in its infancy. The major reason is their higher surface to volume ratio and the extraordinary sensitivity of the fluorescence response to the surface ligand and relevant environment. Therefore, it is necessary to prepare fluorescent AuNCs which can be easily functionalized through random and irregular ligand modification by using specific functionalized molecules.

For bio-applications, biotin conjugated to AuNCs will efficiently target streptavidin proteins. Our preliminary investigations showed that biotins can be efficiently grafted to $\mathrm{Au}_{15} \mathrm{NCs}$ (see 
Figure 8.3). We also managed to use hydrazine to produce functional AuNCs-hydrazides that can react smoothly and nearly quantitatively with various carbonyl groups encountered in biomolecules (proteins subjected to oxidative stress, glycosylated proteins pre-activated by periodate oxidation including antobodies, and oligonucleotides with aldehyde moieties). Bioconjugation with the cyclic-RGD peptide was shown to enhance tumor targeting by severalfold, over non-RGD conjugated ones. This targeting by simply coupling the surface carboxylic groups of AuNCs to RGD could be also explored.

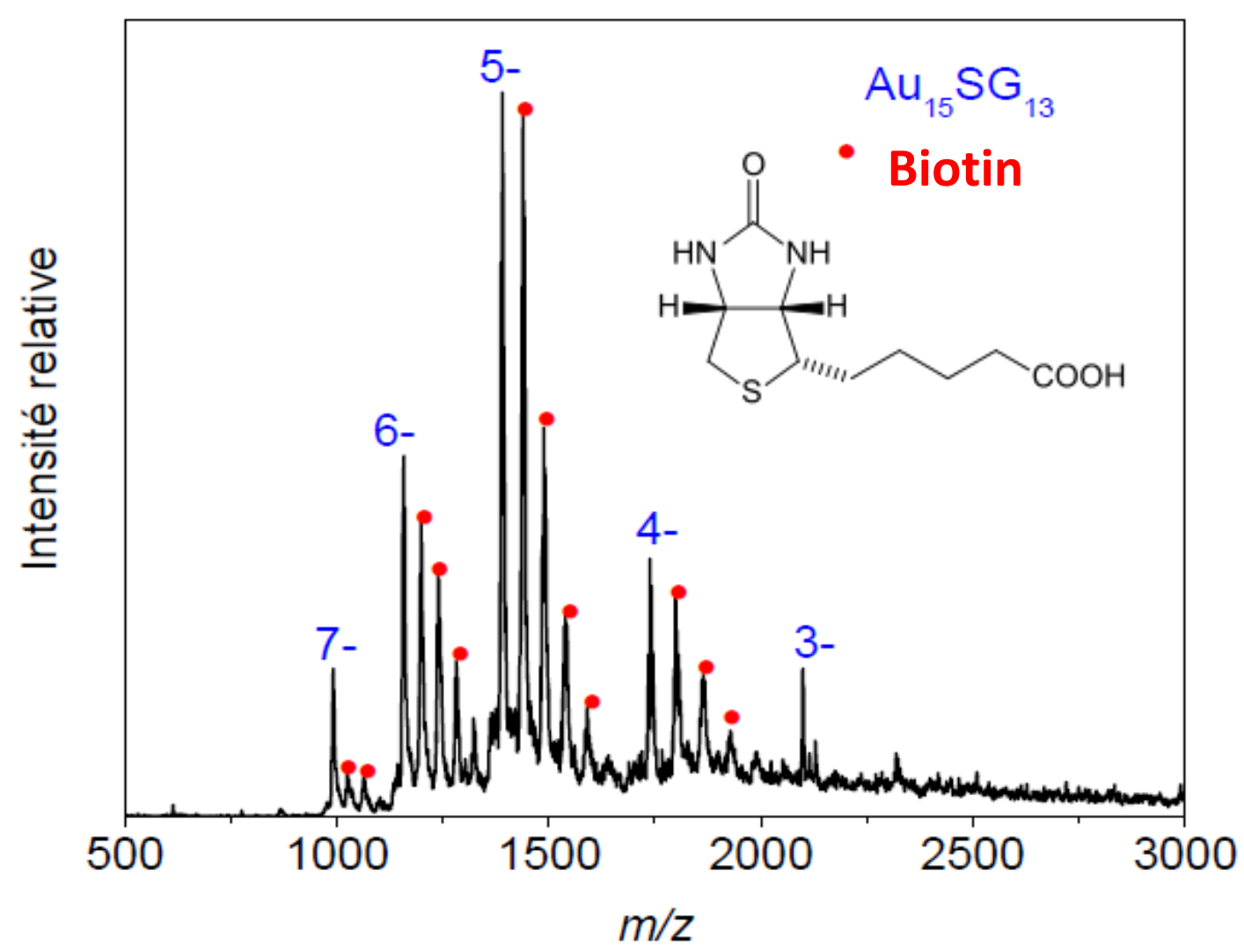

Figure 3 : ESI mass spectrum of biotin- $\mathrm{Au}_{15}$ protected clusters acquired under gentle ESI conditions. A charge state distribution was observed ranging from $[\mathrm{M}-3 \mathrm{H}]^{3-}$ to $[\mathrm{M}-7 \mathrm{H}]^{7^{-}}$. Biotin molecules (chemical structure in inset) are covalently attached to the surface glutathione (SG) ligands. 
Gold nanoclusters are extensively studied in solution for their distinctive optical properties. However, many applications of nanoclusters involve their use in the solid state like films. $\mathrm{Au}_{25} \mathrm{NCs}$ in polystyrene hosts was used as the model for studying the optical properties of nanocluster films (see figure 8.4). It was found by Goodson and co-workers ${ }^{[10]}$ that the formation of nanocluster films enormously reduces the cluster-cluster distances to a few nanometers. An emission enhancement was observed and was attributed to the energy transfer between clusters due to the small cluster-cluster distance. Two-photon Z-scan measurements provide huge two-photon absorption cross sections $\left(10^{6} \mathrm{GM}\right)$, that is one order of magnitude higher than it is in solution. This strong enhancement suggests a strong dipole coupling between clusters. Metal nanoclusters embedded in polymeric films are a new way to produce nanoclusters in the solid state with enhanced linear and nonlinear optical properties.
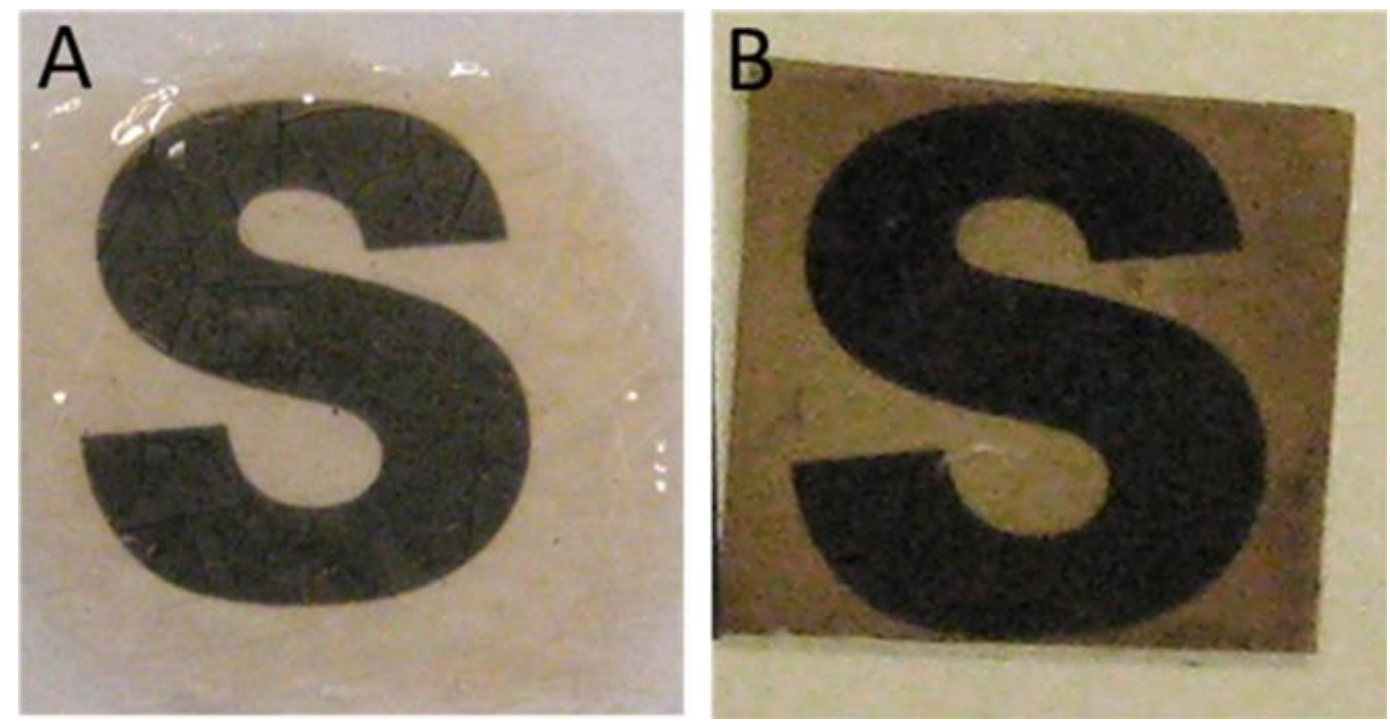

Figure 8.4: Samples of Au25(GSH)18/PVP films (A) and Au25(C6S)18/PS films (B) at different nanoclusters loading. Adapted from ref. ${ }^{[10]}$ 
References

[1] S. H. Yau, O. Varnavski, T. Goodson. An Ultrafast Look at Au Nanoclusters. Accounts of Chemical Research 2013, 46, 1506.

[2] I. Russier-Antoine, F. Bertorelle, N. Calin, Z. Sanader, M. Krstic, C. Comby-Zerbino, P. Dugourd, P.-F. Brevet, V. Bonacic-Koutecky, R. Antoine. Ligand-core NLO-phores: a combined experimental and theoretical approach to the two-photon absorption and two-photon excited emission properties of small-ligated silver nanoclusters. Nanoscale 2017, 9, 1221.

[3] J. Xie, Y. Zheng, J. Y. Ying. Protein-Directed Synthesis of Highly Fluorescent Gold Nanoclusters. Journal of the American Chemical Society 2009, 131, 888.

[4] K. Pyo, V. D. Thanthirige, K. Kwak, P. Pandurangan, G. Ramakrishna, D. Lee. Ultrabright Luminescence from Gold Nanoclusters: Rigidifying the $\mathrm{Au}(\mathrm{I})$-Thiolate Shell. Journal of the American Chemical Society 2015, 137, 8244.

[5] L. Moreaux, O. Sandre, S. Charpak, M. Blanchard-Desce, J. Mertz. Coherent Scattering in Multi-Harmonic Light Microscopy. Biophysical Journal, 80, 1568.

[6] E. E. Hoover, J. A. Squier. Advances in multiphoton microscopy technology. Nat Photon 2013, 7, 93.

[7] M. Zhu, C. M. Aikens, F. J. Hollander, G. C. Schatz, R. Jin. Correlating the Crystal Structure of A Thiol-Protected Au25 Cluster and Optical Properties. Journal of the American Chemical Society 2008, 130, 5883.

[8] E. Hutter, D. Maysinger. Gold-nanoparticle-based biosensors for detection of enzyme activity. Trends in Pharmacological Sciences, 34, 497.

[9] E. Hutter, D. Maysinger. Gold nanoparticles and quantum dots for bioimaging. Microscopy Research and Technique 2011, 74, 592.

[10] R. Ho-Wu, S. H. Yau, T. Goodson. Linear and Nonlinear Optical Properties of MonolayerProtected Gold Nanocluster Films. ACS Nano 2016, 10, 562. 